University of Louisville

ThinkIR: The University of Louisville's Institutional Repository

Electronic Theses and Dissertations

$11-2007$

\title{
A voice operated musical instrument.
}

Travis R. Gault

University of Louisville

Follow this and additional works at: https://ir.library.louisville.edu/etd

\section{Recommended Citation}

Gault, Travis R., "A voice operated musical instrument." (2007). Electronic Theses and Dissertations. Paper 482.

https://doi.org/10.18297/etd/482

This Master's Thesis is brought to you for free and open access by ThinkIR: The University of Louisville's Institutional Repository. It has been accepted for inclusion in Electronic Theses and Dissertations by an authorized administrator of ThinkIR: The University of Louisville's Institutional Repository. This title appears here courtesy of the author, who has retained all other copyrights. For more information, please contact thinkir@louisville.edu. 


\title{
A Voice Operated Musical Instrument
}

By

Travis R. Gault

B.S. ECE, University of Louisville, 2004 (magna cum laude)

B.S. CECS, University of Louisville, 2006 (magna cum laude)

\author{
A Thesis \\ Submitted to the Faculty of the \\ University of Louisville \\ Speed School of Engineering \\ in Partial Fulfillment of the Requirements \\ for the Professional Degree of
}

\section{MASTER OF ENGINEERING}

\author{
Department of Electrical and Computer Engineering \\ University of Louisville \\ Louisville, Kentucky
}

November 28, 2007 


\title{
A Voice Operated Musical Instrument
}

\author{
By \\ Travis R. Gault \\ B.S. ECE, University of Louisville, 2004 \\ B.S. CECS, University of Louisville, 2006
}

A Thesis Approved on

November 28, 2007

By the following Thesis Committee:

Dr. Thomas Cleaver, Thesis Director

Dr. Barry Horowitz

Dr. Ahmed Desoky 


\section{DEDICATION}

To my friends, both students and faculty, whose support has been tremendous throughout my college career, especially to my parents who have supported me since day zero, and who have been wholly committed to the belief that I may actually finish school someday and "get a J-O-B". 


\section{ACKNOWLEDGEMENTS}

I would like to thank all the mentors that have given so much over most of the past

decade, namely the faculty and staff at Speed School. I would also like to extend a special gratitude toward my Thesis Director (Dr. Cleaver) and Thesis Committee (Drs. Horowitz and Desoky) and Tom Carroll in the Electronics Shop for taking extra time from their days to assist and guide me on this challenging path. Thank you. 


\begin{abstract}
Many mathematical formulas and algorithms exist to identify pitches formed by human voices, and this has continued to be popular in the fields of music and signal processing. Other systems and research perform real time pitch identification implemented by using PCs with system clocks faster than $400 \mathrm{MHz}$. This thesis explores developing an embedded RPTI system using the average magnitude difference function (AMDF), which will also use MIDI commands to control a synthesizer to track the pitch in near real time.

The AMDF algorithm was simulated and its performance analyzed in MATLAB with pre-recorded sound files from a PC. Errors inherent to the AMDF and the hardware constraints led to noticeable pitch errors. The MATLAB code was optimized and its performance verified for the Motorola 68000 assembly language. This stage of development led to realization that the original design would have to change for the processing time required for the AMDF implementation. Hardware was constructed to support an $8 \mathrm{MHz}$ Motorola 68000, analog input, and MIDI communications. The various modules were constructed using Vectorbord(C prototyping board with soldered tracks, wires and sockets. Modules were tested individually and as a whole unit. A design flaw was noticed with the final design, which caused the unit to fail during program execution while operating in a stand-alone mode.

This design is a proof of concept for a product that can be improved upon with newer components, more advanced algorithms and hardware construction, and a more aesthetically pleasing package. Ultimately, hardware limitations imposed by the available equipment in addition to a hidden design flaw contributed to the failure of this stand-alone prototype.
\end{abstract}




\section{TABLE OF CONTENTS}

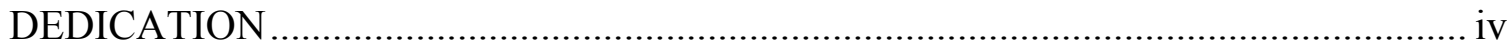

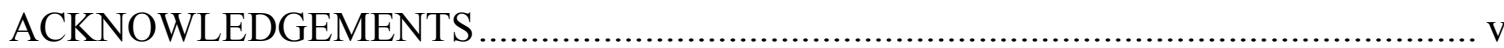

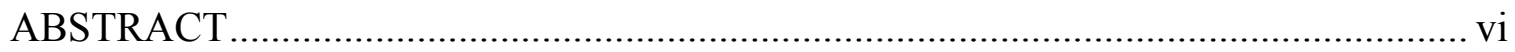

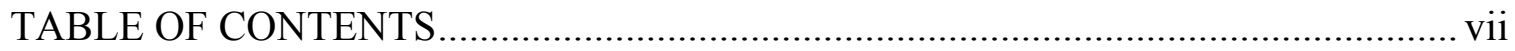

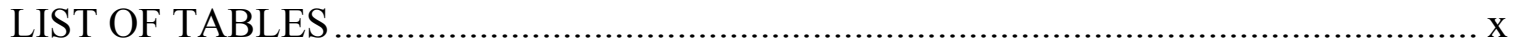

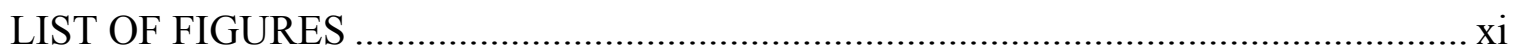

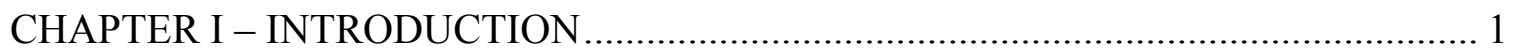

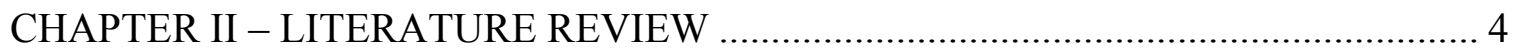

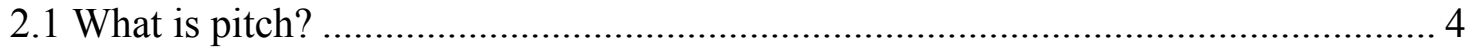

2.2 The human vocal range and the ideal choice of sampling rate ............................... 5

2.3 Frequency Identification (Pitch-Tracking) Techniques .............................................. 6

2.3.1 Problems Associated with Pitch Detection of Vocal Signals ............................ 7

2.3.2 Counting Zero-Crossings ................................................................................ 9

2.3.3 Frequency Domain Analysis using the Discrete Fourier Transform (DFT) .... 10

2.3.4 Auto-Correlation Function........................................................................ 12

2.3.5 Average Magnitude-Difference Function (AMDF)........................................ 13

2.3.6 Some Other Techniques Worth Mentioning .................................................... 14

2.3.7 Anticipated Problems with the AMDF …………........................................ 17

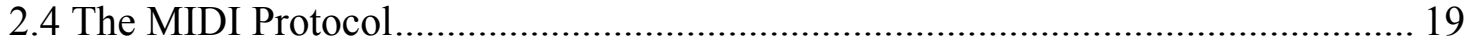

2.4.1 Message Format ................................................................................. 19

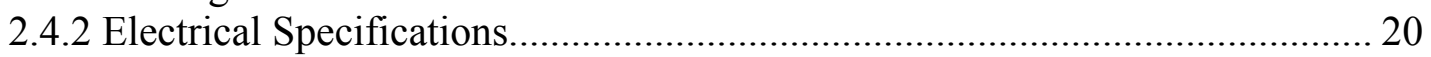

2.4.3 Timing Considerations ........................................................................ 21

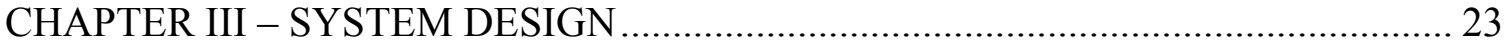

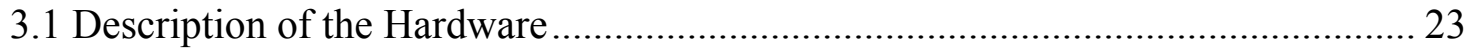

3.1.1 M68000 Connections ............................................................................... 24

3.1.2 The Memory Management Unit (MMU) ..................................................... 25

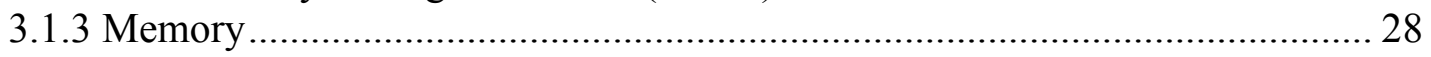

3.1.4 Data Transmission Acknowledge (DTACK') Signal ..................................... 29

3.1.5 Valid Peripheral Address (VPA') Signal..................................................... 30

3.1.6 Bar Graph LED Package and Output Data Register (74LS273)...................... 31

3.1.7 Serial Communications Interface (SCI) for Terminal Communications .......... 32

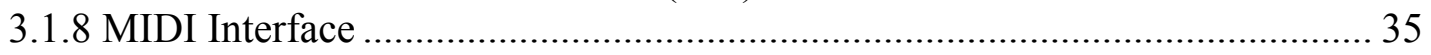

3.1.9 Microphone Input Amplification and Filtering................................................. 36

3.1.10 A/D Converter (ADC0809) and Sampling Frequency Generator................... 39

3.1.11 A/D Interrupt Acknowledgement (IACK') and MMU Reset (MRST') ........ 42

3.1.12 Power-On Reset Circuit ......................................................................... 44

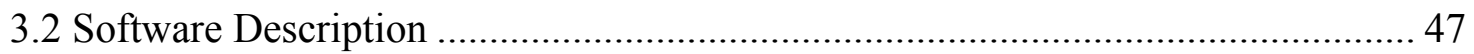

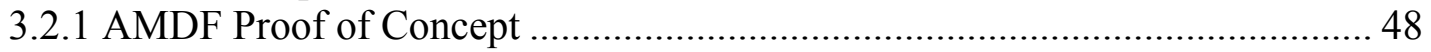

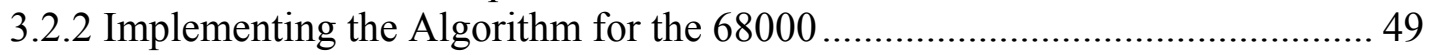

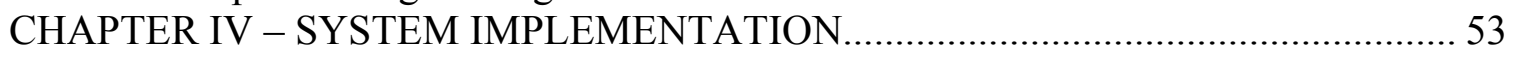

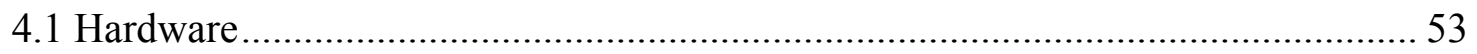

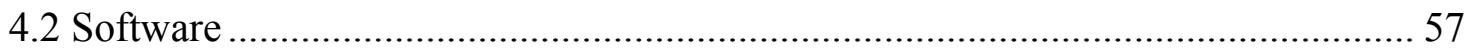

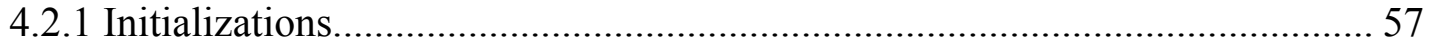

4.2.2 Sampling and performing the AMDF (ADCIRQ) ......................................... 58 


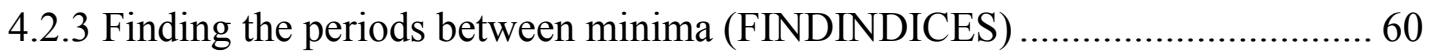

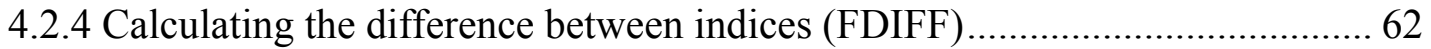

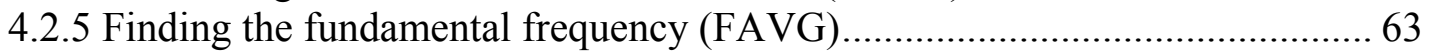

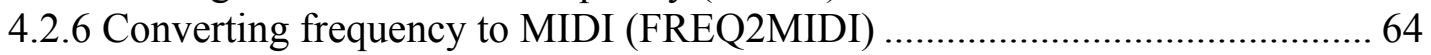

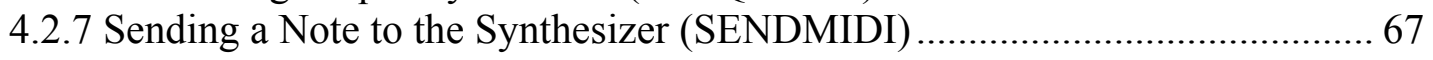

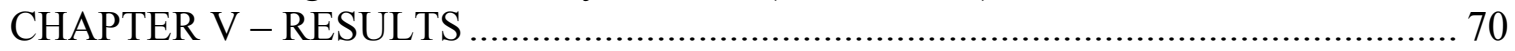

5.1 MATLAB AMDF Proof of Concept Simulations …........................................... 70

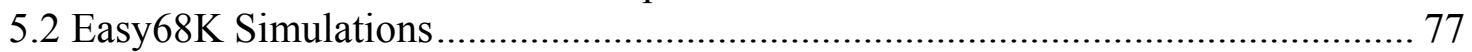

5.3 Hardware Debugging with the Deneb Emulator................................................. 78

5.3.1 Checking Physical Connections............................................................... 79

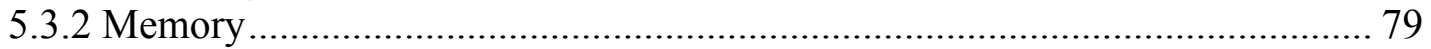

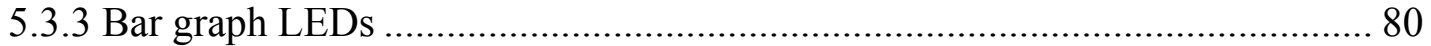

5.3.4 A/D converter................................................................................... 81

5.3.5 Power-on reset circuitry, serial communications interface (SCI), and sampling

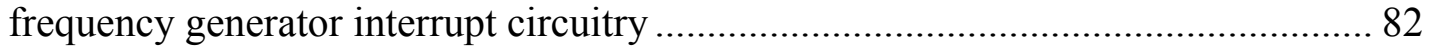

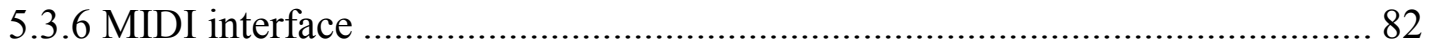

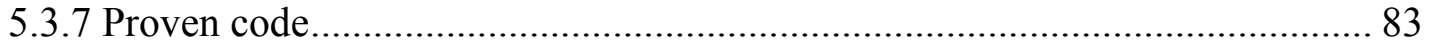

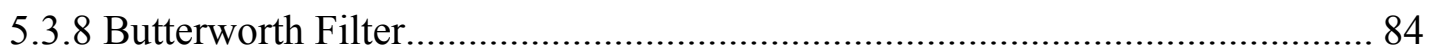

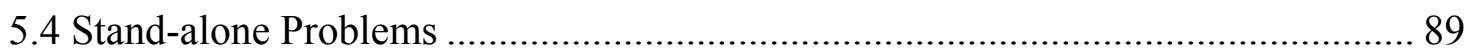

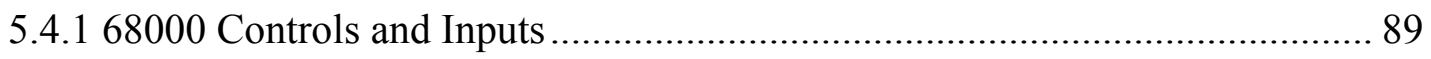

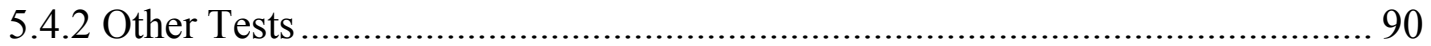

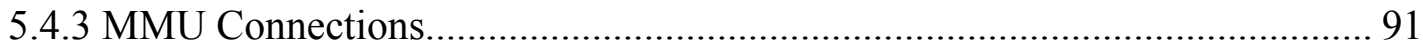

5.4.4 Checking the Memory Space ................................................................... 92

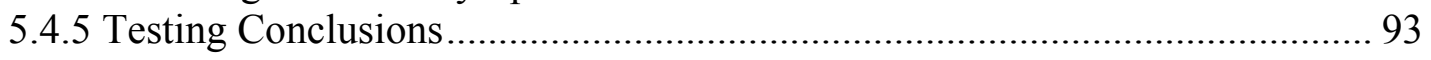

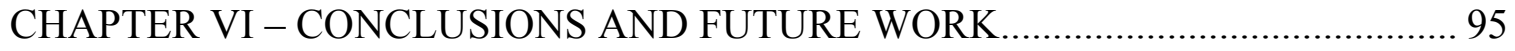

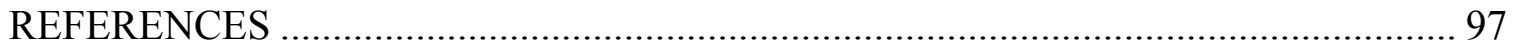

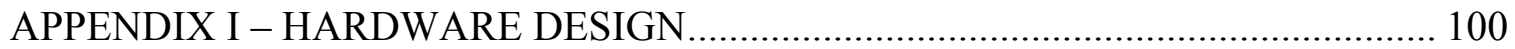

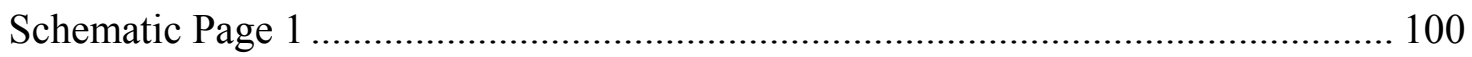

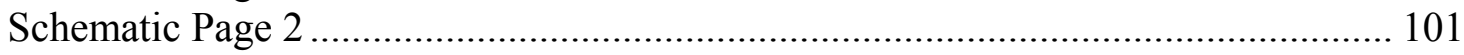

Board Layout - Top View of All Components...................................................... 102

Board Layout - Top View of Top Components .................................................... 103

Board Layout - Bottom View of Bottom Components ........................................... 104

APPENDIX II - SIMULATION SOURCE CODE................................................... 105

Butterworth Filter Spice Simulation File for Ideal Component Values (BWLPF.spc)

Butterworth Filter Spice Simulation File for Actual Component Values (BWLPF-

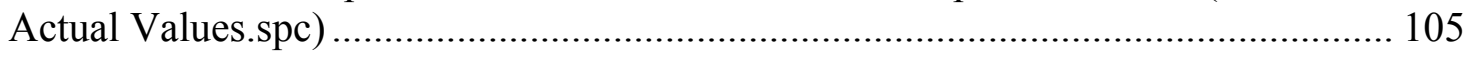

Butterworth Filter Spice Simulation File for Actual Component Values with Updated

Inductance and Resistance Values (BWLPF-Actual Values with LR.spc) ............... 105

Butterworth Filter Spice Simulation File for Actual Component Values with New Inductance and Resistance Values (BWLPF-Actual Values with new LR.spc)........ 106

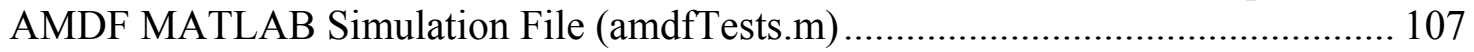

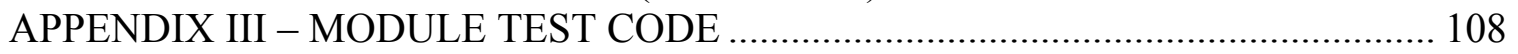

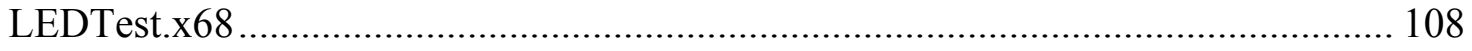

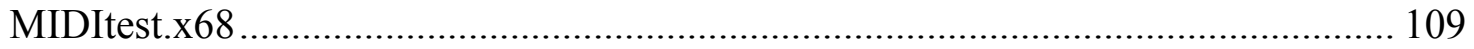

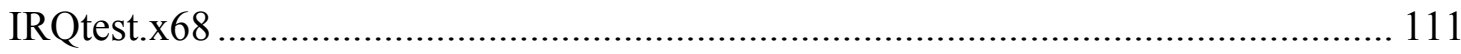




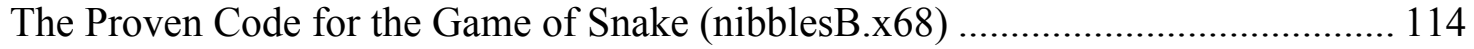

APPENDIX IV - DESIGN SOURCE CODE ........................................................ 126

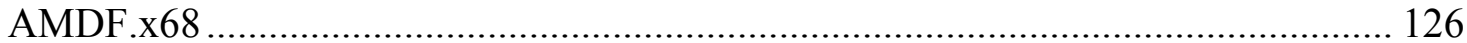




\section{LIST OF TABLES}

Table 2.1: Computational considerations for the seven pitch detectors on the Nova 800 minicomputer. Taken from [Rabiner 1976] ....................................................... 16

Table 2.2: Number of gross pitch errors - unsmoothed. Taken from [Rabiner 1976]. .. 17

Table 2.3: Performance scores based on sum of gross pitch errors - unsmoothed. Taken

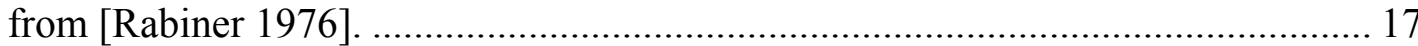

Table 2.4: MIDI commands................................................................................. 20

Table 3.1: Exception vector assignments. Taken from [Motorola 1993] ...................... 43

Table 4.1: Board layout color-coding conventions................................................... 56

Table 4.2: Pitches corresponding to actual fundamental frequencies, valid integer representations from the AMDF, linked list memory offsets, and MIDI note

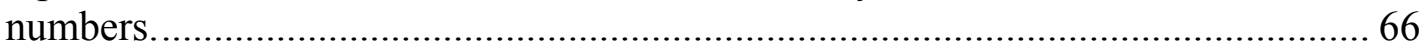

Table 5.1: AMDF simulation results from MATLAB for F3 ................................... 74

Table 5.2: AMDF simulation results from MATLAB for A3 .................................... 75

Table 5.3: AMDF simulation results from MATLAB for C4. FFT errors are shown in

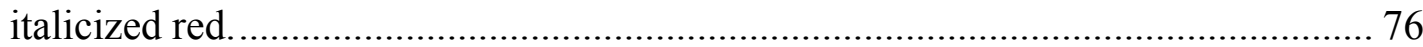




\section{LIST OF FIGURES}

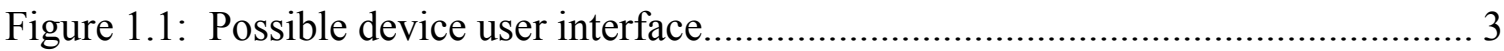

Figure 2.1: Vocal ranges for singers. Taken from [Yale 2005] .................................... 6

Figure 2.2: Two waveform measurements which can be used to define pitch markers.

Taken from [Rabiner 1976] ......................................................................... 8

Figure 2.3: Perfect sinusoid (a) and vocal sample (b) at the same frequency. ................. 9

Figure 2.4: Power spectrum of the vocal sample from Figure 2.3b.............................. 11

Figure 2.5: Computer Sound Card Game Port to Standard MIDI Connector [MMA

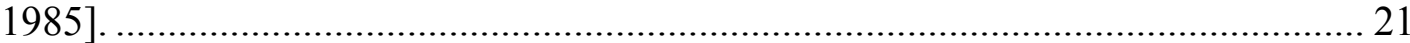

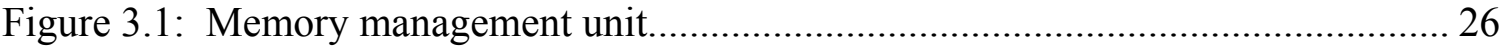

Figure 3.2: (a) Even NVRAM and (b) Odd NVRAM and connections. ......................... 28

Figure 3.3: Data transfer acknowledge/valid peripheral address circuitry..................... 29

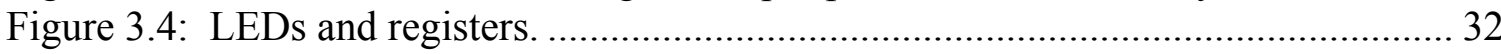

Figure 3.5: SCI asynchronous communications interface adapter.................................. 33

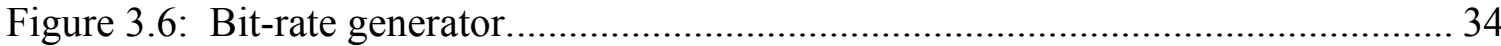

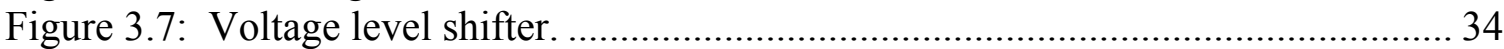

Figure 3.8: MIDI asynchronous communications interface adapter............................... 35

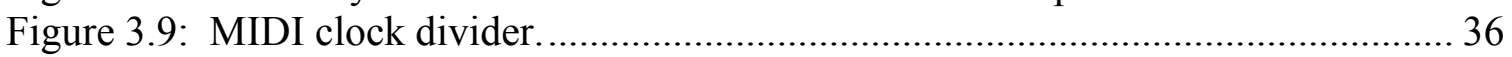

Figure 3.10: (a) Raspy vocal sample and (b) smooth vocal sample captured on a

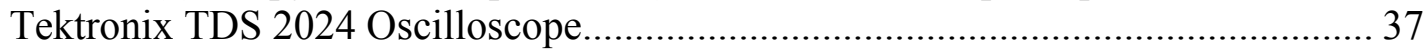

Figure 3.11: Amplification and filtering stages............................................................ 38

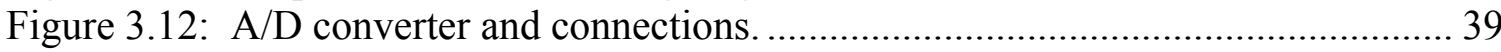

Figure 3.13: Divide-by 16 for sampling frequency generator. ....................................... 41

Figure 3.14: Adjustable sampling frequency generator............................................... 41

Figure 3.15: Interrupt acknowledge and MMU reset circuitry ...................................... 44

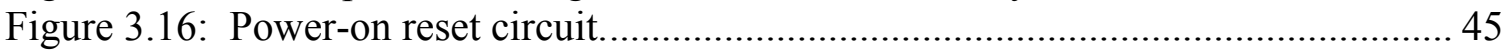

Figure 4.1: Topside view of the empty prototyping board. ........................................... 55

Figure 4.2: Topside view of loosely place parts on the prototyping board....................... 56

Figure 4.3: Flow diagram for interrupt service routine (ADCIRQ)............................... 58

Figure 4.4: Sample AMDF results (blue) and regions where the data fall below the

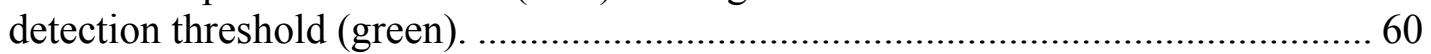

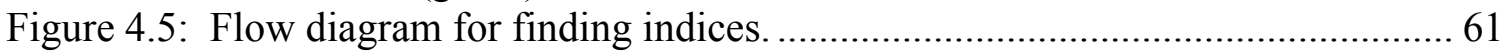

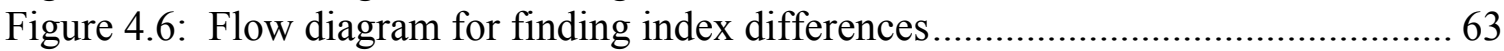

Figure 4.7: Flow diagram for sending MIDI commands............................................... 68

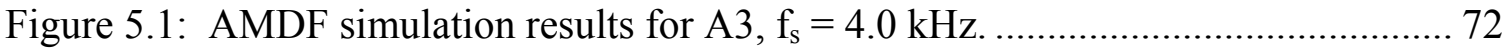

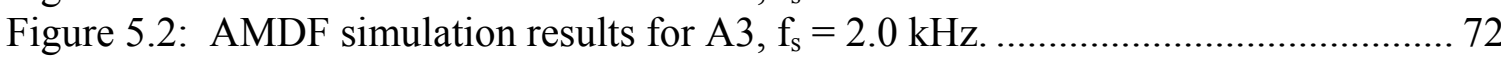

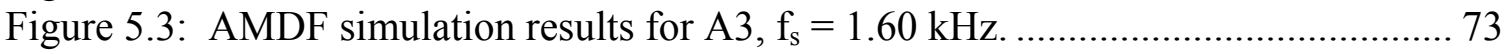

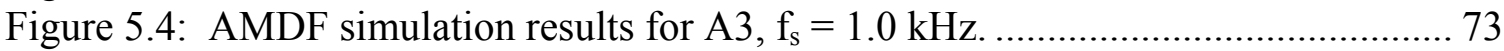

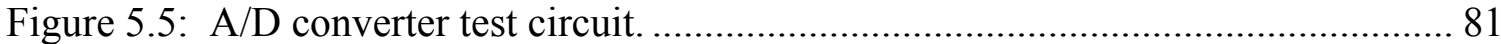

Figure 5.6: Fifth order Butterworth low pass filter design........................................... 84

Figure 5.7: Butterworth filter frequency responses for ideal (red, gently sloped) and actual (blue, steeply sloped) component values......................................................... 85 
Figure 5.8: Actual Butterworth filter frequency response with $100 \mathrm{~Hz} /$ division horizontally and $200 \mathrm{mV} /$ division vertically. The intersecting green and blue lines represent the $3.01 \mathrm{~dB}$ point.

Figure 5.9: Simulated Butterworth frequency response (blue) with updated L-values accounting for resistances. The (red) curve with the overall higher magnitude is the response with the ideal values

Figure 5.10: Simulated Butterworth frequency response with new L-values (blue). The (red) curve with the overall higher magnitude is the response with the ideal values.

Figure 5.11: Actual Butterworth frequency response using the new L-values with $100 \mathrm{~Hz} /$ division horizontally and $200 \mathrm{mV} /$ division vertically. The intersecting green and blue lines represent the $3.01 \mathrm{~dB}$ point 89 


\section{CHAPTER I - INTRODUCTION}

For anyone who has watched the first few episodes of each season of "American Idol" on the Fox television channel, it should be obvious that there are a number of Americans with singing difficulties. This results from the would-be singers lacking the ability or training to distinguish between small variations in pitch (frequency), and correct their vocal output accordingly. The vernacular calls this condition tone-deafness. However this term is actually incorrect, because tone-deafness by definition implies that one cannot hear tones, when in fact the difficulty lies in hearing the difference between tones. 'Research has shown that some people, termed 'amusic', can neither produce nor perceive music.” [Stewart, 2006] This condition called amusia is analogous to color blindness.

The original goal of this research was "to develop a device that will use the human voice to operate an electronic musical instrument" [Cleaver, 2000]. However, it was determined through calculation and experimentation that the original requirements of the design prototype would have to change due to hardware limitations (see Section 3.2.2). However, the original design will be presented in this section. Modifications will be presented and justified in subsequent sections where relevant.

The human voice will be sampled via a microphone and an analog to digital converter, which will be connected to a microprocessor that shall be programmed to operate as a digital signal processor. "The principal frequency ${ }^{1}$ of the input will be extracted, corrected for pitch and then used to operate the musical instrument. The implementation for the musical instrument will use the principal frequency data to

\footnotetext{
${ }^{1}$ The words "principal frequency" will herein be referred to as "fundamental frequency".
} 
provide keying commands to a commercial synthesizer keyboard" [Cleaver, 2000].

To use this product, the user must have access to a synthesizer capable of accepting and interpreting the MIDI protocol. The user must be able to make an audible tone from his/her vocal chords to activate the device. The device will be easy to operate in that there are only three setup requirements including: connecting the MIDI cable to the device and synthesizer, placing the microphone in an area close to the user's mouth, and supplying power to the synthesizer and device. Once these criteria are met, the user need only sing to operate the device.

The device must be able to identify vocal inputs in near real time so as not to be audibly noticeable. The device must be able to extract and output the fundamental frequency of the singing voice. The device shall be able to accommodate male and female singers. The device must be able to acquire the vocal signal without ambient noise, including the output of the synthesizer. The device shall be powered via an external power supply. The device must be able to communicate to a synthesizer via the MIDI protocol through a standard MIDI cable. The device must be able to communicate to a computer running a dummy terminal for development purposes. The device must be as small as reasonably possible. The device developed will be a functional prototype only and not a device suitable for manufacture, because the parts to be used to construct the prototype are no longer manufactured. However, the concepts that will be used to create the prototype can be expanded through further research to implement this device on modern technology.

The user interface for this device will consist of some knobs, switches, and indicators. The user shall use this interface to adjust the amplification settings of the 
microphone and operate the device. A possible user interface is show below in Figure 1.1, representing a possible view from the top of the device.

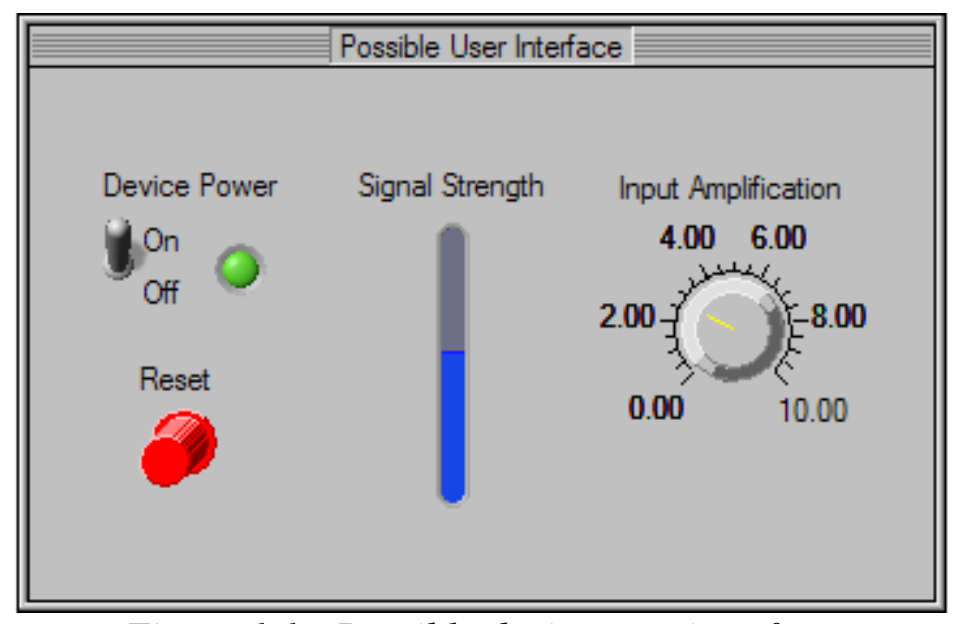

Figure 1.1: Possible device user interface.

The different items of interest are: the device power switch, the reset switch, the signal strength indicator, and the input amplification knob. The device power switch will be used to set the on or off state of the device. The (pushbutton) reset switch will primarily be used in development and emulation, but will be left for the user as alternate means to reinitialize the device. The signal strength indicator will indicate the amplitude of the amplified input signal from the microphone after the amplification and filtering stages. The input amplification control will consist of a knob that the user will adjust in conjunction with reading the signal strength indicator to optimize the input amplification of the microphone. 


\section{CHAPTER II - LITERATURE REVIEW}

The tasks involved in completing this project involve two main components: identifying the fundamental frequency and using the MIDI protocol to communicate appropriately to the synthesizer. In this chapter, the necessary background information is presented to complete these tasks. In Section 2.1, the elements of pitch and its perception are discussed. This is followed by a discussion of the impact of the human vocal range on this design in Section 2.2. Sections 2.3 and 2.4 discuss the techniques directly involved in identifying the pitch and communicating with the synthesizer, respectively.

\subsection{What is pitch?}

According to the ANSI standard for acoustical terminology, pitch, in a general sense can be defined as:

"...that attribute of auditory sensation in terms of which sounds may be ordered on a scale from low to high. Pitch depends mainly on the frequency content of the sound stimulus, but it also depends on the sound pressure and the waveform of the stimulus." [ANSI 1994]

The key words for this thesis are actually in the second sentence. Particularly, the dependence on the frequency content is of interest. However, the awareness that the stimulus and sound pressure can influence the pitch is also of interest, because this generally vague definition supports that the overall task of extracting the physical representation of the pitch (called the fundamental frequency) is subject to errors. The effects of some of these additional influences are discussed in Section 2.3.1.

In music, the dominant fields of study pertaining to this research are psychoacoustics and music psychology. According to Merriam-Webster's Medical Dictionary, the former is "a branch of science dealing with hearing, the sensations 
produced by sounds, and the problems of communication" while the latter is the study of how humans perceive musical elements and the feelings evoked by musical stimuli [Scheirer 2000].

"The fundamental frequency of a periodic signal is the inverse of its period, which may be defined as the smallest positive member of the infinite set of time shifts that leave the signal invariant. This definition applies strictly only to a perfectly periodic signal, an uninteresting object because it cannot be switched on or off or modulated in any way without losing its perfect periodicity." [Scheirer 2000]

Usually, the subjective recognition of a pitch associated with a sound depends on the fundamental frequency. However, there are exceptions to this generalization because some sounds may be periodic but have no pitch, while other sounds may not be periodic yet have a pitch [de Cheveigné 2003]. The classic example of the latter is that of a bell, which has a pitch, but no fundamental frequency [Gerhard 2003]. However, the relationship between the existence of pitch and a fundamental frequency is usually oneto-one insofar as the words "pitch" and "fundamental frequency" $\left(f_{0}\right)$ are used interchangeably in the field and in this document. A similar relationship exists between the terms " $f_{0}$ estimation methods" and "pitch detection algorithms" [de Cheveigné 2003].

\subsection{The human vocal range and the ideal choice of sampling rate}

The sampling rate is a crucial element in this design. According to the Shannon Sampling Theorem, the sampling rate must greater than or equal to twice the maximum frequency to be sampled. This minimum sampling rate is referred to as the Nyquist rate [Weeks 2007]. According the library of music at Yale University, the standard human vocal range is from E2 (bass singing at $82.407 \mathrm{~Hz}$ ) to A5 (soprano singing at $880.000 \mathrm{~Hz}$ ) 
[Yale 2005] and is illustrated in Figure 2.1. If this design is to accommodate the full spectrum of the human voice, the ideal sampling rate must then be $f_{s}=880 \cdot 2=1760 \mathrm{~Hz}$.

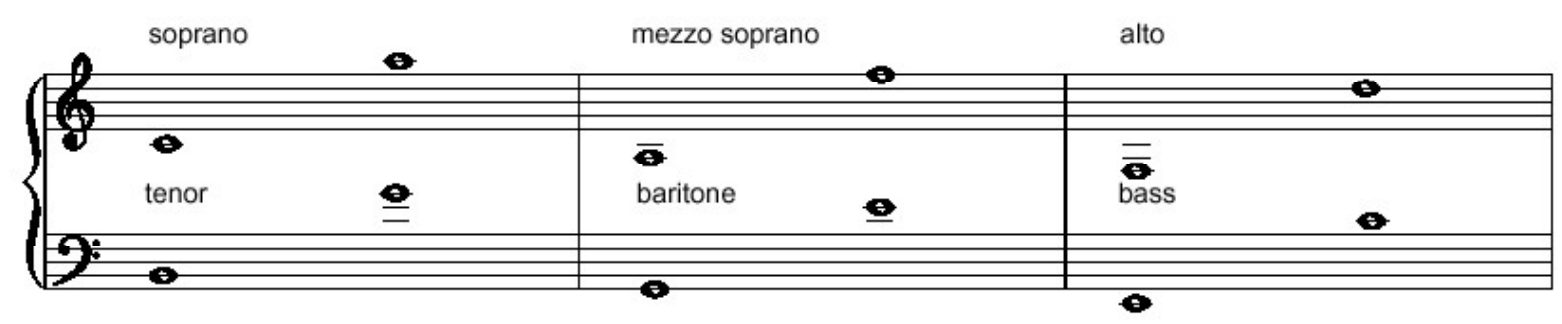

Figure 2.1: Vocal ranges for singers. Taken from [Yale 2005].

If the minimum sampling rate must be $1760 \mathrm{~Hz}$, a sampling rate of $2000 \mathrm{~Hz}$ would be chosen for this design for two reasons. First this is an easy number to implement in hardware via clock dividers, and this number is a convenient figure to use for multiplication. However, this sampling rate will be too high for this embedded software implementation to handle because of the system clock speed. These difficulties are discussed in greater detail in Section 3.1.

\subsection{Frequency Identification (Pitch-Tracking) Techniques}

There is a broad range of literature regarding extracting pitch information using a variety of different techniques as well as some research on real time vocal analysis and synthesis on a MIDI capable synthesizer (see [Ryynänen 2004, Saul 2002, and Shimamura 2001]). However, these algorithms and designs are designed to run on a personal computer (PC) and use sampling rates of $11.025 \mathrm{kHz}$ or higher. This device is unique in that it runs on an embedded system running at a much slower clock rate than a PC or its equivalent. The processor chosen for this project is the Motorola 68000 because 
of its availability in the lab. This processor can accept a clock in the neighborhood of 4$16 \mathrm{MHz}$; therefore, it is crucial that the algorithm be speed-efficient and fairly accurate.

There are a large variety of methods available to identify a frequency from a sample dataset. However, the hardware limitations govern the choice between the methods considered. This particular processor model does not support floating-point operations or complex mathematical functions such as: the exponential function, logarithms, sine, and cosine. Therefore, methods and algorithms requiring the use of such functions were dismissed. Many other methods other than those presented were reviewed but rejected for consideration because of these hardware limitations. Some of these methods are listed in Section 2.3.6, while other methods that warranted serious consideration are discussed in this section. The methods presented in this literature review are the primary methods investigated for this design following a brief discussion of some of the difficulties inherent to pitch tracking.

\subsubsection{Problems Associated with Pitch Detection of Vocal Signals}

In general, finding the frequency or period of a perfectly periodic waveform is relatively simple. However, measuring the pitch, or fundamental frequency, from a voiced signal is considered a difficult task mainly because when the glottis ${ }^{2}$ produces a waveform, the waveform is not a perfect composition of periodic pulses. It is also difficult to measure the interaction between the vocal tract and the glottal excitation

\footnotetext{
${ }^{2}$ The glottis is the space between one of the true vocal cords and the arytenoid cartilage on one side of the larynx and those of the other side [Merriam-Webster's Medical Dictionary].
} 
because the vocal tract formants ${ }^{3}$ can sometimes alter the glottal waveform structure. These interactions cause the most difficulty when the articulators change rapidly and also when the vocal formants themselves change rapidly [Rabiner 1976].

It is also difficult to determine the beginning and end of each pitch period in a voiced segment. This generally leads to the arbitrary choice of the pitch period beginning and ending times. An example of this arbitrary choice is shown in Figure 2.2. In this

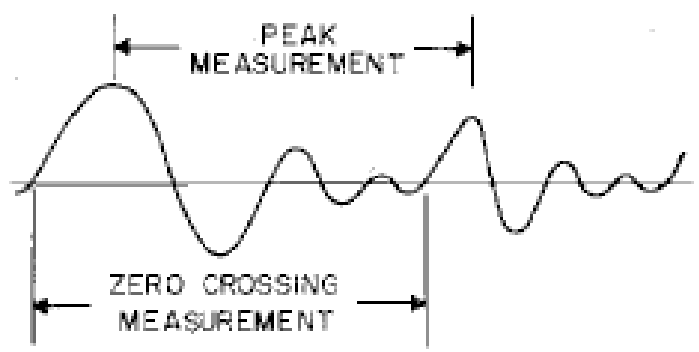

Figure 2.2: Two waveform measurements which can be used to define pitch markers. Taken from [Rabiner 1976].

figure, the two candidates for defining the period beginning/end are the maximum value and the zero-crossings prior to the maximum during each period. The only requirement with these measurements is that the locations be consistent from period-to-period, else spurious pitch estimates may result. In Figure 2.2, the period associated with the peak measurement will result in a higher frequency than the zero-crossing measurement. Discrepancies of this nature occur often because the speech waveform is quasi-periodic and because peak measurements are sensitive to formants, noise, and any DC level in the waveform. Another related difficulty arises from sorting between unvoiced speech and weakly voiced speech. This is problematic because the transitions between these two signal types are difficult to identify [Rabiner 1976].

${ }^{3}$ A formant is any of several frequency regions of relatively great intensity in a sound spectrum, which together determine the characteristic quality of a vowel sound [The American Heritage ${ }^{\circledR}$ Dictionary]. 


\subsubsection{Counting Zero-Crossings}

One of the simplest methods to measure frequency in the time domain is to measure the time between zero-crossings of the periodic signal. The reciprocal of the period corresponds to the frequency of interest. This is by far the easiest method to implement in hardware or software. However, this method is very susceptible to noise on the channel and any DC offset generated by the amplification or filtering stages in hardware, as well as the quantization error in the analog-to-digital (A/D) converter. This method is ideal for simple sinusoids, but a poor choice for complex waveforms with harmonics or distortions, such as vocal signals. To illustrate this point, look at Figure 2.3 below. On the left is a perfect sinusoid with frequency equal to $166.1 \mathrm{~Hz}$. On the right is a vocal sample at approximately the same frequency.

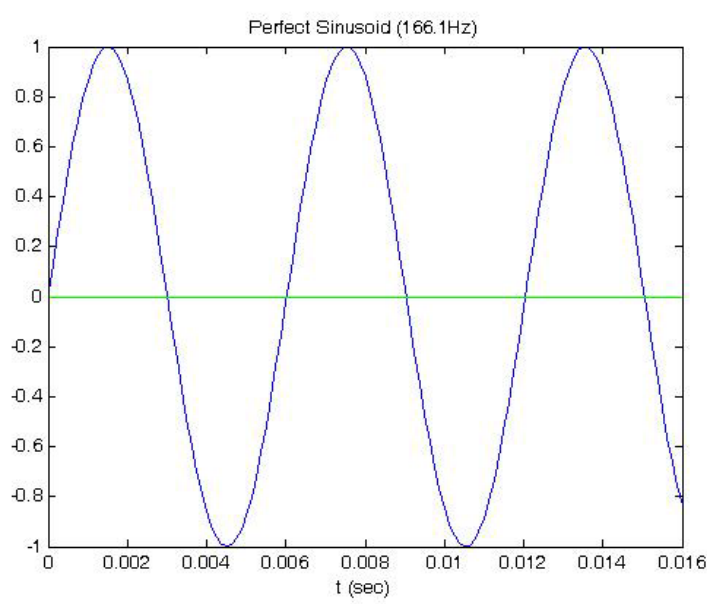

(a)

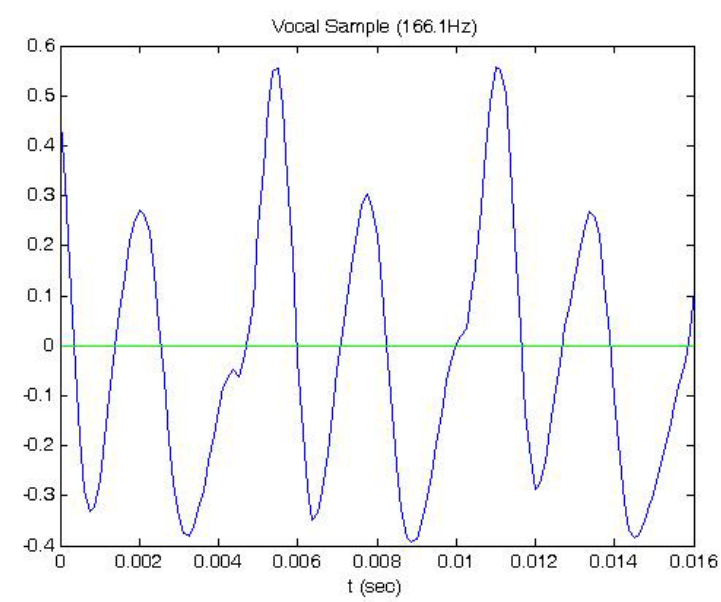

(b)

Figure 2.3: Perfect sinusoid (a) and vocal sample (b) at the same frequency.

If counting the number of zero crossings were used for the second figure, the frequency would be approximately twice the original frequency, because of the additional harmonics in the human voice. This method has been discarded from consideration; 
however, research is still performed today on finding better ways to use the zero-crossing method for pitch identification [Gerhard 2003].

\subsubsection{Frequency Domain Analysis using the Discrete Fourier Transform (DFT)}

Perhaps the more intuitive approach to identifying a fundamental frequency is to use the frequency domain for the analysis. The DFT "plays an important role in the analysis, design and implementation of discrete-time signal-processing algorithms and systems" [Oppenheim 1999]. The advantage of using the DFT is that the DFT spectrum is identical to samples of the continuous case of the Fourier transform with $\mathrm{N}$ spectra samples occurring at uniformly spaced frequencies, where the input signal is truly bandlimited. Many digital applications use the more efficient version of the DFT called the Fast Fourier Transform (FFT).

Frequency domain analysis is used frequently in conjunction with tools such as a spectrum analyzer or an oscilloscope with an FFT implementation, which typically have some form of a fast digital signal processing IC to perform the calculations. Other systems similar to this research may use variations of the FFT, such at decimation in time, decimation in frequency, or other special implementations such as Cooley-Tukey's, the Prime-factor, Bruun's, Rader's, or Bluestein's FFT algorithms to find spectral results [FFT, 2007].

In theory, the implementation of this algorithm would find the maxima of the power spectra to identify the frequency once the data are transformed into the frequency domain. Figure 2.4 represents the vocal data from Figure $2.3 \mathrm{~b}$ in the frequency domain. In this figure, it is clearly obvious that the three harmonics have sufficient power to be 
identified as the fundamental frequency. In fact, the middle spike has the highest power, but it does not correlate with the pitch produced by the test subject.

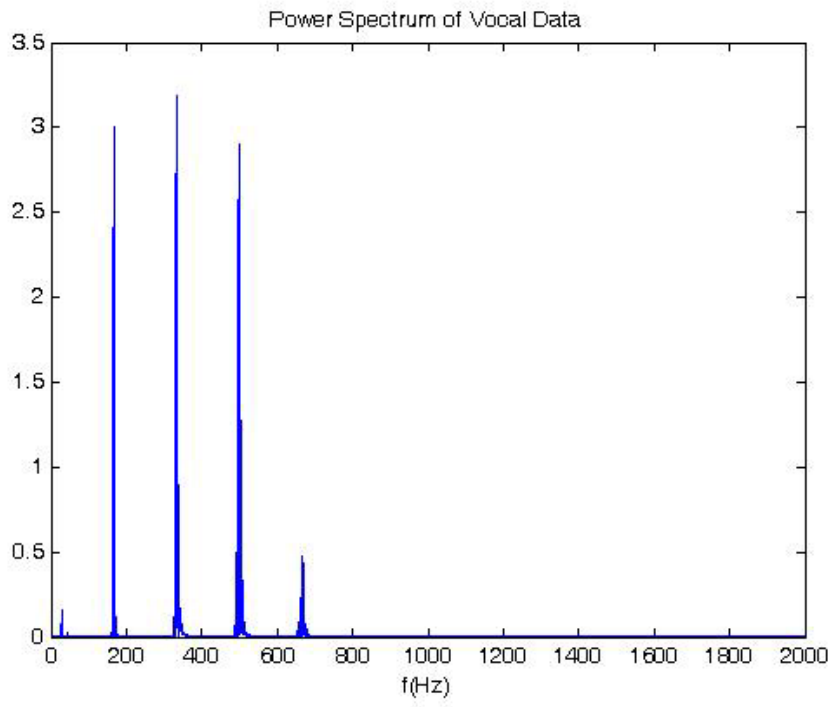

Figure 2.4: Power spectrum of the vocal sample from Figure 2.3b.

The problem with this method is that the number of sample points, N, would have to be a 512 or 1024 point DFT/FFT to get a $1-2 \mathrm{~Hz}$ resolution on the frequency axis, which would take too long to compute in real time. The Motorola website had an FFT code example that was written by Ron Williams from Ohio University, but based on code appearing in Byte Magazine in 1979. As of November 27, 2007, the code is currently

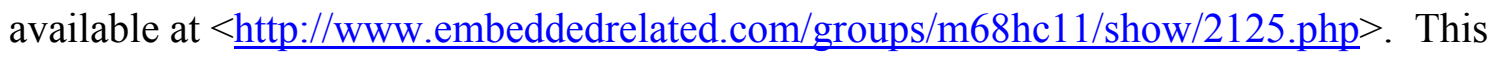
code is designed to run on the Motorola $68 \mathrm{HC} 11$ (2MHz system clock) and computes a 256 point 8-bit integer FFT. The reported execution time of this code is $350 \mathrm{~ms}$. Performance estimation for the 68000 based on this code is possible with the proper considerations. First, the execution time can be divided by four since the 68000 's system clock is $8 \mathrm{MHz}$. However, the time must be rescaled to accommodate for the larger FFT. The computational complexity of the FFT is $\mathrm{O}(N \log N)$. So if $87.5=N \log N$, then $N=$ 
26.653 and $2 N=53.306$. Thus $53.306 \cdot \log (53.306)=211.949 \mathrm{~ms}$, which is too long to execute a 512 point FFT for real time applications.

\subsubsection{Auto-Correlation Function}

Shimamura and Kobayashi have done research in the area of extracting pitches from noisy speech signals by using the auto-correlation function (ACF) and the average magnitude-difference function (AMDF) [Shimamura 2001]. The ACF is based in the time domain and is defined by Equation 2.1:

$$
\phi(\tau)=\frac{1}{N} \sum_{n=0}^{N-1} x(n) x(n+\tau)
$$

where:

$$
\begin{aligned}
& x(n)=\text { vocal signal sample; } \\
& \tau=\text { the lag number, or time shift; } \\
& n=\text { the time for a discrete signal }
\end{aligned}
$$

Sliding a small window of the sampled signal with the whole of the sampled data, in essence, forms the ACF results. As periodic segments overlap the similar segments of the sampled data, $\phi(\tau)$ assumes a large value at integer multiples of the signal's fundamental period $\left(\mathrm{T}_{0}\right)$. The fundamental period is calculated from the differences in $\tau$ corresponding to the peaks of $\phi(\tau)$; dividing the sampling rate by the period yields the fundamental frequency $\left(f_{0}\right)$.

The advantages of the ACF are obvious in that the ACF does not require the use of exponentials, logarithms or sinusoidal functions to perform the calculation. The math is straightforward and simple. Shimamura and Kobayashi prove for a large N, that if a noisy speech signal $\mathrm{x}(\mathrm{n})$ is composed of a clean speech signal, $\mathrm{s}(\mathrm{n})$ and additive white 
Gaussian noise, w(n), s(n) will not correlate with $w(n)$. Therefore, an added advantage of the $\mathrm{ACF}$ is that it performs well in noisy environments.

The problem with the ACF is that sometimes the peak located at the second multiple of $\mathrm{T}_{0}$ is much larger than the first peak. This can lead to a half-pitch error in frequency identification. In instances where the first peak occurs at a time of $\tau<\mathrm{T}_{0}$, a double-pitch error in frequency identification may occur [Shimamura 2001].

\subsubsection{Average Magnitude-Difference Function (AMDF)}

The AMDF is similar to the ACF in that the results are obtained by sliding a small window of the sampled signal with the whole of the sampled data. Equation 2.2 represents the AMDF:

$$
\psi(\tau)=\frac{1}{N} \sum_{n=0}^{N-1}|x(n)-x(n+\tau)|
$$

where $\mathrm{x}(\mathrm{n}), \tau$ and $\mathrm{n}$ correspond to their counterparts in the definition of the ACF in Equation 2.1.

The primary difference between the two formulas is that as periodic segments overlap the similar segments of the sampled data, $\psi(\tau)$ assumes small values at integer multiples of the signal's fundamental period $\left(\mathrm{T}_{0}\right)$. The fundamental period is calculated from the differences in $\tau$ corresponding to the notches of $\psi(\tau)$; dividing the sampling rate by the period once again yields the fundamental frequency $\left(f_{0}\right)$.

Shimamura and Kobayashi also prove for a large N, that if a noisy speech signal $\mathrm{x}(\mathrm{n})$ is composed of a clean speech signal and additive white Gaussian, the signal and noise are independent. Thus, the AMDF also performs well in noisy environments. The AMDF shares the advantages of the ACF as described above [Shimamura 2001]. 
However, the AMDF is advantageous over the ACF for this design because the main operation is subtraction and the absolute value function, whereas the main operation in the ACF is multiplication. On the 68000 , and most processors, multiplication requires more clock cycles to execute than subtraction. This processor's execution time varies for mathematical functions depending on values of the input arguments. The worst-case scenario for implementing the subtraction and absolute value functions ( 20 clock cycles) is less than the best-case scenario (multiplying zero by zero) for unsigned multiplication (38 clock cycles) [Motorola 1993]. This means that the ACF, at its best will require 90\% more time to execute. Additionally, the AMDF has a sharper pitch resolution when compared to the results of the ACF [Kim 1998].

$$
\operatorname{AMDF}(n)=\sum_{k=0}^{N-1}|s(k)-s(k+n)|
$$

Therefore, the ADMF will be implemented for pitch detection in this design, with the exception that it will not be normalized with respect to the total number of samples $(\mathrm{N})$ and is defined in Equation 2.3. The reason the normalization step is excluded is that dividing by $\mathrm{N}$ wastes clock cycles, but more importantly, the 68000 does not handle floating-point numbers as easily as 16-bit integers. Since scaling the data essentially has no added effect for this application, its removal is justified. Although this change would more effectively lend the algorithm to be called the Sum of Magnitudes Difference Function, it will still be referred to as the Average Magnitude Difference Function to be consistent with the literature available. 


\subsubsection{Some Other Techniques Worth Mentioning}

In 1976, Lawrence Rabiner et al. authored a paper for the IEEE that comparatively studied several pitch detection algorithms. Their studies included both performance in terms of accuracy of the detection as well as computation time. Each algorithm was implemented on the same data sets consisting of a low-pitched male (LM), two male speakers (M1 and M2), two female speakers (F1 and F2), a child (C1) and a diplophonic $^{4}$ speaker (D1). All filtering, signal conditioning and signal processing was handled digitally on a Nova 800 minicomputer. The algorithms they studied were the ACF using clipping (AUTOC), the cepstrom method (CEP), the simplified inverse filtering technique (SIFT), the data reduction method (DARD), the parallel processing method (PPROC), the spectral equalization LPC method using Newton's transformation (LPC) and the previously discussed AMDF.

Table 2.1 shows the computational performance results for the different algorithms studied by Rabiner et al. For each algorithm, the speed was computed from processing a one second sample set. In these results, the AMDF had the third best performance. However, Tables 2.2 and 2.3 show that the AMDF significantly outperforms the faster two algorithms in terms of accuracy. The remaining algorithms, although more accurate, require significantly more time to compute the results, these algorithms were not considered for implementation.

\footnotetext{
${ }^{4}$ diplophonia is a condition in which the voice simultaneously produces two sounds of a different pitch [Dictionary.com]
} 


\begin{tabular}{|c|c|c|c|c|}
\hline $\begin{array}{c}\text { Pitch } \\
\text { Detector }\end{array}$ & $\begin{array}{l}\text { Speed/s of } \\
\text { Speech }\end{array}$ & $\begin{array}{l}\text { Arithmetic } \\
\text { Type }\end{array}$ & $\begin{array}{l}\text { Down- } \\
\text { sampling } \\
\text { Used }\end{array}$ & $\begin{array}{l}\text { Dependence on } \\
\text { Sampling Rate }\end{array}$ \\
\hline DARD & $5 \mathrm{~s}$ & Integer & No & Linear \\
\hline PPROC & $7.5 \mathrm{~s}$ & Integer. & No & Linear \\
\hline AMDF & $50 \mathrm{~s}$ & Floating point & $\mathrm{No}^{\mathrm{a}}$ & Quadratic \\
\hline AUTOC & $120 \mathrm{~s}$ & Integer & $\mathrm{No}^{\mathrm{a}}$ & Quadratic \\
\hline SIFT & $250 \mathrm{~s}$ & Floating point & Yes & Quadratic \\
\hline LPC & $300 \mathrm{~s}$ & Floating point & Yes & Quadratic \\
\hline CEP & $400 \mathrm{~s}$ & Mixed & No & Linear \\
\hline
\end{tabular}

Table 2.1: Computational considerations for the seven pitch detectors on the Nova 800 minicomputer. Taken from [Rabiner 1976]. 


\begin{tabular}{|c|c|c|c|c|c|c|c|c|}
\hline \multicolumn{9}{|c|}{ Pitch Detector } \\
\hline \multicolumn{2}{|c|}{ Speaker } & \multirow{2}{*}{$\begin{array}{c}\text { AUTOC } \\
15.3\end{array}$} & \multirow{2}{*}{$\begin{array}{r}\text { CEP } \\
0.5\end{array}$} & \multirow{2}{*}{$\begin{array}{r}\text { SIFT } \\
0.6\end{array}$} & \multirow{2}{*}{$\begin{array}{c}\text { DARD } \\
5.8\end{array}$} & \multirow{2}{*}{$\begin{array}{c}\text { PPROC } \\
10.0\end{array}$} & \multirow{2}{*}{$\begin{array}{r}\text { LPC } \\
4.4\end{array}$} & \multirow{2}{*}{$\begin{array}{r}\text { AMDF } \\
12.8\end{array}$} \\
\hline LM & $\mathbf{M}$ & & & & & & & \\
\hline & $T$ & 26.1 & 1.1 & 4.5 & 5.8 & 11.0 & 5.6 & 15.6 \\
\hline & W & 19.5 & 1.3 & 4.5 & 13.8 & 23.8 & 13.0 & 23.8 \\
\hline & Sum & 60.9 & 2.9 & 9.6 & 25.4 & 44.8 & 23.0 & 52.2 \\
\hline \multirow[t]{4}{*}{ M1 } & M & 0.6 & 0.1 & 0.0 & 5.9 & 2.0 & 0.1 & 0.3 \\
\hline & $\mathrm{T}$ & 3.4 & 0.1 & 0.8 & 6.3 & 3.0 & 0.8 & 0.8 \\
\hline & W & 2.8 & 0.5 & 3.0 & 23.5 & 6.0 & 0.8 & 2.8 \\
\hline & Sum & 6.8 & 0.7 & 3.8 & 35.7 & 11.0 & 1.7 & 3.9 \\
\hline \multirow[t]{4}{*}{ M2 } & M & 6.1 & 0.4 & 1.3 & 15.9 & 4.9 & 2.9 & 7.3 \\
\hline & $T$ & 9.9 & 0.6 & 3.4 & 4.0 & 5.8 & 4.0 & 9.8 \\
\hline & W & 7.3 & 1.3 & 5.3 & 26.8 & 12.3 & 5.5 & 8.5 \\
\hline & Sum & 23.3 & 2.3 & 10.0 & 46.7 & 23.0 & 12.4 & 25.6 \\
\hline \multirow[t]{4}{*}{ F1 } & M & 1.9 & 9.1 & 4.4 & 7.3 & 4.0 & 2.4 & 0.5 \\
\hline & $\mathbf{T}$ & 1.6 & 8.5 & 1.8 & 6.3 & 2.8 & 1.4 & 0.0 \\
\hline & W & 0.0 & 29.0 & 8.0 & 0.8 & 4.0 & 2.0 & 0.0 \\
\hline & Sum & 3.5 & 46.0 & 14.2 & 14.4 & 10.8 & 5.8 & 0.5 \\
\hline \multirow[t]{4}{*}{ F2 } & $\mathbf{M}$ & 0.4 & 1.4 & 2.1 & 7.1 & 2.4 & 1.6 & 0.6 \\
\hline & $T$ & 0.6 & 2.0 & 1.5 & 5.6 & 1.5 & 1.5 & 1.0 \\
\hline & W & 2.0 & 2.5 & 3.8 & 8.5 & 5.0 & 2.0 & 1.8 \\
\hline & Sum & 3.0 & 5.9 & 7.4 & 21.2 & 8.9 & 5.1 & 3.4 \\
\hline \multirow[t]{4}{*}{$\mathrm{C} 1$} & $\mathbf{M}$ & 1.0 & 13.6 & 65.3 & 6.1 & 7.8 & 8.3 & 10.6 \\
\hline & $T$ & 1.9 & 14.8 & 62.6 & 12.9 & 9.0 & 12.3 & 9.1 \\
\hline & W & 0.0 & 12.5 & 40.8 & 3.0 & 7.3 & 5.5 & 6.5 \\
\hline & Sum & 2.9 & 40.9 & 168.7 & 22.0 & 24.1 & 26.1 & 26.2 \\
\hline
\end{tabular}

Table 2.2: Number of gross pitch errors - unsmoothed. Taken from [Rabiner 1976].

\begin{tabular}{|c|c|c|c|c|c|c|c|c|}
\hline \multicolumn{9}{|c|}{ Pitch Detector } \\
\hline Speaker & AUTOC & CEP & SIFT & DARD & PPROC & LPC & AMDF & Sum \\
\hline LM & 4 & 1 & 2 & 3 & 4 & 3 & 4 & 21 \\
\hline M1 & 2 & 1 & 1 & 3 & 2 & 1 & 1 & 11 \\
\hline M2 & 3 & 1 & 2 & 4 & 3 & 2 & 3 & 18 \\
\hline F1 & 1 & 4 & 2 & 2 & 2 & 1 & 1 & 13 \\
\hline F2 & 1 & 1 & 2 & 3 & 2 & 1 & 1 & 11 \\
\hline $\mathrm{C} 1$ & 1 & 3 & 5 & 3 & 3 & 3 & 3 & 21 \\
\hline Sum & 12 & 11 & 14 & 18 & 16 & 11 & 13 & \\
\hline
\end{tabular}

Table 2.3: Performance scores based on sum of gross pitch errors - unsmoothed. Taken from [Rabiner 1976].

\subsubsection{Anticipated Problems with the AMDF}

Based on the literature review, the AMDF is susceptible to three main problems with this application. The first is the sampling rate. Although the results by Rabiner et al. show the AMDF is fairly accurate, they also used pre-recorded data and a sampling 
frequency of $11.025 \mathrm{kHz}$ to perform their study. Even though the AMDF can be downsampled successfully [Rabiner 1976], the sampling frequency implemented in this design is extremely small compared to that used in other research in the field. Thus it is anticipated that errors will occur as a result of this reduced sampling rate.

The second concern is that the AMDF is known to produce octave, or pitchdoubling errors when a fixed threshold for period detection is used. This is also a problem with other algorithms and can easily been seen again by recalling Figure 2.4. In [Kim 1998], they demonstrate that an adaptive threshold can be used to determine when periods occur. By using a weighting factor and comparing other suitable pitches by doubling, tripling, quadrupling, etc... the proposed fundamental frequency, they see if each multiple of the original pitch falls under the proposed threshold. If so, a new pitch is identified [Kim 1998]. Although ideal, implementing an adaptive threshold will not be possible in this design because it requires too much time to execute.

Regarding octave errors, the real time processing can be advantageous because this concern can be mitigated by the fact that this design is to be used by amateur singers who are less likely to jump more than an octave unintentionally. Tracking the numerical difference between MIDI note numbers will easily reveal if a singer tries to jump more than an octave at a time, which will help in preventing octave errors. However, if the initial pitch detection is too high, the user will not be able to jump to a lower octave.

The last known problem is identifying a pitch when the user changes between notes. It is difficult to know when one note ends, and if the sampling routines acquire a sample set during a transition, the reported pitch will be inaccurate. 


\subsection{The MIDI Protocol}

Composers and musicians have used the Musical Instrument Digital Interface (MIDI) protocol since its development in 1983 . The protocol was originally designed so musicians could connect synthesizers together. Today, the protocol is used to supplement audio in gaming and multimedia applications due to the extremely small file size required to create a MIDI file versus a sampled audio file [MMA 2001]. The size of the files is roughly analogous to comparing a vector drawn image versus a bitmap image of the same object.

The MIDI protocol makes it available not only for communication between synthesizers, but also between other sound modules, wind controllers, guitars and the modern personal computer [MMA 2007]. This design will make use of the small packet size to control a synthesizer, and will use unidirectional communication between the prototype and the synthesizer.

\subsubsection{Message Format}

The MIDI message format is quite simple. The beginning of a new message contains a status nibble, followed by a channel number nibble (where $0-15_{\text {dec }}$ corresponds to channels $\left.1-16_{\mathrm{dec}}\right)$. The remaining size of the transmitted data depends on the command used. The MIDI protocol specifies that any other transmitted data must only utilize the lower seven bits of a byte. Placing this restriction ensures that the MIDI device can always detect a new command, ' $\mathrm{s}$ ' (see below), which always utilizes the most significant bit of the upper nibble. Only three of the commands are used in this design. They are Note On, Note Off, and Program Change. The packet format for turning a note on or off is as follows: 


$$
\begin{aligned}
s= & \text { Status Nibble (Command) } \\
& 8=\text { Note Off } \\
& 9=\text { Note On } \\
c= & \text { Channel Number }\left(0-15_{\mathrm{dec}}\right) \\
\mathrm{nn}= & \text { MIDI Note Number }\left(0-127_{\mathrm{dec}}\right) \\
\mathrm{vV}= & \text { Note Velocity }
\end{aligned}
$$

Example MIDI Packet (hex numbers):

$90457 \mathrm{~F}$ - Play pitch A4 $(440 \mathrm{~Hz})$ on channel 1 with velocity 127

$803 \mathrm{C} \odot \odot$ - Stop pitch $\mathrm{C} 4$ on channel 1, velocity number irrelevant

The packet format for changing the MIDI instrument is as follows:

$$
\text { sc pp }
$$

$$
\begin{aligned}
s= & \text { Status Nibble (Command) } \\
& 12_{\mathrm{dec}}=\text { program change } \\
c= & \text { Channel Number }\left(\odot-15_{\mathrm{dec}}\right) \\
\mathrm{p}= & \text { New instrument number }\left(\odot-127_{\mathrm{dec}}\right)
\end{aligned}
$$

Example MIDI Packet (hex numbers):

C๑ $\odot \odot$ - Change current instrument on channel 1 to Acoustic Grand Piano

There are seven total MIDI commands that are represented in hexadecimal per the table below. The last hexadecimal value, F, is reserved for future development and will be ignored by the MIDI device if transmitted [MMA 1995],[MMA 1995]. Table 2.4 contains the relevant commands for this design.

\begin{tabular}{|c|l|}
\hline Hex & \multicolumn{1}{|c|}{ Command } \\
\hline 8 & Note off \\
\hline 9 & Note on \\
\hline A & After touch (key pressure) \\
\hline B & Control change \\
\hline C & Program (instrument) change \\
\hline D & Channel pressure \\
\hline E & Pitch wheel \\
\hline \multicolumn{2}{|c|}{ Table 2.4: MIDI commands }
\end{tabular}

\subsubsection{Electrical Specifications}


The MIDI data transmission protocol uses the RS-232 standard. The bit-rate is $31250 \mathrm{bps}$, and the voltages range from $0-5 \mathrm{~V}$ representing a logic $0-1$, respectively [MMA 2001]. The MIDI cable itself requires a shielded cable and cannot exceed a length of 50ft (15m) [MMA 1985] and consists of two different connectors (MIDI In and MIDI Out), and sometimes a third (MIDI Thru) [MMA 2001]. Cables designed to interface with a PC sound card using a DB15 connection, and contain an adapter with components similar to the Figure 2.5.

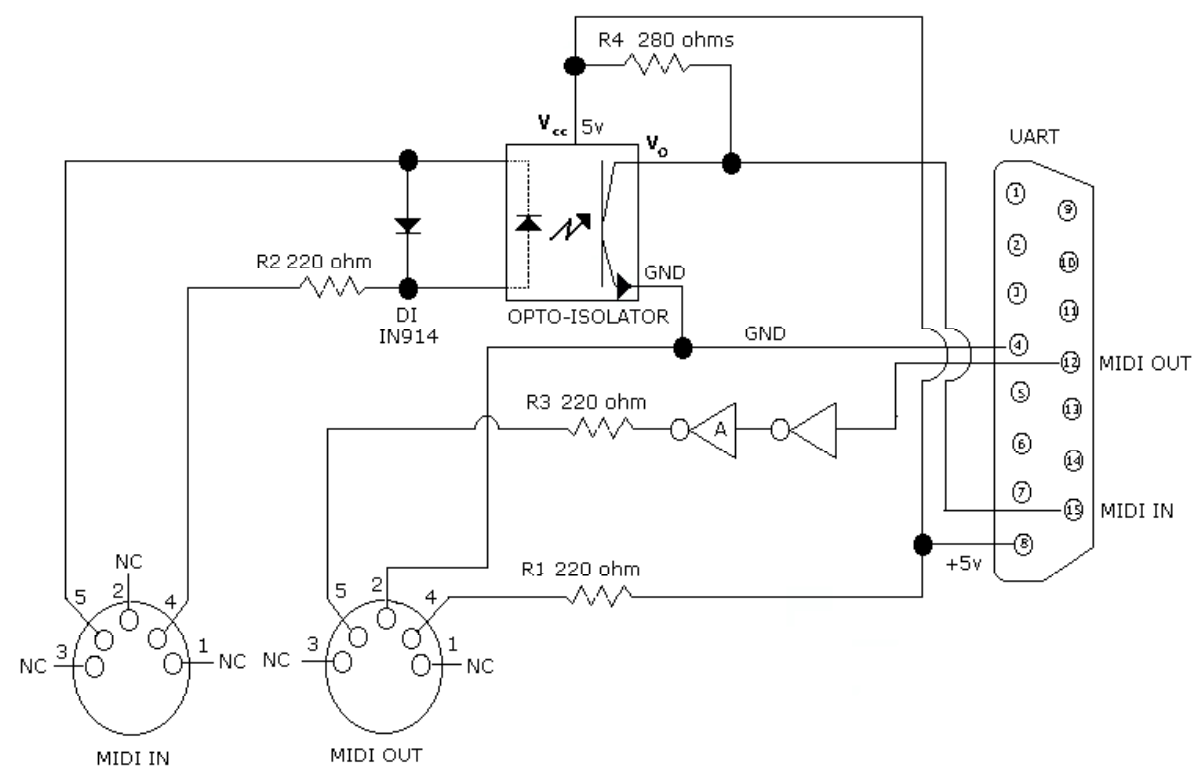

Figure 2.5: Computer Sound Card Game Port to Standard MIDI Connector [MMA 1985].

This design makes use of the DB15 connector cable above, as it requires the least amount of external components to implement. 


\section{$\underline{\text { 2.4.3 Timing Considerations }}$}

MIDI was designed to convey musical performance data and therefore preserves rhythmic integrity of the music by using accurate timing. A standard RS232 packet consists of ten bits to transmit one byte of data (consisting of one start bit, eight data bits, one stop bit, and no parity) for this application. Sending a simple three-byte message for a Note On or Note Off command will only take $0.96 \mathrm{~ms}$ to transmit. Even with larger packets, the delay between sending the packet and hearing the sound (latency) is usually $3 \mathrm{~ms}$ or less, depending on the size of a packet. Research has shown that 20-30 ms latency is usually imperceptible, so long as the variation in latency (the jitter) is small [Lago 2004]. Usually, the jitter associated with the MIDI protocol is less than $1 \mathrm{~ms}$.

However, it is important in this design to consider the time it takes to transmit the MIDI data. In this application, only one musical part is produced at once with small data packets, therefore the MIDI protocol timing should be quick enough and should be rhythmically accurate [MMA 2001]. 


\section{CHAPTER III - SYSTEM DESIGN}

\subsection{Description of the Hardware}

The hardware for this design can be broken down into four basic modules consisting of the hardware responsible for sampling, serial communications, MIDI communications, and memory management. Each of these large modules consists of smaller, supporting modules, and each of the larger modules is connected with the 68000 . Each of the major modules is briefly described here and the detailed description will follow.

The first module is the sampling module. It consists of the microphone, amplification stage, filtering stage, the A/D converter, and the clock providing the conversion rate for the A/D converter. Another clocking mechanism is directly interfaced with the 68000 to trigger the sampling routine.

The second module is the serial communications interface (SCI) module. This module was originally designed for a developer. As implementation problems developed, the concept of adding a developer software interface was abandoned, but the fully functional hardware was left on the final prototype; this is the only unused interface in this design. This module consists of an asynchronous communications interface adapter (ACIA), a bit-rate generator, and a voltage level shifter.

The third module is the MIDI communications module, which is used to interface to the synthesizer directly. This module also consists of an ACIA and uses a simple clock divider (counter) connected to the system clock to act as a bit-rate generator. The serial output of the MIDI ACIA is connected to the MIDI OUT input to the synthesizer. 
The final module is the memory management unit (MMU). It is responsible for enabling the various devices for read and/or write access as well as indicating to the 68000 when a data transmission is acknowledged (DTACK') and when valid peripherals request use of the address bus (VPA').

\subsubsection{M68000 Connections}

The Motorola M68000 is the microprocessor used in this design. There are two practical choices for a processor for this design based on hardware and test equipment that are readily available at the engineering school. These two choices are the Motorola M68HC11 microcontroller and the Motorola M68000. The latter was chosen over the Motorola M68HC11 because of speed, memory capacity, and that the M68000 easily facilitates connecting a large number of external devices.

The address and data line connections are described in detail in the memory management unit and memory sections in sections 3.1 .2 and 3.1.3, respectively, as well as other sections where appropriate. However, address lines A17-A23 are left disconnected, as they are not used.

The address strobe (AS)' is an output that indicates the address bus is ready for use. It is connected to the memory management unit (MMU) and interrupt acknowledge (IACK') circuitry to enable communications with memory and to identify interrupt requests, respectively. The data transmission acknowledge (DTACK') input is connected to DTACK' from the MMU (Section 3.1.4), and the valid peripheral address (VPA') input is connected to the VPA' circuitry (Section 3.1.5) to indicate when memory and peripherals are ready to transmit/receive data. The upper and lower data strobes (UDS'/LDS') and the read/write (R/W') line are connected to the MMU to indicate 
whether an even or odd address is being accessed and whether a read or write cycle is engaged. The EN clock, which divides the system clock by ten, is connected as ECLK to the two M6800 series peripheral devices (M6850s) and the A/D converter clock. Finally, the valid memory address (VMA') output is negated and is used to signal to the two peripheral addresses that the 68000 has recognized a valid peripheral address (VPA') created by selecting one of those M6800 series peripherals (Sections 3.1.7 and 3.1.8).

The system clock (CLK) input comes directly from an $8 \mathrm{MHz}$ TTL oscillator. The function code pins (FC0-FC2) are connected to the interrupt acknowledge (IACK') circuitry to indicate an interrupt cycle on the 68000 (Section 3.1.11). These three pins are all asserted when an interrupt request is acknowledged. IPL0' is connected to the sampling frequency (FS') clock to trigger a level one interrupt request (Section 3.1.10). The HALT' and RST' lines are connected to the main reset signal (RESET') generated by the power-on reset circuitry (Section 1.1.12). The IPL1', IPL2', BERR', BGACK' and BR' lines are unused and are tied high to $\mathrm{V}_{\mathrm{CC}}$ via a $4.7 \mathrm{k} \Omega$ resistor. The bus grant (BG') output is left disconnected.

\subsubsection{The Memory Management Unit (MMU)}

The MMU, shown in Figure 3.1, is responsible for selecting between two NVRAMs, one latch, one A/D converter, and two ACIAs for read and/or write operations. The MMU consists of two 74LS138 decoders and a Schmitt triggered inverter (74LS14) to perform device selection and some AND gates to create the DTACK' signal (Section 3.1.4). 


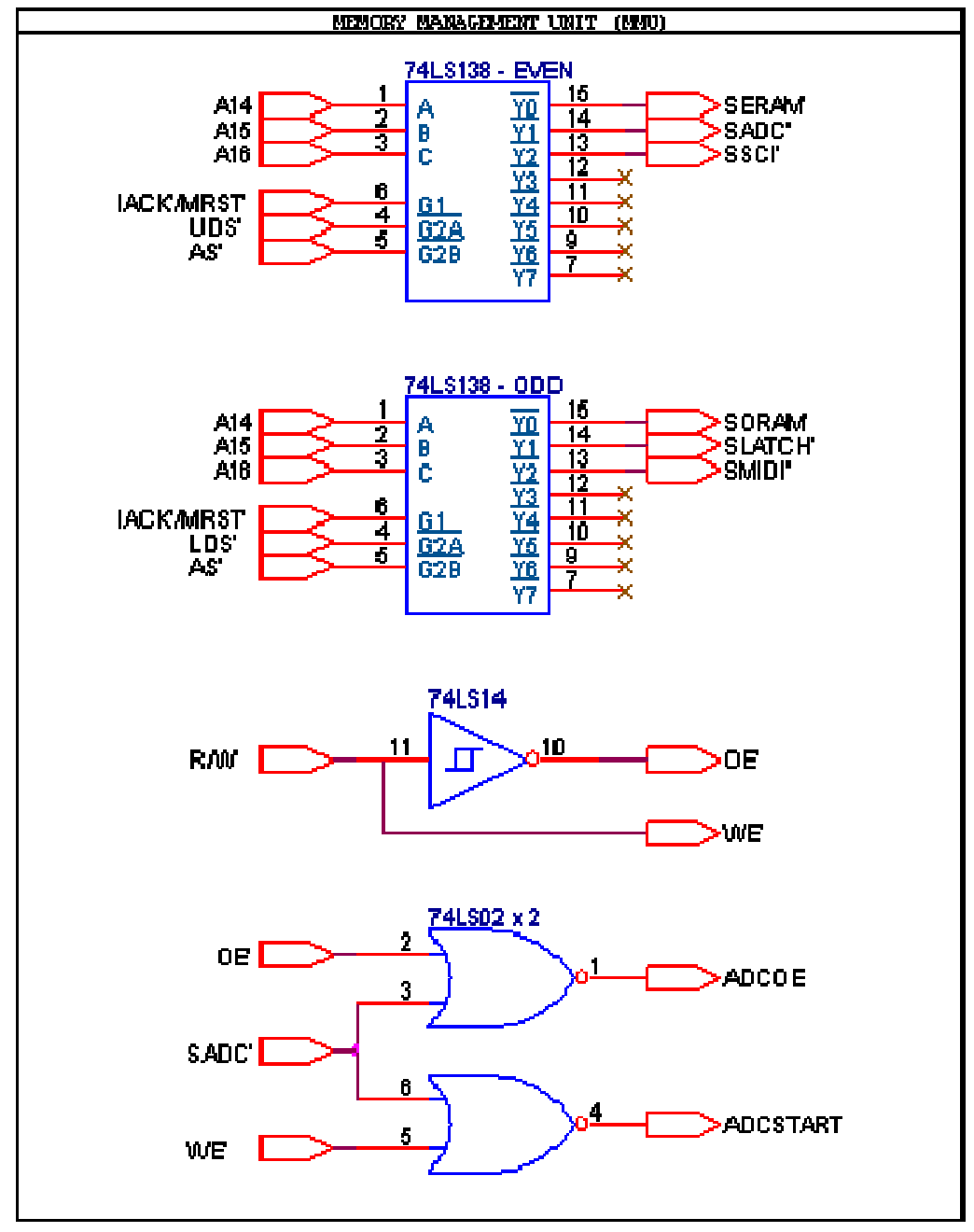

Figure 3.1: Memory management unit.

Address lines A14-A16 are connected from the 68000 to the select inputs A-C, respectively, on each decoder. When the decoders are enabled, these lines are responsible for selecting between the six devices previously mentioned. The active-high enable (G1), of each decoder is connected to the active-low IACK'/MRST' signal. This signal serves to disable the MMU during reset and to prevent erroneous data from being written to the NVRAMs during power-on. This signal also disables the MMU during the 68000's interrupt acknowledge sequence and is discussed in Section 3.1.11. Address strobe (AS') from the 68000 is connected to G2B' of each decoder. Finally, the upper 
data strobe (UDS') and lower data strobe (LDS') signals are connected to G2A' of the even and odd decoders, respectively. Thus, when AS' and UDS'/LDS' are asserted and IACK'/MRST' is negated, the MMU selects between devices for I/O operations.

On the even decoder (top of Figure 3.1), the active-low outputs Y0', Y1' and Y2' become the control signals SERAM', SLATCH', and SSCI', respectively. These control signals respectively select between the even RAMs, the latch used for operating the LEDs and the serial communications interface. On the odd decoder (below the even decoder in Figure 3.1), the active-low outputs Y0', Y1' and Y2' become the control signals SORAM', SADC', and SMIDI', respectively. These control signals respectively select between the odd RAMs, the A/D converter, and the MIDI ACIA. All other outputs (Y3'Y7') on both decoders are left disconnected.

The $\mathrm{R} / \mathrm{W}$ ' signal from the 68000 is inverted to create the active-low output enable signal (OE') for use on the NVRAMs. The Schmitt inverter package is used for the power-on reset, and is used here to save board space since the functionality is essentially the same for standard logic levels and operations. The active-low write enable signal (WE') is directly connected to the $\mathrm{R} / \mathrm{W}^{\prime}$ signal on the 68000 .

All the signals output from the decoders are active-low. However, the A/D converter requires active-high control signals and some special handling since there is no chip enable input on the A/D converter. Therefore, OE' and SADC' from the MMU are NORed and form the A/D output enable (ADCOE) signal. Similarly, WE' and SADC' from the MMU are NORed and for the A/D start conversion (ADCSTART) signal. With this configuration, the $\mathrm{A} / \mathrm{D}$ converter will begin a conversion when any byte of its 
address space is written. The results can be accessed when any bytes of its address space is read.

\subsubsection{Memory}

Two Dallas Semiconductor DS1225AD-85 NVRAMs are used to act as both the program space and temporary memory space in this design. This is advantageous over using a pair of ROMs and a pair of static RAMs in that the NVRAMs can be programmed using the 68000 emulator and that the board space required for the larger NVRAMs is smaller. These NVRAMs also have an $85 \mathrm{~ns}$ read and write time, which is optimal for the $8 \mathrm{MHz}$ system clock (125ns period) driving the 68000 , because no additional timing circuitry is required to delay the $68000 \mathrm{read} / \mathrm{write}$ cycles.
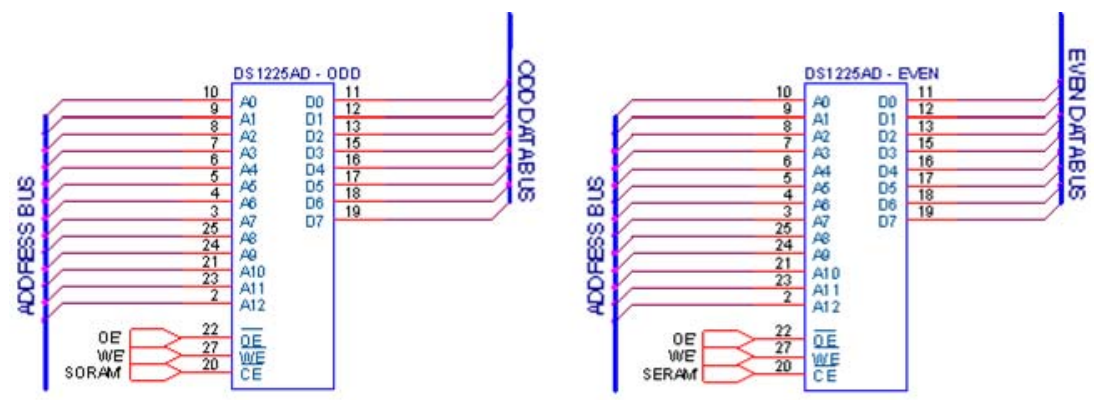

Figure 3.2: (a) Even NVRAM and (b) Odd NVRAM and connections.

Address lines A1-A13 on the 68000 (now referred to as AB1-AB13) are connected to A0-A12 on each of the NVRAMs. Data lines D0-D15 on the 68000 (now referred to as DB0-DB15) are connected to D0-D7 on the memories depending on the address space for each memory. The memory occupying odd addresses is connected to the lower byte of the data bus (DB0-DB7) while the memory occupying even addresses is connected to the upper data bus (DB8-DB15). The data lines are connected in this fashion because the memory is interleaved to make use of the data strobes (UDS' and 
LDS') on the 68000 , thereby allowing 16-bit executing from 8-bit devices. The control signals OE' and WE' are respectively connected to the corresponding NVRAM pins 22 and 27. SERAM' and SORAM' from the MMU are respectively connected to the even and odd NVRAM chip enables (CE') at pin 20.

\subsubsection{Data Transmission Acknowledge (DTACK') Signal}

An external device asserts DTACK' to signal the 68000 that data has been placed on the data bus by the device during a read cycle or that data has been read by the device from the data bus during a write cycle. Generally, the 68000 takes four clock cycles to perform a byte- or word-length read or write operation. In the event that the memory or another external device requires more time, DTACK' is used to delay 68000 instruction execution until the devices' timing requirements are met. Hence, the logic used to implement DTACK' is often referred to as a timer. DTACK' is also used to identify a fully-vectored interrupt request during an interrupt acknowledgment; however, fullyvectored interrupts are not implemented in this design.

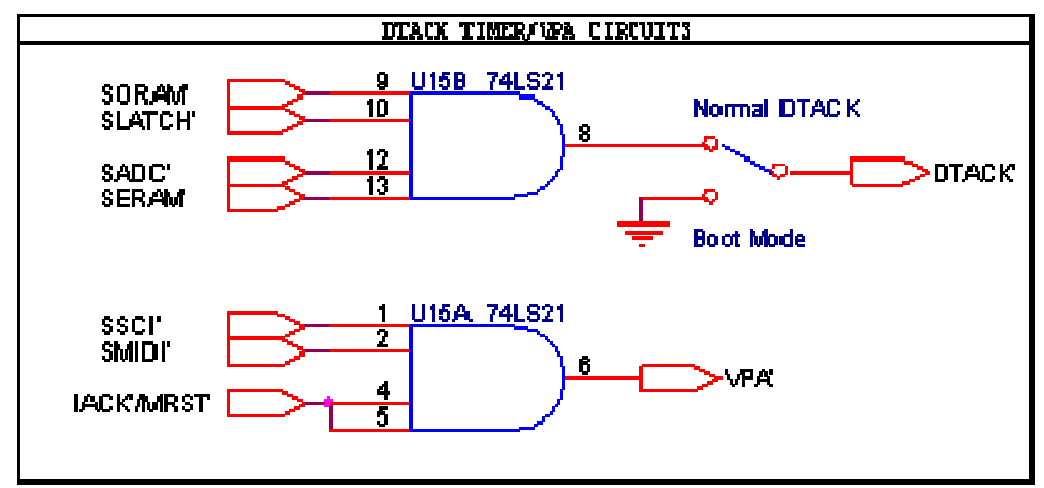

Figure 3.3: Data transfer acknowledge/valid peripheral address circuitry.

In this design, DTACK' is created by ANDing the active low chip select lines connected to the NVRAMs (SERAM' and SORAM'), A/D converter (SADC') and 
register (SLATCH'). Each chip select on each device is active-low, therefore, ANDing all the chip select inputs will cause a logic low to be produced whenever any of the previous four devices is selected. As previously mentioned, some designs require that a timer be implemented to operate DTACK', especially when interfacing to a slow memory, such as an EEPROM without a DTACK' output. Such a timer is not required here because the NVRAMs are rated for an $85 \mathrm{~ns}$ read/write cycle which is faster than the period of one $8 \mathrm{MHz}$ clock cycle (125ns).

\subsubsection{Valid Peripheral Address (VPA') Signal}

The VPA' signal is used by the 68000 to interface to the older 6800 series peripherals, such as the Motorola 6850 universal asynchronous receiver/transmitter (UART). VPA' is also used to signal the 68000 that an auto-vectored interrupt is occurring. Both VPA' functions are utilized in this design. However, there are two devices that can generate the VPA' signal for normal operation and a third device indicating the auto-vectored interrupt.

The two devices using the normal functionality of VPA' are the two Motorola M6850s (ACIAs). One of these is used for communication to the synthesizer and the other for terminal communications. The third device is the 8-bit binary down counter, which indirectly asserts VPA' when an interrupt is acknowledged (Section 3.1.11) through the IACK'/MRST' signal. The chip-selects for the two UARTS (SSCI' and SMIDI') and IACK'/MRST' are ANDed to create VPA'. 


\subsubsection{Bar Graph LED Package and Output Data Register (74LS273)}

The 74LS273 8-bit register with clear is used to illuminate the bar graph LEDs. The register is placed on the lower data bus (DB0-DB7) and occupies all odd addresses in the range of $\$ 4000-7 \mathrm{FFF}$, inclusive. Data lines DB0-DB7 are connected to register data inputs D1-D8, respectively. This particular register does not have an output or chip enable, so a rudimentary chip enable is constructed by NORing the SLATCH' and (8MHz) CLK signals. When SLATCH' is asserted, the inverted CLK signal is allowed to propagate through the NOR gate and serve as a clock to the register on pin 11 . When the clock pulses, the data on pins D1-D8 is latched and output on Q1-Q8. The RESET' signal is connected to CLR' on pin 1.

The output pins, Q1-Q8 are connected in series via a current limiting resistor to the cathode of the bar graph LEDs, which are configured in a common anode configuration. The anodes of the LEDs are connected to $\mathrm{V}_{\mathrm{CC}}(5 \mathrm{~V})$. This configuration is used because the register can sink more current on an output low than it can source on an output high. An LED will be illuminated when one of the output pins are driven low.

The bar-graph LED package actually contains ten LEDs. Since only eight LEDs are used with the register, the remaining two LEDs are used to indicate the device is powered. 


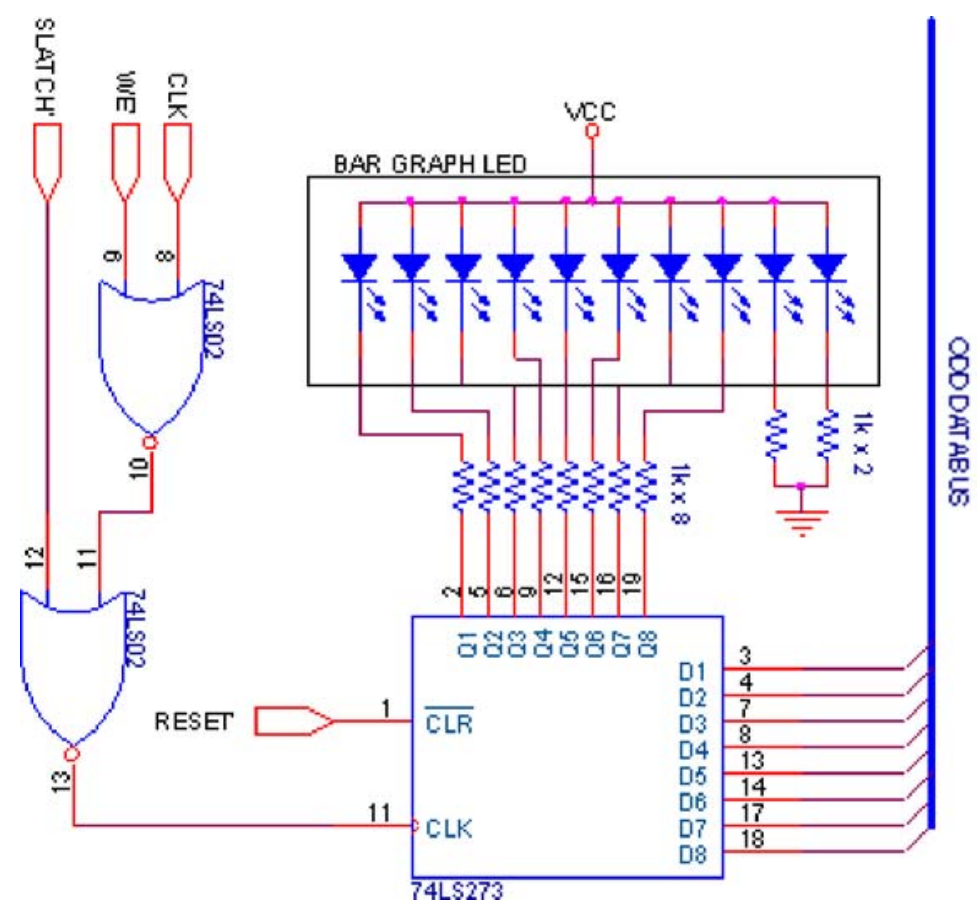

Figure 3.4: LEDs and registers.

\subsubsection{Serial Communications Interface (SCI) for Terminal Communications}

The device responsible for serial communications is the Motorola 68B50 Asynchronous Communications Interface Adapter (ACIA). This ACIA is connected to the MMU and is configured to operate when even memory locations in the range of $\$ 8000$-BFFF are accessed for read and write operations. The ACIAs have two register pairs and $\mathrm{AB} 1$ on the 68000 is used as the ACIA register select (RS) signal, selecting between the transmit/receive data and status/control registers. Since this ACIA operates on even addresses, the data lines on this ACIA are connected to the upper byte in the data bus on the 68000 . Other signals, such as the SSCI' signal from the MMU and the negated VMA' signal (VMA) from the 68000 are respectively connected to CS2' and CS1 on this ACIA; VMA is also connected to CS0. The read/write (R/W') line on the 68000 is connected directly to the read/write line on the ACIA. This allows the 68000 to 
select between the status/receive and control/transmit registers. The E-clock (EN) from the 68000 is connected to E on the ACIA to synchronize operations with the 68000 . The clear to send (CTS') and data carrier detect (DCD') inputs are unused and tied to ground. The interrupt request (IRQ') and request to send (RTS') outputs are unused are disconnected.

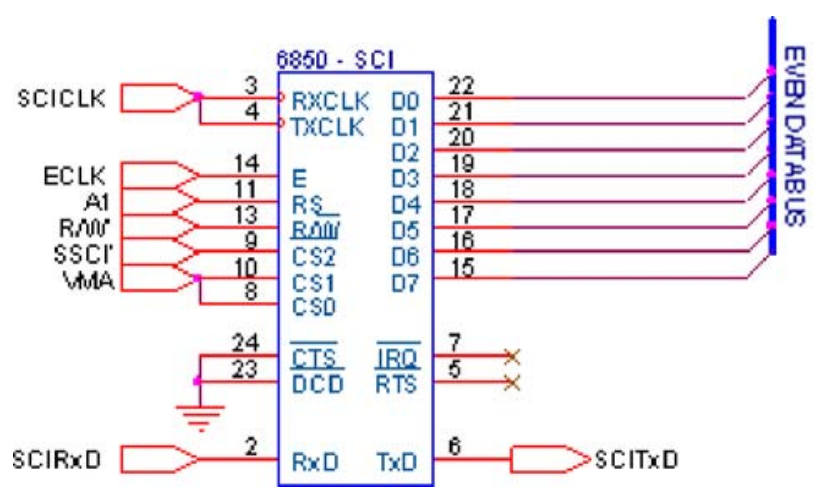

Figure 3.5: SCI asynchronous communications interface adapter.

The ACIA also requires a bit-rate generator to act as a clocking mechanism for shifting data in and out serially. The bit-rate generator (MC14411) takes a standard crystal oscillator wired in parallel with a $15 \mathrm{M} \Omega$ resistor across the inputs $\mathrm{X} 1$ and $\mathrm{X} 2$. Different frequency divisions are output on F1-F16. The different frequencies are generated with various divide-by ratios (prescalers). The primary prescaler is set by asserting or negating RSA and/or RSB; both are tied high via a $4.7 \mathrm{k} \Omega$ resistor in this design. The input frequency from the crystal oscillator to the bit rate generator is 1.8432 $\mathrm{MHz}$ and the final output is $614.4 \mathrm{kHz}$ on the $\mathrm{F} 1$ output. This output is connected to this ACIA's RXCLK and TXCLK inputs. $614.4 \mathrm{kHz}$ is used because the ACIA is set to divide the input clock frequency by 16 to prevent framing errors; this sets the transmit/receive bandwidth to 38400 bits/second. The bit-rate generator is disabled 
during the 68000 reset cycle which is realized by connecting RST' to the main reset signal (RESET'). All other outputs (F2-F16) are not connected.

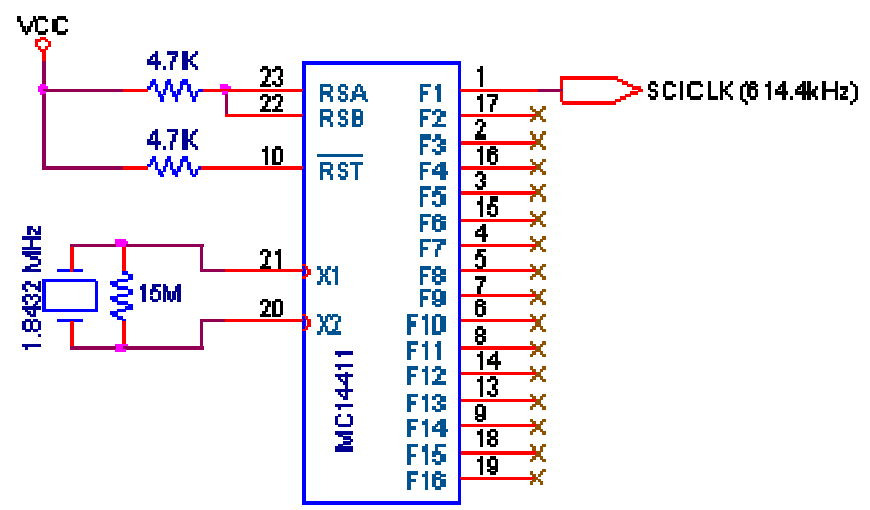

Figure 3.6: Bit-rate generator.

This ACIA also requires a voltage level shifter (MAX232) to shift the incoming RS232 voltages to standard TTL voltage levels, and vice versa for outgoing TTL signals. This device is connected per the revised specifications and diagrams provided by Maxim and can be seen in Figure 3.7. The external capacitors are used to drive a charge pump responsible for the voltage level conversion. The RS232 cable transmit (TxD) and receive $(\mathrm{RxD})$ lines are connected to R1IN and T1OUT' respectively on the MAX232. The ACIA's TTL transmit (SCITxD) and receive (SCIRxD) lines are connected to T1IN and R1OUT' on the MAX232. The remaining inputs/outputs are left disconnected.

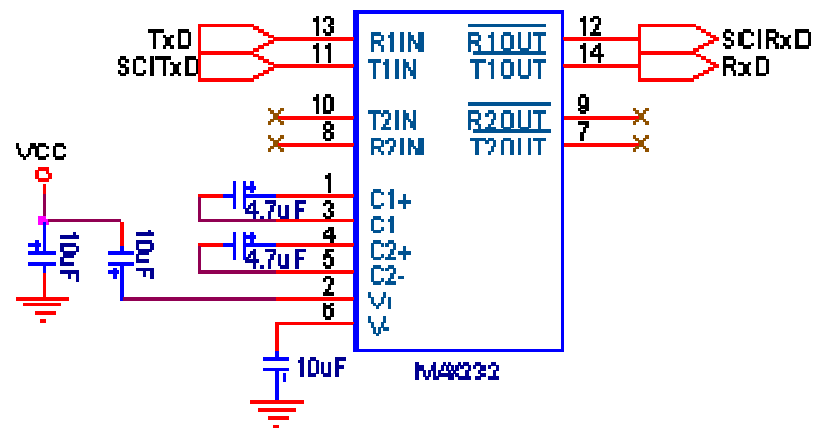

Figure 3.7: Voltage level shifter. 


\section{$\underline{3.1 .8}$ MIDI Interface}

The MIDI protocol uses the RS232 protocol at a bandwidth 31250 bps for communications. Therefore an extra ACIA is used for MIDI communications. The MIDI ACIA is connected to the lower data bus and occupies odd addresses in the range of \$8000-BFFF. AB1, CS0, CS1, RTS', IRQ', DCD', CTS' and E are connected in the same manner as the SCI ACIA. CS2' is connected to SMIDI' from the MMU. RxD is connected to MIDI in and TxD is connected to MIDI out on the MIDI cable.

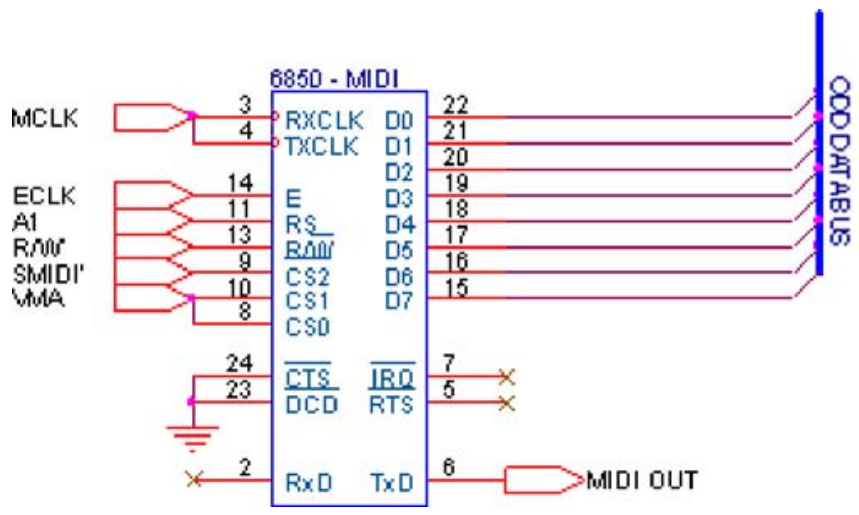

Figure 3.8: MIDI asynchronous communications interface adapter.

The TxCLK and RxCLK inputs receive a square wave at $500 \mathrm{kHz}$, as this ACIA is also configured to divide the incoming clock frequency by 16. A 74LS393 dual 4-bit counter, shown in Figure 3.9, is used to generate the $500 \mathrm{kHz}$ signal by connecting the $\mathrm{Q}_{\mathrm{D}}$ to MCLK. $\mathrm{Q}_{\mathrm{D}}$ is also connected to $\mathrm{Q}_{\mathrm{A}}$ of the second counter in case the extra frequency divisions are necessary. This would be true if the system clock were increased from $8 \mathrm{MHz}$ to $16 \mathrm{MHz}$. The active high clear signals on both counters are connected to ground so that the counters will operate continuously. All other outputs are left disconnected. 


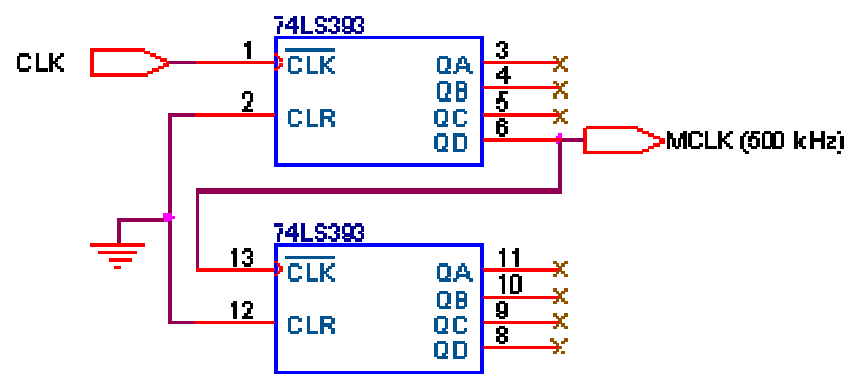

Figure 3.9: MIDI clock divider.

\subsubsection{Microphone Input Amplification and Filtering}

The microphone for this design is a Sony cardioid microphone. Although it outputs a strong signal when connected to an oscilloscope (see Figure 3.10), the output of the microphone will have to be amplified so the A/D converter can use the smaller amplitude signal. Figure 3.10 shows two separate live vocal samples taken from the microphone used in this design, which were obtained by directly connecting the microphone to the oscilloscope probe. The two samples were acquired from the same subject, but the figure on the left represents a raspy tone, whereas the figure on the right represents more of a pure tone. 


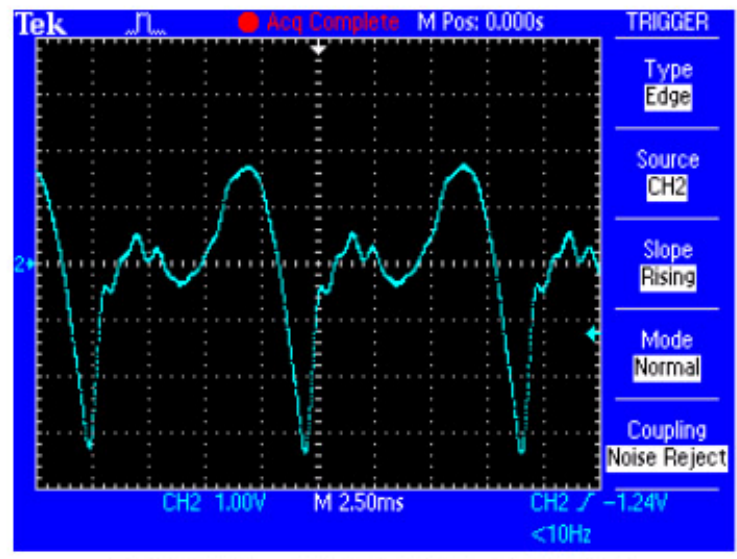

(a)

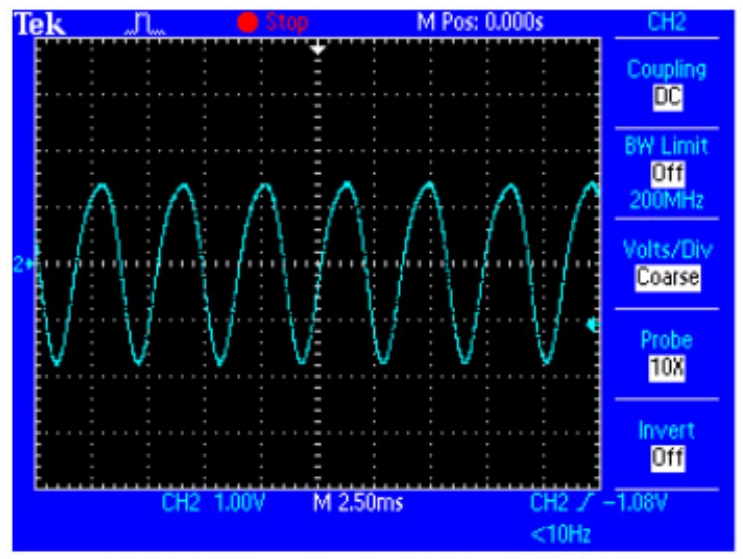

(b)

Figure 3.10: (a) Raspy vocal sample and (b) smooth vocal sample captured on a Tektronix TDS 2024 Oscilloscope.

The amplifier chosen for this design is the LM386 audio amplifier, with operation frequency range from $20 \mathrm{~Hz}-10 \mathrm{kHz}$. This was chosen primarily because it is designed for audio applications and that the power supply voltage runs at $+5 \mathrm{~V}$. A simple amplification arrangement is made by connecting the amplifier output to the noninverting terminal through a $15 \mathrm{k} \Omega$ potentiometer (R2), which is the connected to the input from the microphone through a $1 \mathrm{k} \Omega$ resistor $(\mathrm{R} 1)$. This arrangement amplifies the input signal by $1+\frac{R_{2}}{R_{1}}$, and allows the user to adjust the amplification settings. The noninverting terminal is connected to ground, and the other pins are left disconnected. 


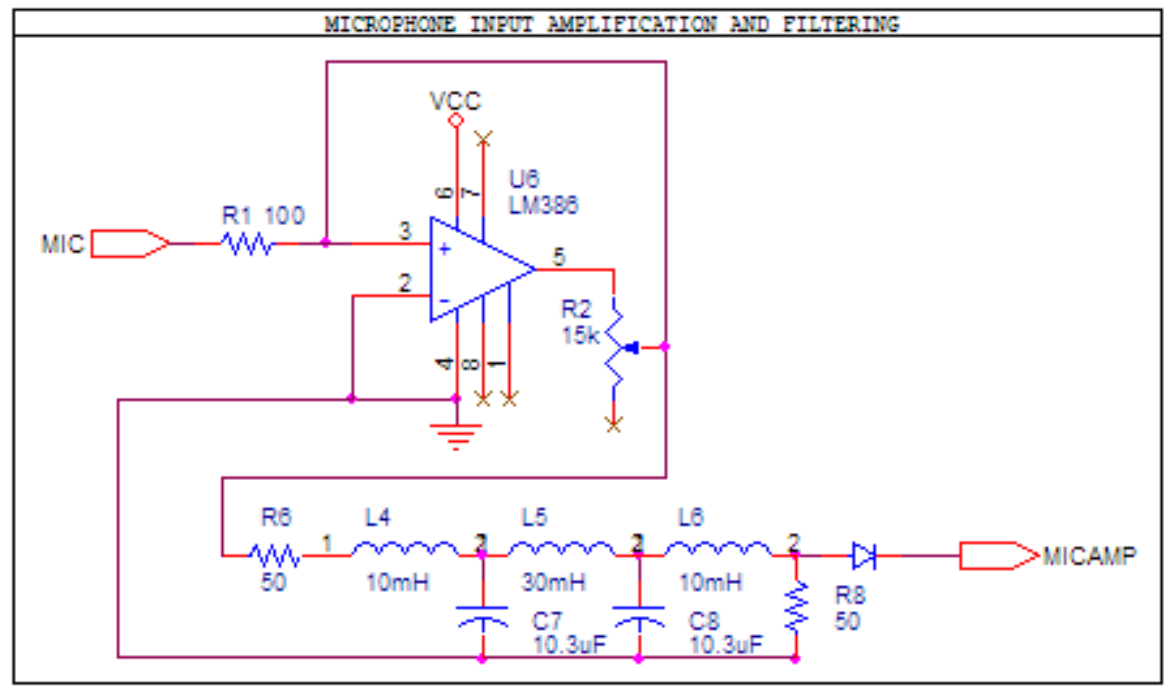

Figure 3.11: Amplification and filtering stages.

One of the problems associated with pitch identification is removing the additional harmonics associated with the fundamental frequency. Figure 2.4 illustrates this well. Therefore, filtering will be implemented on the hardware to remove some of the additional harmonics above a certain frequency. A low-pass or band-pass filter could be used for this application, however, the low-pass filter will be used because the lowerend of the spectrum is close to $0 \mathrm{~Hz}$, and less hardware will be required for implementation.

The Butterworth low-pass (maximally flat) approximation is used because it yields the best fit to an ideal low-pass filter at the lower end of the pass-band. However, the tradeoff is that the difference between the approximation and the ideal filter increases greatly toward the high end of the pass-band [Budak 1991]. The cutoff frequency for this design is set at $500 \mathrm{~Hz}$ to accommodate the hardware limitations imposed by the 68000 (see Section 3.2.2).

The output of the amplifier is also connected to a $5^{\text {th }}$ order Butterworth filter, which is also connected to a $1 / 2$ wave rectifier to remove noise above $500 \mathrm{~Hz}$ and also to 
protect the $\mathrm{A} / \mathrm{D}$ converter. The filter design was taken from the online design utility at http://www-users.cs.york.ac.uk/ fisher/lcfilter/. However, the filter with the suggested components was simulated using Spice (see Section 5.3.8 for more details) before continuing with the design. The simulations proved successful and the design was accepted. The final output of this filter is referred to as MICAMP on the schematic and is the input to the $\mathrm{A} / \mathrm{D}$ converter.

\subsubsection{A/D Converter (ADC0809) and Sampling Frequency Generator}

The $\mathrm{A} / \mathrm{D}$ converter is the device responsible for sampling the amplified vocal signal and converting the sample to an 8-bit number and resides in even memory addresses in the range $\$ 4000-7 \mathrm{FFF}$. The sampling occurs when the 68000 engages a write cycle to the A/D converter, which asserts the STARTADC signal. The sampling rate is determined by a 74HC40103 8-bit binary down counter and the interrupt routine on the 68000 .

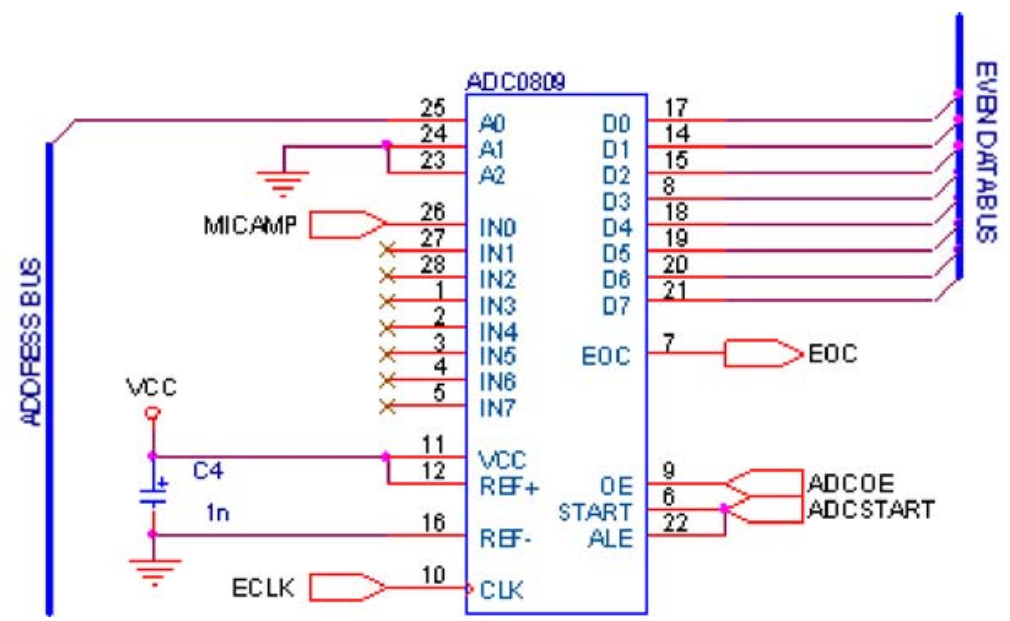

Figure 3.12: A/D converter and connections.

The A/D converter is capable of sampling eight different signals. Only one channel (IN0) is used to sample the amplified and filtered microphone signal (MICAMP); the other channels (IN1-IN7) are left disconnected. The lowest bit of the address bus 
(AB1) is connected to $\mathrm{A} 0$ of the $\mathrm{A} / \mathrm{D}$ converter. The other two lines (A1-A2) of the $\mathrm{A} / \mathrm{D}$ converter are grounded to save wiring space since only one $\mathrm{A} / \mathrm{D}$ channel is used. The data lines are connected to the upper data bus (DB8-15) on the 68000, so the device occupies even addresses in memory. The A/D converter lacks a chip enable signal, so the ADCOE signal from the MMU is used to indicate a read cycle from the data bus. The ADCSTART signal from the MMU is used during a write cycle, and signals the A/D converter to take a sample. The A/D converter reference voltages $\mathrm{REF}+$ and $\mathrm{REF}-$ are respectively connected to $\mathrm{V}_{\mathrm{CC}}(5 \mathrm{~V})$ and ground.

The $\mathrm{A} / \mathrm{D}$ converter conversion rate is determined by the ECLK signal $(800 \mathrm{kHz})$ and is not to be confused with the sampling frequency used by this design. This frequency happened to be in the middle of the acceptable frequency range and requires no additional conditioning.

The next device of interest is the adjustable sampling frequency generator as seen in Figure 3.14. This generator is created using a 74HC40103 8-bit down-counter. The counter uses the ICLK signal, which is the ECLK divided by 16 (see figure 3.13), to count down from $49_{\mathrm{dec}}-0_{\mathrm{dec}}(50$ cycles) when $\$ 31$ is input on the preload inputs. The preload inputs are tied to ground via eight $4.7 \mathrm{k} \Omega$ resistors. The divide-by number can be set by entering the new number in binary via an 8-bit DIP switch. When the switches are left open, the logic low value is input on the preload inputs, else they connect $5 \mathrm{~V}$ to the preload inputs. Once the counter counts down to $0_{\mathrm{dec}}, \mathrm{TC}$ ' is toggled low for one ICLK period. 


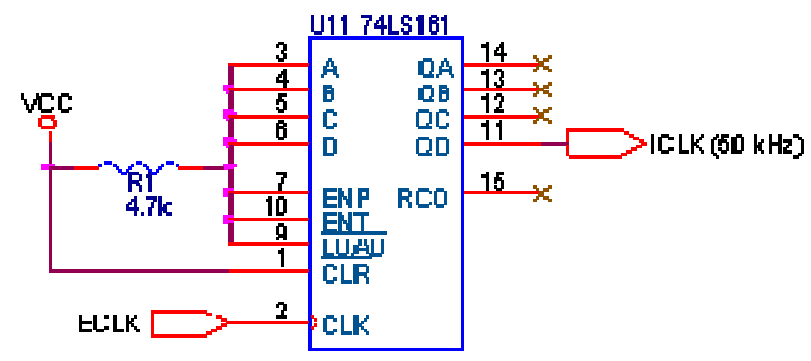

Figure 3.13: Divide-by 16 for sampling frequency generator.

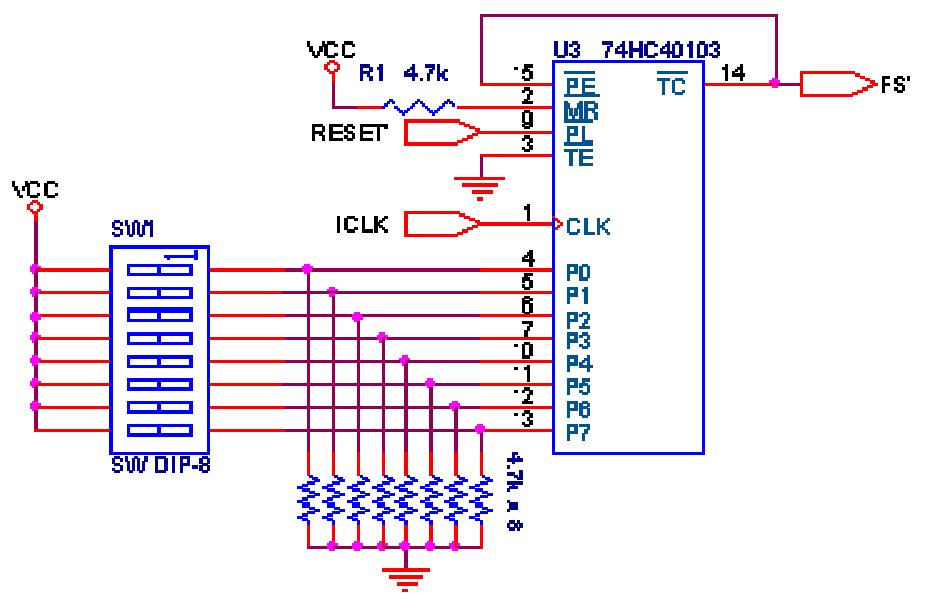

Figure 3.14: Adjustable sampling frequency generator.

TC' is renamed to FS', which is connected to IPL0' on the 68000, and PE' (synchronous preload) on the counter. This causes the sampling interrupt routine to be executed, and the value on the DIP switches to be loaded into the counter again. The asynchronous preload (PL') is connected to RESET'. Master reset (MR') on the counter is unused because the assertion of this signal causes the counter to star counting from $255_{\mathrm{dec}}$, thus it is tied high via a $4.7 \mathrm{k} \Omega$ resistor. The remaining control input, TE' is used to enable counting, and is connected to ground because sampling will be controlled in software. 


\subsubsection{A/D Interrupt Acknowledgement (IACK') and MMU Reset (MRST')}

When the 68000 receives an interrupt request on the IPLx' lines, it acknowledges the interrupt request by asserting each of FC0-FC2 simultaneously. Then, the inverted values appearing on inputs IPL0'-IPL2' are placed on AB1-AB3 to indicate the priority level of the interrupt request. The 68000 asserts address strobe (AS') and waits for the assertion of DTACK' or VPA' by the interrupting device, respectively indicating a fullyor auto-vectored interrupt request.

The primary difference between a fully- and an auto-vectored interrupt is that the auto-vectored interrupts only use the interrupt priority level in conjunction with a predetermined vector table to determine where the interrupt service routine is located. With auto-vectored interrupts, there is only one vector table entry associated with each priority level. Conversely, fully-vectored interrupts require the interrupting device to supply the vector table address (divided by four) on the lower data bus. The 68000 will take this address, multiply it by four internally and then searches for the interrupt service routine address in this vector table location. This design utilizes auto-vectored interrupts because there is only one interrupting device and the resulting hardware implementation is much simpler. Table 3.1 shows the addressing scheme for the various types of interrupts (or exceptions). 


\begin{tabular}{|c|c|c|c|c|c|}
\hline \multicolumn{2}{|c|}{ Vectors Numbers } & \multicolumn{2}{|c|}{ Address } & \multirow[b]{2}{*}{ Space 6} & \multirow[b]{2}{*}{ Assignment } \\
\hline Hex & Decimal & Dec & Hex & & \\
\hline 0 & 0 & 0 & 000 & SP & Reset: Initial SSP2 \\
\hline 1 & 1 & 4 & 004 & SP & Reset: Initial $\mathrm{PC}^{2}$ \\
\hline 2 & 2 & 8 & 008 & $\mathrm{SO}$ & Bus Error \\
\hline 3 & 3 & 12 & $\mathrm{OOC}$ & $\mathrm{SO}$ & Address Error \\
\hline 4 & 4 & 16 & 010 & SD & llegal Instruction \\
\hline 5 & 5 & 20 & 014 & $\mathrm{SO}$ & Zero Divide \\
\hline 6 & 6 & 24 & 018 & $\mathrm{SO}$ & CHK Instruction \\
\hline 7 & 7 & 28 & $01 \mathrm{C}$ & $\mathrm{SO}$ & TRAPV Instruction \\
\hline 8 & 8 & 32 & 020 & SO & Privilege Violation \\
\hline 9 & 9 & 36 & 024 & $\mathrm{SO}$ & Trace \\
\hline A & 10 & 40 & 028 & $\mathrm{SO}$ & Line 1010 Emulator \\
\hline $\mathrm{B}$ & 11 & 44 & $\infty \mathrm{c}$ & $\mathrm{SD}$ & Line 1111 Emulator \\
\hline $\mathrm{C}$ & $12^{1}$ & 48 & 030 & $\mathrm{SD}$ & (Unassigned, Reserved) \\
\hline $\mathrm{D}$ & $13^{1}$ & 52 & 034 & $\mathrm{SO}$ & (Unassigned, Reserved) \\
\hline$E$ & 14 & 56 & 038 & $\mathrm{SD}$ & Format Error ${ }^{5}$ \\
\hline$F$ & 15 & 60 & $03 \mathrm{C}$ & $\mathrm{SO}$ & Uninitialized Interrupt Vector \\
\hline \multirow[t]{2}{*}{$10-17$} & \multirow[t]{2}{*}{$16-23^{1}$} & 64 & 040 & $\mathrm{SD}$ & (Unassigned, Reserved) \\
\hline & & 92 & $05 \mathrm{C}$ & & - \\
\hline 18 & 24 & 96 & 060 & $\mathrm{SO}$ & Spurious Interrupt ${ }^{3}$ \\
\hline 19 & 25 & 100 & 064 & SO & Level 1 Interrupt Autovector \\
\hline $1 \mathrm{~A}$ & 26 & 104 & 068 & SO & Level 2 Interrupt Autovector \\
\hline 1B & 27 & 108 & $08 \mathrm{C}$ & SD & Level 3 Interrupt Autovector \\
\hline $1 \mathrm{C}$ & 28 & 112 & 070 & SO & Level 4 Interrupt Autovector \\
\hline 1D & 29 & 116 & 074 & SO & Level 5 Interrupt Autovector \\
\hline $1 \mathrm{E}$ & 30 & 120 & 078 & SO & Level 6 Interrupt Autovector \\
\hline $1 \mathrm{~F}$ & 31 & 124 & $07 \mathrm{C}$ & $\mathrm{SO}$ & Level 7 Interrupt Autovector \\
\hline \multirow[t]{2}{*}{$20-2 F$} & \multirow[t]{2}{*}{$32-47$} & 128 & 080 & $\mathrm{SO}$ & TRAP Instruction Vectors 4 \\
\hline & & 188 & $\mathrm{OBC}$ & & - \\
\hline \multirow[t]{2}{*}{$30-3 F$} & \multirow[t]{2}{*}{$48-63^{1}$} & 192 & $0 C 0$ & SO & (Unassigned, Reserved) \\
\hline & & 255 & OFF & & - \\
\hline \multirow[t]{2}{*}{ 40-FF } & \multirow[t]{2}{*}{$64-255$} & 256 & 100 & $\mathrm{SO}$ & User Interrupt Vectors \\
\hline & & 1020 & $3 \mathrm{FC}$ & & - \\
\hline
\end{tabular}

Table 3.1: Exception vector assignments. Taken from [Motorola 1993].

In this design, IPL0' is the only signal permitted to change, indicating a level interrupt request. This interrupt is auto-vectored and it is not necessary to distinguish it as such to the interrupting hardware, which greatly simplifies the circuitry needed to create the interrupt acknowledge signal (IACK').

Based on the interrupt acknowledge sequence described above, IACK' is formed by NANDing FC0-FC2 and the negated AS' (AS). IACK' is then ANDed with RESET' to form IACK'/MRST'. RESET' is included here to provide a means to disable the 
MMU during reset, because all other MMU control inputs are used. IACK'/MRST' is connected to the VPA' circuitry, to indicate an auto-vectored interrupt has occurred after the $\mathrm{A} / \mathrm{D}$ converter finishes its conversion.

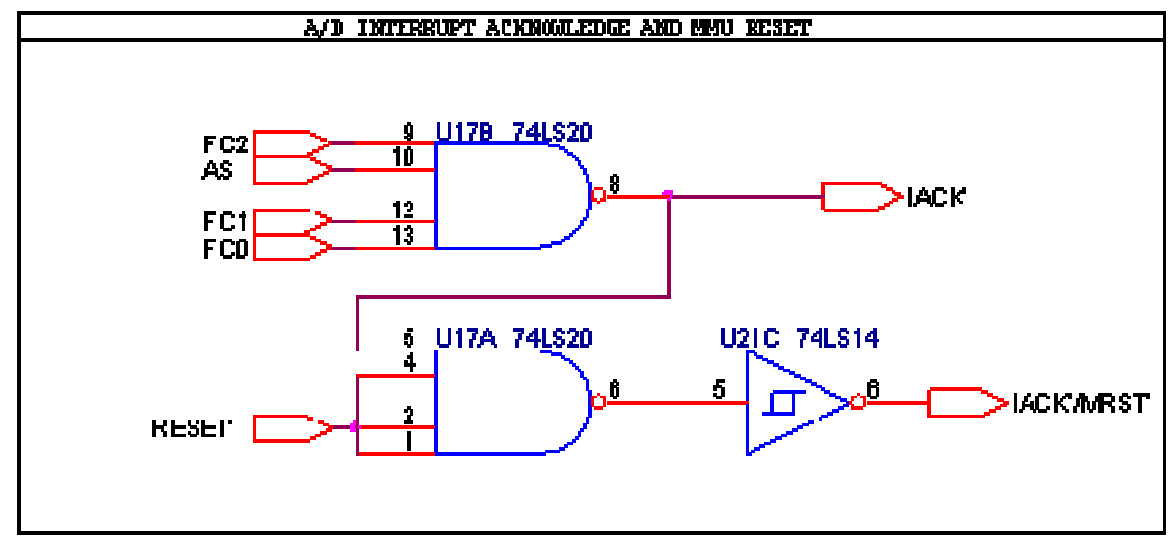

Figure 3.15: Interrupt acknowledge and MMU reset circuitry.

Originally FC0-FC2, AB1, and the end of conversion (EOC) flag from the A/D converter were to be ANDed to form IACK'. This would have also worked because EOC would normally be asserted until the A/D converter starts the conversion, at which time EOC would be negated. By the time the 68000 would start the interrupt acknowledge cycle, EOC would still be negated. Then when EOC was would be reasserted, IACK' would be asserted. This design was rejected because the 68000 would have to wait $\sim 75 \mu$ s each time for the conversion to complete, leaving only $\sim 50 \mu$ s until the next sample, and only $400,8 \mathrm{MHz}$ clock cycles to perform the ADMF calculations.

\section{$\underline{\text { 3.1.12 Power-On Reset Circuit }}$}

This hardware design has the added feature of a power-on reset circuit. This feature has been added so that when power is applied to the board, the user will not have to press a reset switch to initialize the 68000 . The power-on reset circuit is a simple circuit constructed using a simple RC filter with a diode connected in parallel to the 
resistor, with the cathode connected to $\mathrm{V}_{\text {in }}$, and the anode connected to $\mathrm{V}_{\text {out }}$. $\mathrm{V}_{\text {out }}$ is also connected to two Schmitt inverters to convert the slow rise time of the RC circuit to an oscillation-free step signal. The analysis and calculations for determining the resistance and capacitance are shown below.

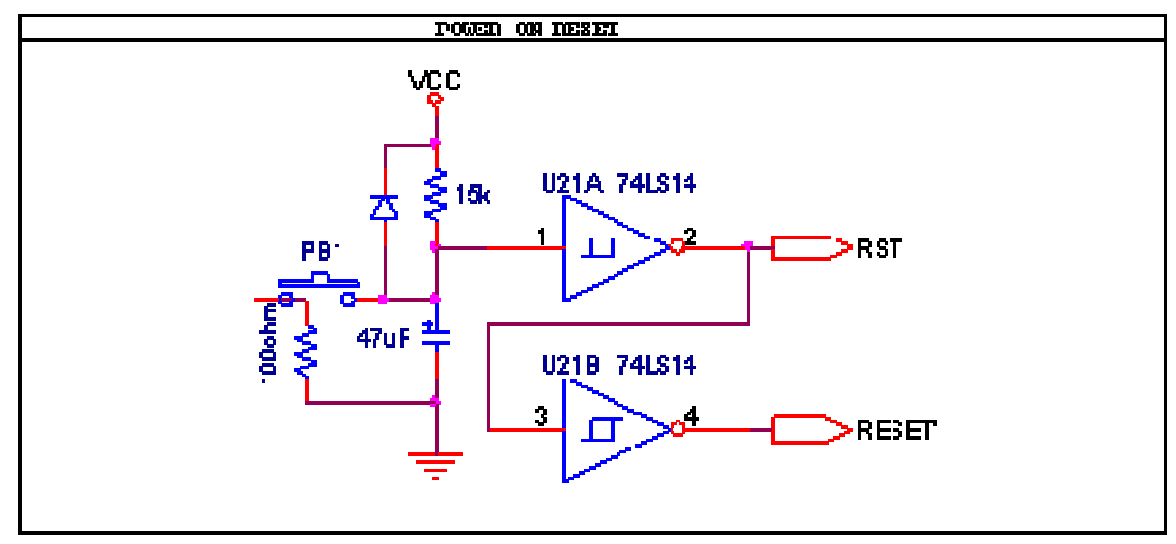

Figure 3.16: Power-on reset circuit.

The Laplace transform and the s-domain are used in this calculation since $V_{\text {in }}(t)$ is linear, time invariant, causal, and memory-less.

$$
H(s)=\frac{V_{\text {out }}(s)}{V_{\text {in }}(s)}=\frac{1}{s \cdot C \cdot\left(R+\frac{1}{s \cdot C}\right)}=\frac{1}{s \cdot R \cdot C+1}=\frac{1}{R \cdot C \cdot\left(s+\frac{1}{R \cdot C}\right)}
$$

The inverse Laplace transform is applied to the transfer function $\mathrm{H}(\mathrm{s})$ to find the impulse response of the system $h(t)$.

$$
h(t)=\frac{e^{-t /(R \cdot C)}}{R \cdot C}
$$

The input to the system is a step function with amplitude equal to the final desired voltage $\left(\mathrm{V}_{\mathrm{F}}\right)$ across the capacitor. Therefore, convolution can be used to find the voltage across the capacitor as a function of time.

$$
V_{\text {out }}(t)=h(t) * V_{\text {in }}(t)=\frac{e^{-t /(R \cdot C)}}{R \cdot C} * V_{F} \cdot u(t)=\frac{V_{F}}{R \cdot C} \int_{0}^{t} e^{-\lambda(R \cdot C)} d \lambda=-\left.V_{F} \cdot e^{-\lambda /(R \cdot C)}\right|_{\lambda=0} ^{t}=V_{F} \cdot\left(1-e^{-t /(R \cdot C)}\right)
$$


According to the Schmitt triggered inverter specifications for the 74LS14 provided by Texas Instruments, the minimum input voltage for the trigger to turn to the on state is $1.5 \mathrm{~V}$. Setting $\mathrm{V}_{\mathrm{F}}$ equal to $5 \mathrm{~V}$, the capacitance (C) can be solved for in terms of resistance $(\mathrm{R})$ and time $(\mathrm{t})$. Equations 3.3-3.6 illustrate these steps.

$$
\begin{gathered}
V_{\text {out }}=1.5=V_{F} \cdot\left(1-e^{-t /(R \cdot C)}\right) \\
1-\frac{1.5}{V_{F}}=\frac{7}{10}=e^{-t /(R \cdot C)} \\
\frac{t}{R \cdot C}=-\ln \left(\frac{7}{10}\right) \\
C=-\frac{t}{R \cdot \ln (0.7)}
\end{gathered}
$$

The hold time for the 68000 to go into reset is $100 \mathrm{~ms}$. A time of $250 \mathrm{~ms}$ is chosen to allow enough time for the 68000 to go into and remain in reset, and to allow for part tolerances and adjusting part values to match the industrial standards. A resistance of $15 \mathrm{k} \Omega$ is chosen so that the capacitor will have a smaller value. The values for $\mathrm{R}$ and $\mathrm{t}$ are substituted into Equation 3.6; solving for $\mathrm{C}$ in Equation 3.7 yields $46.73 \mu \mathrm{F}$.

$$
C=-\frac{0.25}{15000 \cdot \ln (0.7)}=46.728 \mu \mathrm{F}
$$

Since 46.73 micro Farads is not an industrial standard for capacitance, the capacitance is adjusted to 47 micro Farads. Solving Equation 3.7 for time (t), and substituting in $\mathrm{R}=15 \mathrm{k} \Omega$ and $\mathrm{C}=47 \mu \mathrm{F}$ yields a reset time of at least $251.5 \mathrm{~ms}$ before the Schmitt Triggers will toggle the signal back to a logic high. 


$$
t=-R \cdot C \cdot \ln (0.7)=-(15000)\left(47 \cdot 10^{-6}\right) \ln (0.7)=251.456 \mathrm{~ms}
$$

Figure 3.17 shows a screen capture of this implementation with $\mathrm{V}_{\mathrm{CC}}$ (green), RESET'/HALT' (yellow), and the voltage across the capacitor (blue). The delay between the time $\mathrm{V}_{\mathrm{CC}}$ reaches $5 \mathrm{~V}$ and the time RESET' is negates is approximately $170 \mathrm{~ms}$, which satisfies the $100 \mathrm{~ms}$ requirement imposed by the 68000 . The difference between the calculated time and the actual time is explained by the fact that the calculations assumed a unit step input, when in reality, this doesn't happen for a power supply with large capacitance. The calculations also neglect the fact that the Schmitt triggered inverter must also power up during the charging of the power supply's capacitors. This explains the "blip" on the yellow line in Figure 3.17. However, this "blip" and the error associated with the charging power supply are insufficient to cause the malfunction of the power-on reset circuit.

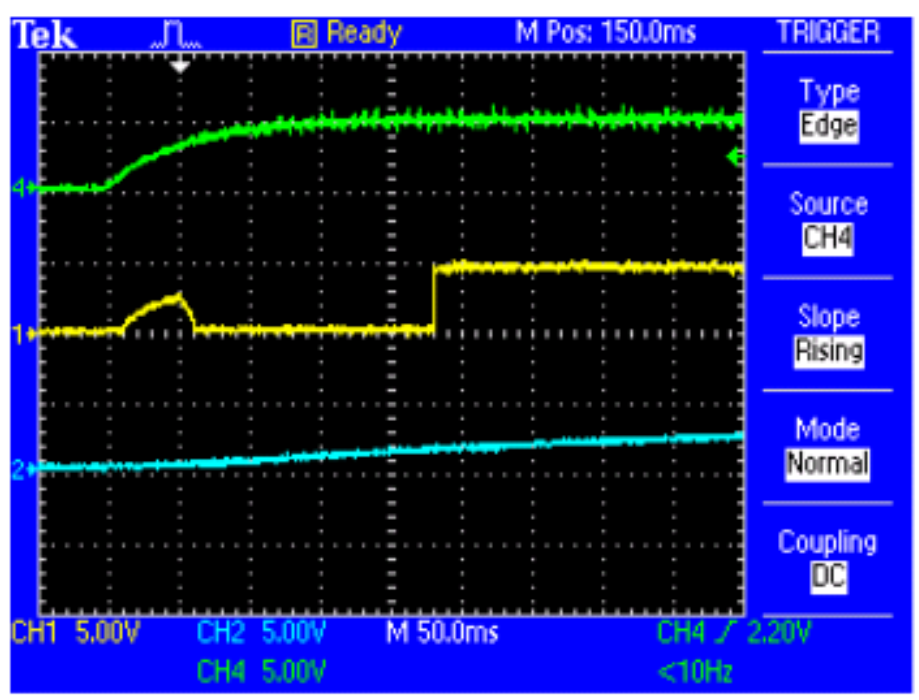

Figure 3.17: Power-on reset results.

\subsection{Software Description}


The software development for this project was completed in two stages once the general pitch detection algorithm was chosen. The first step involved developing a proof of concept in MATLAB. A variety of sound samples were input into the proof of concept and the results observed. Then the code segments were transformed into the Motorola 68000 assembly language for more simulation and eventual implementation. Section 3.2.1 describes the development of the proof of concept and the results, where as Section 3.2.2 describes the design constraints inherent to implementing the software due to the hardware design. The assembly level implementation of the supporting functions is discussed thoroughly in Section 4.2.

\subsubsection{AMDF Proof of Concept}

It felt necessary to use a high-level language to verify that the AMDF would work as expected before implementing the AMDF in assembly. MATLAB was used because of the relative ease to manipulate and plot data arrays. The MATLAB simulation code is attached in Appendix II and a variety of plots denoting the success of the AMDF algorithm are included in Section 5.1 along with a more detailed discussion of the simulations. However, the most important segment of code is included below, as this is what is used to implement the AMDF in MATLAB. In this code segment, the array of samples is $\mathrm{s}()$, and the results of each iteration is stored as $\mathrm{d}(\mathrm{n})$.

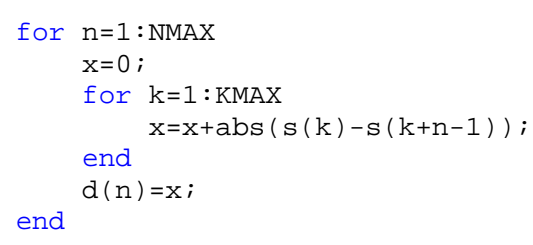

In this code segment, $\mathrm{K}_{\mathrm{MAX}}$ and $\mathrm{N}_{\mathrm{MAX}}$ are to be chosen by the developer, where $\mathrm{N}_{\mathrm{MAX}}$ represents the total number of samples to be taken and $\mathrm{K}_{\mathrm{MAX}}$ represents the size of 
the window used to identify the samples; $\mathrm{K}_{\mathrm{MAX}}$ must be less than $\mathrm{N}_{\mathrm{MAX}}$. Varying these values alters the detected fundamental frequency slightly and larger values require more computation time. Experimentation revealed that setting $\mathrm{N}_{\mathrm{MAX}}$ in the region of 100-200 and $\mathrm{K}_{\mathrm{MAX}}$ in the region of $1 / 4$ to $1 / 2$ the value of $\mathrm{N}_{\mathrm{MAX}}$ yielded good results.

\subsubsection{Implementing the Algorithm for the 68000}

After the AMDF functionality was verified using MATLAB, the next step was to implement the ADMF in assembly. A few revisions were written, mainly to reduce the number of clock cycles. However, the functionality of the final revision was tested and verified using a fixed data array with the same values in $\mathrm{s}()$ from the MATLAB simulation. The routines were simulated using the EASy68K editor, assembler, and simulator and the results can be found in Section 5.2.

Originally, the hardware design revolved around an $8 \mathrm{MHz}$ system clock. It will be shown later that this clock will be insufficient unless changes to the design are made. However, the preliminary discussion of the software will include timings for an $8 \mathrm{MHz}$ system clock.

The primary algorithm for the AMDF consists of a pair of nested for-loops that creates a computational complexity of $\mathrm{O}\left(\mathrm{K}_{\mathrm{MAX}} * \mathrm{~N}_{\mathrm{MAX}}\right)$. If $\mathrm{K}_{\mathrm{MAX}}=96$ and $\mathrm{N}_{\mathrm{MAX}}=192$, this means that there will be a total of 18432 iterations through the array before considering any calculations made with the data. In reality, the loops take approximately $1.1 \mathrm{M}$ clock cycles according to the Easy68K simulator, which, at an $8.0 \mathrm{MHz}$ system clock equates to approximately 0.1375 seconds. This value includes all the steps necessary to perform the AMDF, but not to find the fundamental frequency or output the necessary commands to the synthesizer. 
With these considerations in mind, there are two approaches for an interruptdriven sampling routine. The first approach involves storing all the data from the analogto-digital converter in an array first, then performing all the calculations after a certain number of measurements have been saved. The second approach involves storing the first M-number samples, then performing the inner loop of the MATLAB code after each consecutive reading until the number of readings is equivalent to the sum of $\mathrm{M}$ and $\mathrm{N}$.

The two different approaches have different advantages and disadvantages. With the first approach, there is an abundant amount of time remaining between samples. Therefore, the processor can run at a slower clock frequency and sample at a higher frequency. The disadvantage to the first approach is that the bulk of the calculations are performed at the end of a sampling cycle, and the number of calculations required to perform the AMDF and identify the fundamental frequency requires approximately $1.2 \mathrm{M}$ clock-cycles which equates to approximately 0.15 (8 MHz clock) seconds before adding the overhead to send the MIDI information to the synthesizer. The goal, however, is to reduce the delay at less than $20-30 \mathrm{~ms}$ so as not to be audibly noticeable by the user [Lago 2004].

The second approach performs most of the calculations for the AMDF between each sampling interrupt routine. The advantage of this approach is that the remaining calculations to process the AMDF results require approximately 22000 clock cycles, which equates to approximately $2.75 \mathrm{~ms}$ ( $8 \mathrm{MHz}$ clock) before adding the overhead to send the MIDI information to the synthesizer. The disadvantage with this approach, however, is that the inner-loop requires approximately 5600 clock cycles, and if the 
sampling rate is $3520 \mathrm{~Hz}(2 \times 1760 \mathrm{~Hz})$, this leaves $\frac{8 \mathrm{MHz}}{3520 \mathrm{~Hz}}=2461$ clock cycles to perform the calculations, meaning that the routine is over budget by 3139 clock cycles.

Regardless of which approach is chosen, it is now obvious that the clock frequency must increase to accommodate the real-time calculations, or the sampling rate must significantly decrease. If the second approach is chosen, then the sampling frequency must decrease, regardless.

Later versions of the 68000 are capable of running at $12 \mathrm{MHz}$ and $16 \mathrm{MHz}$. If a $16 \mathrm{MHz}$ clock is chosen and the maximum allowed vocal frequency is reduced from $1760 \mathrm{~Hz}$ to $1250 \mathrm{~Hz}$ (sampling rate of $2500 \mathrm{~Hz}$ ), which corresponds to a difference of six chromatic steps or $1 / 2$ octave, the new sampling rate would leave 6400 clock cycles between samples to perform the inner-loop calculations. The extra clock cycles will be used to ensure the 68000 has enough time to finish all calculations during the interrupt routine and to allow for further additions to the interrupt routine for the development interface and options. Furthermore, the sampling rate of $2500 \mathrm{~Hz}$ would be easily generated by the hardware and represents a convenient integer number for division later in the routine.

The other choice is to significantly reduce the sampling frequency to $1000 \mathrm{~Hz}$ and leave the clock at $8 \mathrm{MHz}$. This leaves 8000 clock cycles between interrupt service requests to perform the calculations described above. However, this would reduce the maximum allowed frequency to $500 \mathrm{~Hz}$, which corresponds to a high note of B4, (see Table 4.2). This results in a difference of eleven chromatic steps, which is almost an entire octave below the original sampling frequency. The advantage to this choice is that the lab equipment can be used to perform real-time simulations with the hardware design 
in its entirety. Therefore, the $8 \mathrm{MHz}$ clock will used in conjunction with a lowered sampling frequency. 


\section{CHAPTER IV - SYSTEM IMPLEMENTATION}

This chapter explores the two necessary components of this design, namely the implementation of the hardware and software for the prototype. The hardware section focuses on the techniques used for construction; a detailed discussion of the design can be found in Section 3.1. The software section focuses on the implementation of the AMDF in assembly. Each functional component required to implement this algorithm in assembly will be discussed in detail.

\subsection{Hardware}

There are three methods available for assembling this hardware, each with a varying level of permanence and usability. The first method that may come to mind is bread-boarding. This method was immediately rejected because, in the lab, students typically have to reduce the typical $8 \mathrm{MHz}$ clock frequency to $4 \mathrm{MHz}$ to reduce the noise on the breadboard tracks. The next method involves laying copper tracks and sending the design to be fabricated. Once the design is received, the components would be soldered to the board. However, the final product is rather permanent and difficult to modify should there be an error in the hardware design. Therefore, the hardware for this design will be assembled using VectorBord prototyping board. The model used is double-sided and contains individual solder pads spaced 0.050 inches apart on both sides with nonplated-through holes. This method combines the advantages of both the previous methods, in that designs are somewhat permanent, but lend themselves to rework more easily than a fabricated design. The prototyping board consists of 2960 pads arranged in 40 rows by 74 columns on either side of the board, leaving a total of 5920 pads for potential use when considering using both sides of the board. 
The first necessary step to implementing the hardware design is to create a board layout. This is always done for fabricated design and sometimes done for prototyping. It is easier to solder tracks, parts, and wires when the layout has been visualized and developed on paper. In industry, there exists software for board layout; however, that software is expensive and doesn't necessarily lend itself to prototyping layout as well as track layout. Therefore, Adobe Acrobat CS is used to draw the board layout. This might not be the best tool, but Photoshop files support various image layers, which has its advantages.

To start, the prototyping board was rendered to scale by creating a series of gridlines and can be seen in Figure 4.1. The gridline for every tenth pad was made thicker for a quick visual reference. No through-holes were rendered because they clutter the display. A large blue track representing the ground track was placed around the gridlines. Finally, two yellow segments were placed at the bottom of the board to represent the area on the board where the ground track is removed. This is done so that the bottom segment of the topside ground track can be used to carry the system clock across the board without as much fear of noise permeating the neighboring lines. 


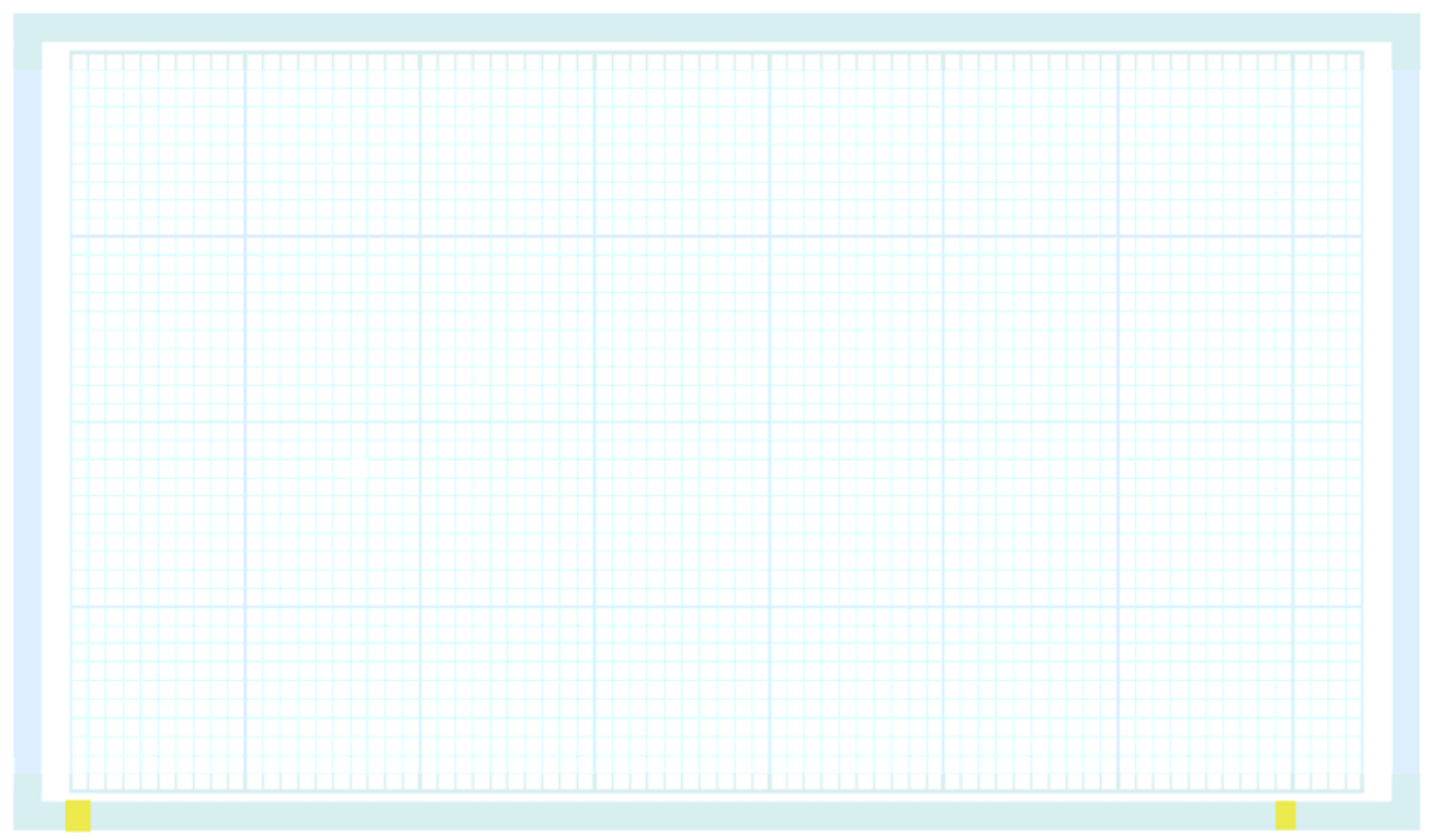

Figure 4.1: Topside view of the empty prototyping board.

Even before placing digital parts on the board, it was necessary to conceptualize the size of the parts in relation to the size of the board. The parts were loosely arranged based on their pinouts and size. Once the loose arrangement was loosely finalized, the parts' digital equivalents were placed on the digital grid as shown in Figure 4.2. The parts were created using copies of the pinouts taken from the various part specifications, primarily so the pin names would be included on the board layout and could be used for debugging and quick reference when looking at the physical creation. 


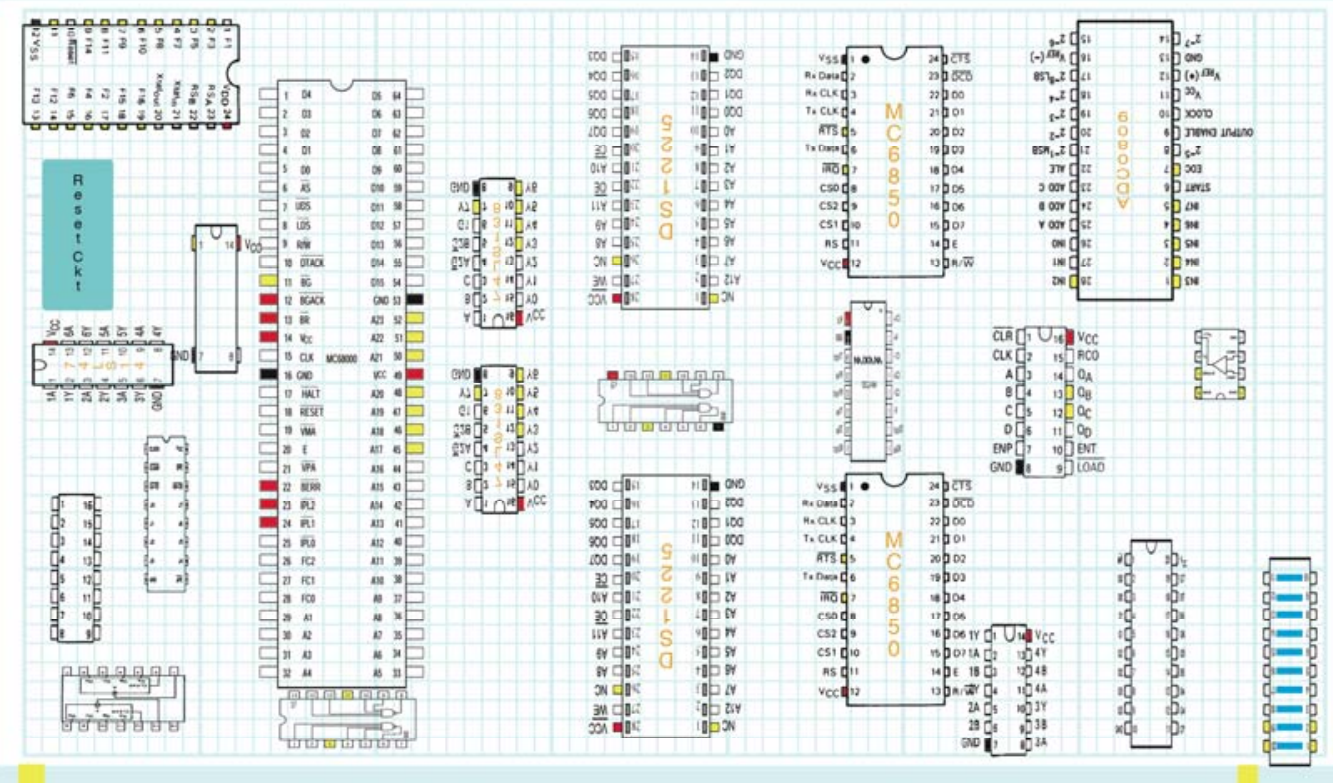

Figure 4.2: Topside view of loosely place parts on the prototyping board.

The next step was to create a convention for placing tracks, wires, and discrete components. One may also notice the additional colorings on the pinouts above. These were added to certain pins for quick reference while creating the board layout. Table 4.1 describes all conventions used in the board layout. The choices for color were mostly arbitrary.

\begin{tabular}{|c|c|c|}
\hline & Item & Color \\
\hline \multirow[b]{3}{*}{$\alpha$} & VCC & Red \\
\hline & Tied High & Red \\
\hline & VSS/GND & Black \\
\hline & Not Connected & Yellow \\
\hline & Topside Track & Blue \\
\hline & Topside Wire & Orange \\
\hline (1) & Backside Track & Green \\
\hline & Backside Wire & Purple \\
\hline & $\begin{array}{c}\text { Resistors/Caps } \\
\text { Either Side }\end{array}$ & Red \\
\hline
\end{tabular}

Table 4.1: Board layout color-coding conventions. 
Once the conventions and the loose layout were finalized, the board layout began. For this design, a combination of solder tracks and wires were used to create the layout. For reference, connecting two or more pads on the prototyping board together by dragging the solder across the thin break between the pads creates a solder track. Conversely, soldering a wire involves soldering the wire to two separate pads on the board. There is a mixture of solder tracks and wires in this design to reduce the clutter of additional wires. The final board layout for the top and bottom sides of the board can be found in Appendix I.

\subsection{Software}

Now that the clock frequency and sampling rate have been finalized and the hardware design and layout have been completed, it is appropriate to discuss the methods to identify a frequency. After the 68000 initializes and all preliminary initializations in code are complete, the general approach is to sample the data, perform the AMDF calculations, find the locations of the minima, calculate the periods between the minima, calculate the average frequency, and send the data to the synthesizer.

\section{$\underline{4.2 .1 \text { Initializations }}$}

Upon coming out of reset, the 68000 code clears the data and address registers used for the counters and resets the address registers used for the sampled data array and the AMDF results array. The two ACIAs are also reset by sending $\$ 03$ to their control registers. Then the ACIAs are configured to divide their clocks by $16_{\mathrm{dec}}$, to set the parity equal to none, to set the data for 8 data bits, and one stop bit for general communications. This results in a bit rate of $38400 \mathrm{bps}$ for the SCI ACIA and 31250bps for the MIDI 
ACIA. Next, interrupt requests are enabled on the 68000 by ANDing the status register (SR) with \$F8FF. At this point, the software loops by repeatedly calling the SENDMIDI routine and waits for an interrupt.

\subsubsection{Sampling and performing the AMDF (ADCIRQ)}

The sampling generator is the only device in this design that generates an (autovectored) interrupt. The sampling occurs every sampling period as adjusted by the adjustable sampling frequency generator (see Section 3.1.10), and this routine executes at that time. This interrupt service routine is perhaps the most important routine in this design because it is the top level. Figure 4.5 contains a flow diagram representing this routine. Please note that this figure represents one routine iteration. Additional iterations are performed on subsequent interrupt requests.

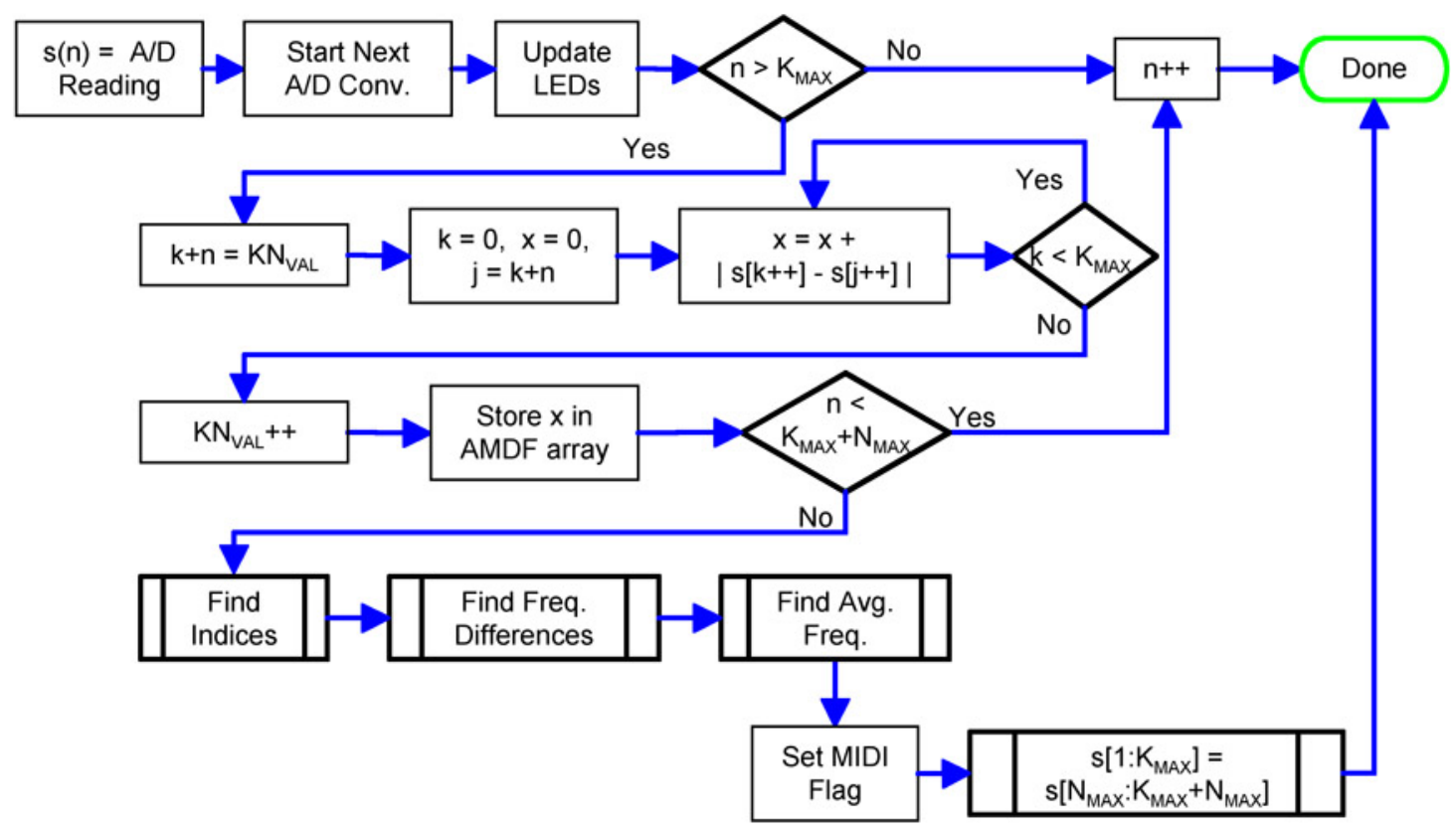

Figure 4.3: Flow diagram for interrupt service routine (ADCIRQ)

When this interrupt service routine begins execution, the data sample from the $\mathrm{A} / \mathrm{D}$ converter is read and stored. Then a value is written to the $\mathrm{A} / \mathrm{D}$ converter to start the 
next conversion. This command is placed as close to the beginning as possible because the remaining assemble commands used to calculate the AMDF array do not guarantee a fixed number of clock cycles between each execution of the routine. Therefore, placing the next write instruction immediately after the read instruction guarantees the next conversion will be complete before the next sampling period and this eliminates any sampling period discrepancy due to software timing, except the very first sample.

This service routine is also responsible for calculating the AMDF array values by executing the nested for-loops as discussed in the proof of concept section (see Section 3.2). A total of $K_{M A X}+N_{M A X}$ samples must be taken to complete the AMDF. However, at least $\mathrm{K}_{\mathrm{MAX}}$ (number of samples in the sliding window) must be taken before the innerloop calculations may occur. Thus, each time a sample is taken, the number of measurements is compared to $\mathrm{K}_{\mathrm{MAX}}$. If the number of measurements is less than $\mathrm{K}_{\mathrm{MAX}}$, the routine exits and the 68000 waits for the next sampling interrupt request to occur, else the AMDF calculations begin.

After at least $\mathrm{K}_{\mathrm{MAX}}$ samples are taken, one (inner-loop) iteration of the AMDF is calculated, and the 16-bit result is stored in a new array in memory named DD, which is equivalent to the d-array in the simulations and literature. However, this will herein be referred to as the AMDF array to avoid confusion. The data stored during each inner loop calculation is $x=x+|s(k)-s(k+n)|$, where $n$ and $k$ are indices and $s$ is the sampled data array. After $\mathrm{K}_{\mathrm{MAX}}$ elements of the single iteration through the inner loop have been processed, the interrupt routine exits. This process continues until $\mathrm{K}_{\mathrm{MAX}}+\mathrm{N}_{\mathrm{MAX}}$ samples are taken, at which time interrupt requests to the 68000 are disabled in software, and the remaining functions to find the fundamental frequency are called. 


\subsubsection{Finding the periods between minima (FINDINDICES)}

This routine searches through the ADMF results stored in the DD array and finds the corresponding AMDF array indices correlating to the local minima that fall below a defined threshold. Figure 4.6 illustrates a typical set of results from using the AMDF, the threshold value, and the identified local minima falling below the threshold. Once these array indices are identified they will be used to identify the fundamental frequency.
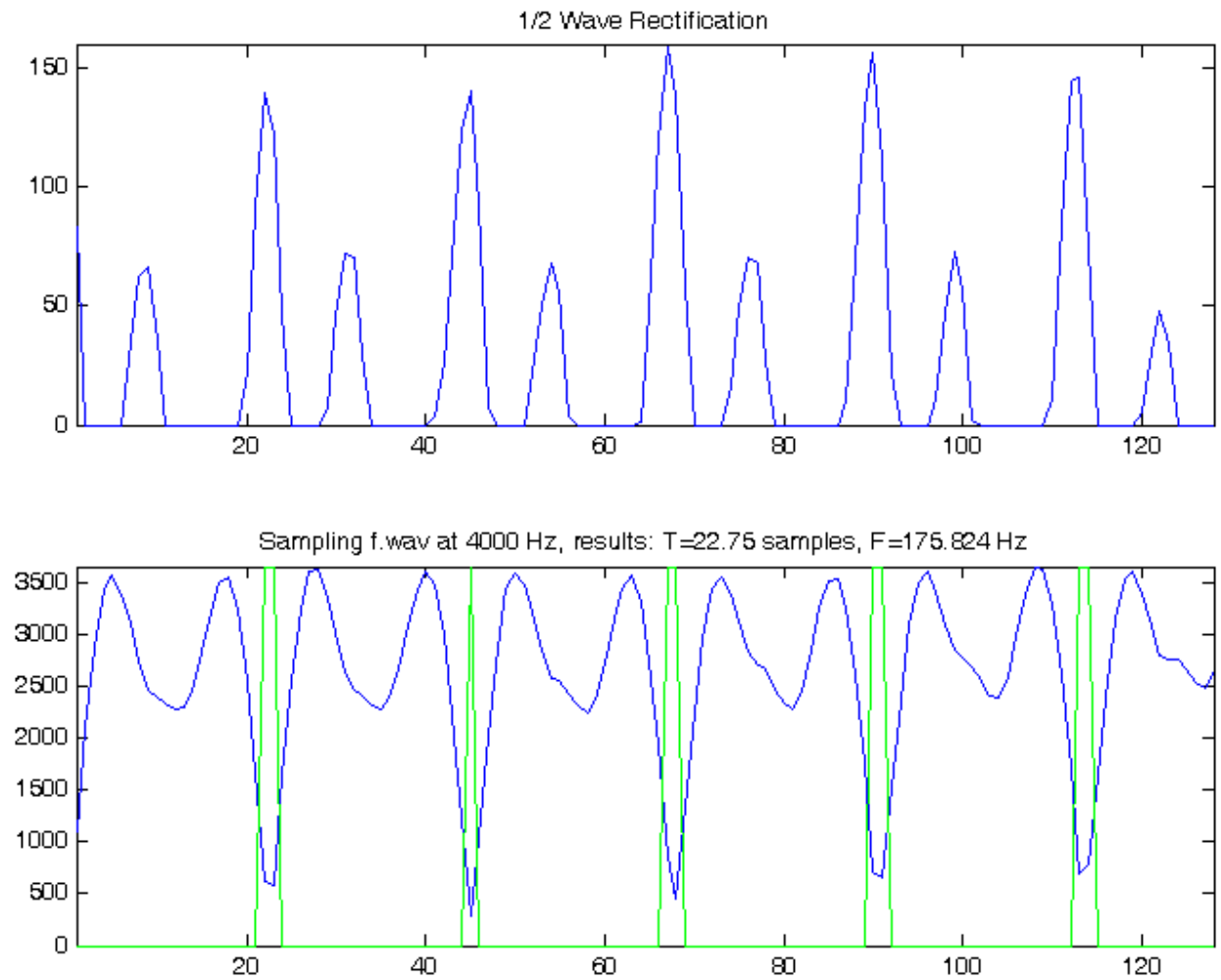

Figure 4.4: Sample AMDF results (blue) and regions where the data fall below the detection threshold (green).

The general approach to identifying the useful minima in the AMDF results array is to save all indices of minima where the AMDF samples fall below $25 \%$ of the maximum value in the AMDF array. Since there are two sets of minima each period, it is necessary to define a cutoff point for which to define a minimum. The range of the data 
will vary depending on the number of samples considered, thus it is necessary to redefine the threshold value each time the AMDF finishes (before this function executes). The threshold value is set to $25 \%$ of the maximum value in the AMDF array because setting the threshold to $25-30 \%$ resulted in better performance during the MATLAB simulations when performing a search. However, multiplying by $25 \%$ is equivalent to dividing by four, which is a simpler operation in assembly, thus requiring fewer precious clock cycles. Once the threshold has been identified, a sequential search for the local minima begins as described in the following paragraphs and graphically in Figure 4.7.

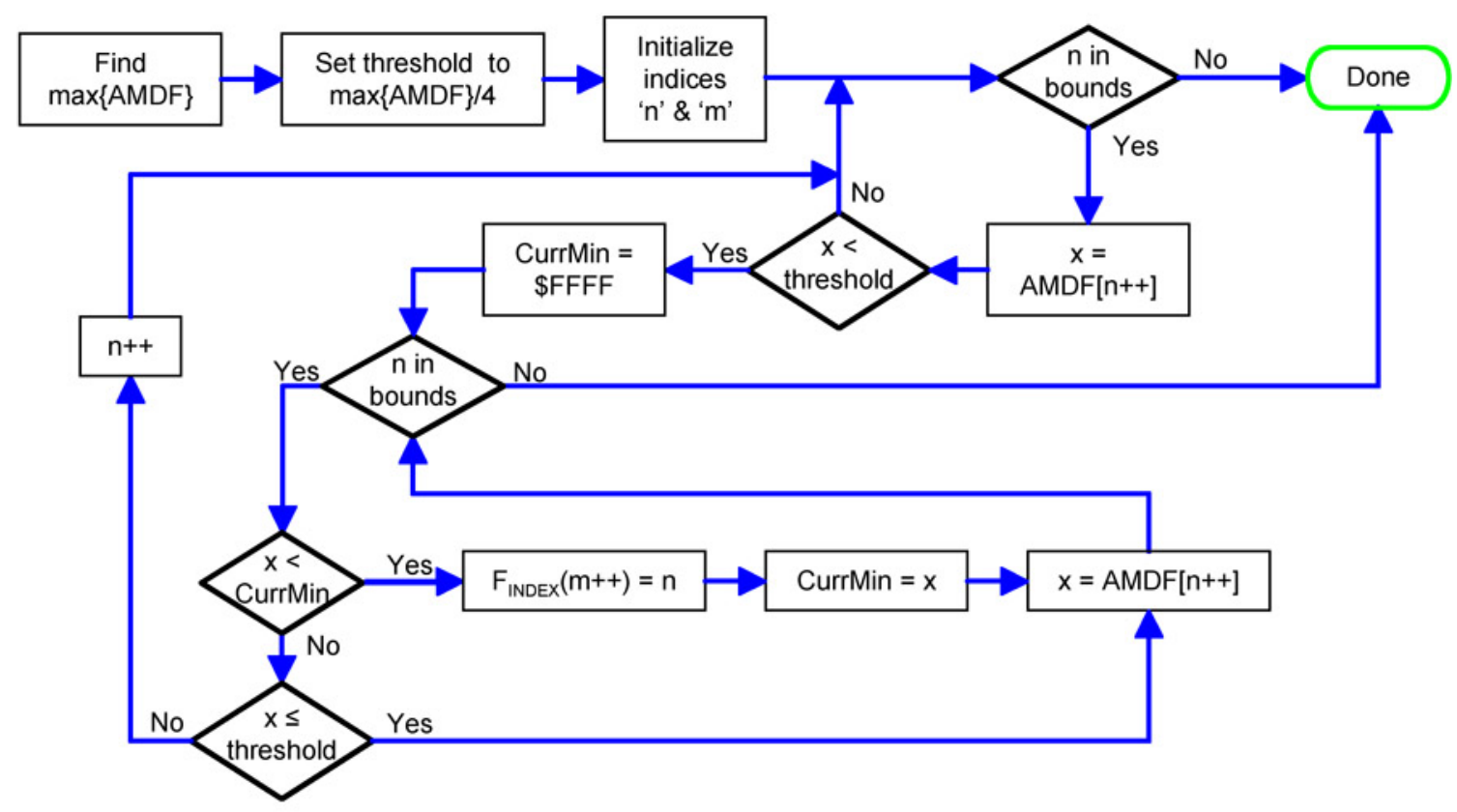

Figure 4.5: Flow diagram for finding indices.

The first step in finding the minima is to identify the maximum value in the AMDF array. A variable called MAXOFDD is initialized to zero and a sequential search through the array is executed and each value is compared to MAXOFDD. If the current value is greater than MAXOFDD, MAXOFDD assumes the current AMDF value. Once 
the search is complete, MAXOFDD is stored in a data register and divided by four to set the threshold for the next set of searches.

Then this routine loads the initial AMDF array index into an address register and iterates through the array until a value that is less than or equal to the threshold is identified. If the current value is less than the threshold, the routine branches to a smaller loop and finds the local minimum value in the data that fall below the threshold, while continuing to iterate through the AMDF array. Once the sequentially values rise above the threshold, the array index pointing to the minimum value in the aforementioned data segment is stored to the $\mathrm{F}_{\text {INDEX }}$ array. The smaller loop exits and the other loop resumes checking for values falling below the threshold. This process repeats until all values in the AMDF results array have been examined.

\subsubsection{Calculating the difference between indices (FDIFF)}

Once the $\mathrm{F}_{\text {INDEX }}$ array has been populated, the periods can be identified in units of address offsets. Subtracting the array indices will result in the number of sample periods between each minimum. This is represented by the formula: $F_{\operatorname{INDEX}}(n)=F_{\operatorname{INDEX}}(n+1)-$ $\mathrm{F}_{\text {INDEX }}(\mathrm{n})$. The $\mathrm{F}_{\text {INDEX }}$ array is re-used to save memory and because the data in its present form is not used later in the code. Figure 4.6 shows a flow diagram for this function. 


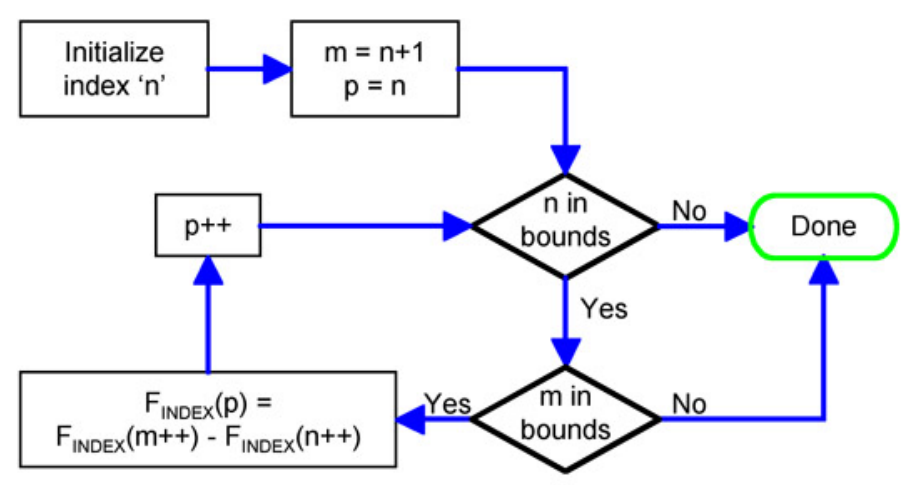

Figure 4.6: Flow diagram for finding index differences

The periods are calculated by loading the initial address of $\mathrm{F}_{\text {INDEX }}$ into two address registers. One address register is incremented by two to point to the next index in the array, thereby accessing $\mathrm{F}_{\operatorname{INDEX}}(\mathrm{n}+1)$. The other address register is used to access $\mathrm{F}_{\text {INDEX }}(\mathrm{n})$. As the array is examined, $\mathrm{F}_{\operatorname{INDEX}}(\mathrm{n}+1)$ is compared to the end-of-array flag. If equal, the routine exits, else the subtraction is stored in $\mathrm{F}_{\text {INDEX }}(\mathrm{n})$. An end-of-array flag is used instead of counting the number of entries in the array because the number of entries varies and can make debugging in a hex-dump more difficult, whereas an end-of-array flag is easy to spot at a glance. Before the routine exits, the last entry of the original $\mathrm{F}_{\text {INDEX }}$ array is replaced with a different end-of-array flag as the last entry in $\mathrm{F}_{\text {INDEX }}$ is no longer valid. This new end-of-array flag will be used by FAVG (see Section 4.2.5).

\subsubsection{Finding the fundamental frequency (FAVG)}

This routine sums all entries in $\mathrm{F}_{\text {INDEX }}$ after FDIFF has been executed and calculates the average frequency for the array of samples. Recall in Figure 4.6 that the AMDF produces a series of minima. Technically the difference between any two close minima will yield the fundamental period $\left(\mathrm{T}_{0}\right)$. The results, however, produce more than one of these minima, which will cause the associated period to vary. Hence, the average 
is used to create one fundamental period, which is converted to the equivalent fundamental frequency $\left(f_{0}\right)$.

The number of entries in $\mathrm{F}_{\text {INDEX }}$ is multiplied by the sampling rate after the sum of the periods (stored in $\mathrm{F}_{\text {INDEX) }}$ is calculated. This result is divided by the sum of elements in $F_{\text {INDEX }}$ from FDIFF. The quotient is an integer and is stored as the fundamental frequency $\left(f_{0}\right)$. Equation 4.1 mathematically represents this process.

$$
f_{0}=\frac{f_{s} \cdot N}{\sum_{i=1}^{N} F_{I N D E X_{i}}}
$$

The remainder is used to determine whether to round the result up. If the remainder is greater than or equal to half of the divisor (i.e. the sum of elements in $\mathrm{F}_{\text {INDEX }}$, then $f_{0}$ should be rounded up. This check is accomplished by dividing the previously calculated sum of all $\mathrm{F}_{\text {INDEX }}$ entries by two and by comparing the remainder from the division to this value. If the remainder is greater than or equal to the half the sum, $f_{0}$ is incremented by 1 .

\subsubsection{Converting frequency to MIDI (FREQ2MIDI)}

This routine is actually the simplest of all the routines regarding conversion of data. The binary search is one of the first methods that come to mind to convert the frequency to a MIDI note number since the numbers can be expressed as ordered lists. This method has a computational complexity proportional to $\mathrm{O}(\log \mathrm{N})$ and is memory efficient. However, execution time efficiency and consistency are of greater interest in this design. Instead of using the binary search to find the frequency in a table, interpolating between numbers, and assigning a value for the MIDI note number, the 
frequency $\left(f_{0}\right)$ itself is used as an index offset for a very long linked list, since $f_{0}$ is an integer. This linked list contains each MIDI note number $\left(36-84_{\mathrm{dec}}\right)$ corresponding to frequencies from $63-1078 \mathrm{~Hz}$ and Table 4.2 shows correlating integer frequencies to pitches and MIDI note numbers. 


\begin{tabular}{|c|c|c|c|c|}
\hline Pitch & $\begin{array}{c}\text { Actual } \\
\text { Freq. (Hz) }\end{array}$ & $\begin{array}{c}\text { Valid } \\
\text { Freqs. }(\mathrm{Hz})\end{array}$ & $\begin{array}{l}\text { Address } \\
\text { Offsets }\end{array}$ & $\begin{array}{l}\text { MIDI Note } \\
\text { Number }\end{array}$ \\
\hline $\mathrm{C} 2$ & 65.406 & $63-67$ & $0-4$ & 36 \\
\hline $\mathrm{C} \# 2$ & 69.296 & $68-71$ & $5-8$ & 37 \\
\hline D2 & 73.416 & $72-75$ & $9-12$ & 38 \\
\hline $\mathrm{D} \# 2$ & 77.782 & $76-80$ & $13-17$ & 39 \\
\hline E2 & 82.407 & $81-84$ & $18-21$ & 40 \\
\hline $\mathrm{F} 2$ & 87.307 & $85-89$ & $22-26$ & 41 \\
\hline F\#2 & 92.499 & $90-95$ & $27-32$ & 42 \\
\hline G2 & 97.999 & $96-100$ & $33-37$ & 43 \\
\hline $\mathrm{G} \# 2$ & 103.826 & $101-106$ & $38-43$ & 44 \\
\hline $\mathrm{A} 2$ & 110 & 107-113 & $44-50$ & 45 \\
\hline $\mathrm{A} \# 2$ & 116.541 & $114-120$ & $51-57$ & 46 \\
\hline B2 & 123.471 & $121-127$ & $58-64$ & 47 \\
\hline C3 & 130.813 & $128-134$ & $65-71$ & 48 \\
\hline $\mathrm{CA3}$ & 138.591 & $135-142$ & $72-79$ & 49 \\
\hline D3 & 146.832 & $143-151$ & $80-88$ & 50 \\
\hline $\mathrm{D \# 3}$ & 155.564 & $152-160$ & $89-97$ & 51 \\
\hline E3 & 164.814 & $161-169$ & $98-106$ & 52 \\
\hline F3 & 174.614 & $170-179$ & $107-116$ & 53 \\
\hline $\mathrm{F} \# 3$ & 184.997 & $180-190$ & $117-127$ & 54 \\
\hline G3 & 195.998 & $191-201$ & $128-138$ & 55 \\
\hline $\mathrm{G} \# 3$ & 207.652 & $202-213$ & $139-150$ & 56 \\
\hline A3 & 220 & $214-226$ & 151-163 & 57 \\
\hline $\mathrm{A} \# 3$ & 233.082 & $227-240$ & $164-177$ & 58 \\
\hline B3 & 246.942 & $241-254$ & $178-191$ & 59 \\
\hline $\mathrm{C} 4$ & 261.626 & $255-269$ & $192-206$ & 60 \\
\hline $\mathrm{C} \# 4$ & 277.183 & $270-285$ & $207-222$ & 61 \\
\hline D4 & 293.665 & $286-302$ & $223-239$ & 62 \\
\hline $\mathrm{D} \# 4$ & 311.127 & $303-320$ & $240-257$ & 63 \\
\hline E4 & 329.628 & $321-339$ & $258-276$ & 64 \\
\hline F4 & 349.228 & $340-359$ & $277-296$ & 65 \\
\hline $\mathrm{F} \# 4$ & 369.994 & $360-380$ & $297-317$ & 66 \\
\hline G4 & 391.995 & $381-403$ & $318-340$ & 67 \\
\hline $\mathrm{G} \# 4$ & 415.305 & $404-427$ & $341-364$ & 68 \\
\hline $\mathrm{A} 4$ & 440 & $438-453$ & $365-390$ & 69 \\
\hline $\mathrm{A} \# 4$ & 466.164 & $454-480$ & $391-417$ & 70 \\
\hline B4 & 493.883 & $481-508$ & $418-445$ & 71 \\
\hline $\mathrm{C5}$ & 523.251 & $509-538$ & $446-475$ & 72 \\
\hline $\mathrm{C} \# 4$ & 554.365 & $539-570$ & $476-507$ & 73 \\
\hline D5 & 587.33 & $571-604$ & $508-541$ & 74 \\
\hline $\mathrm{D \# 4}$ & 622.254 & $605-640$ & $542-577$ & 75 \\
\hline E5 & 659.255 & $641-678$ & $578-615$ & 76 \\
\hline F5 & 698.457 & $679-719$ & $616-656$ & 77 \\
\hline $\mathrm{F} \# 5$ & 739.989 & $720-761$ & $657-698$ & 78 \\
\hline G5 & 783.991 & $762-807$ & $699-744$ & 79 \\
\hline $\mathrm{G} \# 4$ & 830.609 & 808-855 & $745-792$ & 80 \\
\hline A5 & 880 & $856-906$ & $793-843$ & 81 \\
\hline $\mathrm{A} \# 5$ & 932.328 & $907-960$ & $844-897$ & 82 \\
\hline B5 & 987.767 & 961-1017 & $898-954$ & 83 \\
\hline C6 & 1046.502 & $1018-1078$ & $955-1015$ & 84 \\
\hline
\end{tabular}

Table 4.2: Pitches corresponding to actual fundamental frequencies, valid integer representations from the AMDF, linked list memory offsets, and MIDI note numbers. All numbers are in base 10. Red shaded areas represent pitches outside the normal human vocal range. The blue shaded area is middle $C$ on the piano. 
This conversion method is inefficient regarding memory use because the linked list required $1016_{\mathrm{dec}}$ entries, but there is plenty of memory available. However, the method is extremely efficient regarding speed because the computational complexity for finding the appropriate MIDI note number is $\mathrm{O}(1)$. This works because there is only one MIDI note number for each frequency, whereas there may be multiple frequencies for each MIDI note number.

\subsubsection{Sending a Note to the Synthesizer (SENDMIDI)}

This routine is very unique in that it is the only routine that is continually executed outside the interrupt service routine (ADCIRQ). The reason for this is simply because the ACIA itself forms a bottleneck in this design. One may recall from Section 2.4.4 that the minimum time required to send a MIDI message of three bytes length is $0.96 \mathrm{~ms}$. This poses a problem because the sampling routine triggers every $1.000 \mathrm{~ms}$. Therefore, this routine must be placed outside the sampling routine and called continuously in a never-ending while-loop. A simple flag set in the FAVG routine (Section 4.2.5) will be used to determine whether a note is to be sent to the synthesizer, the current note is to be turned off, and if a new note is to be sent to the synthesizer. Figure 3.9 illustrates the functionality of one routine via a flow diagram. 


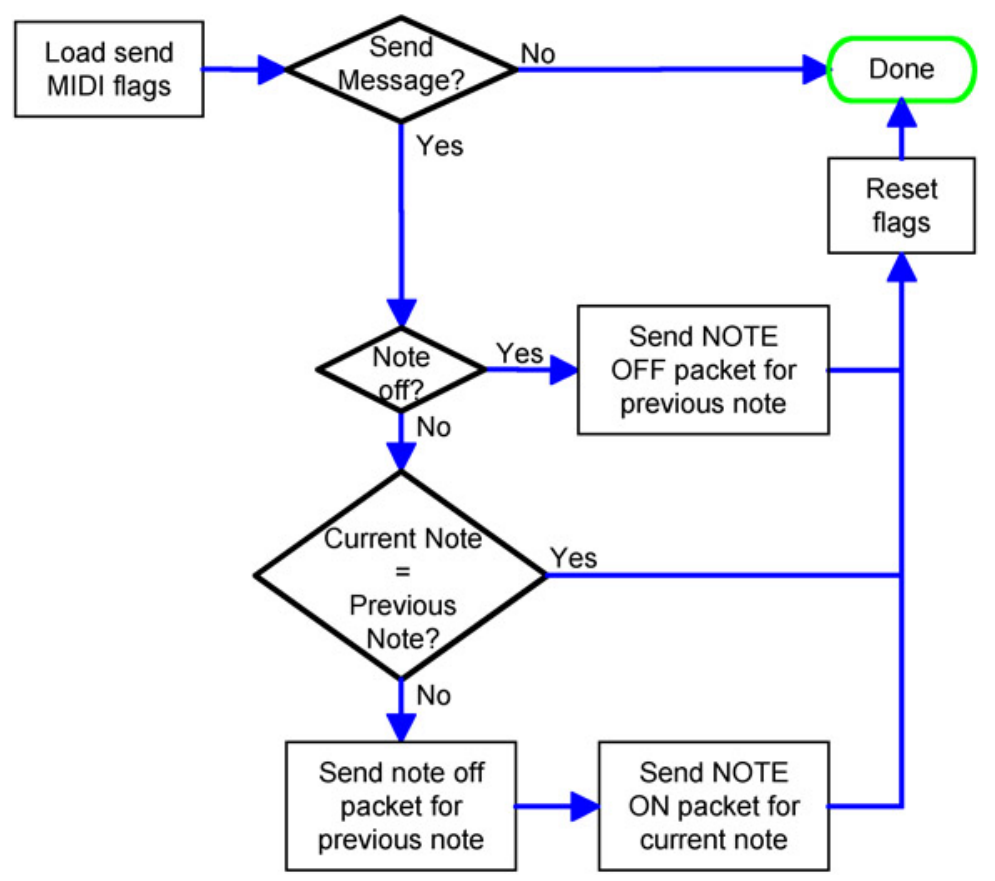

Figure 4.7: Flow diagram for sending MIDI commands.

Aside from the timing considerations, this routine analyzes a variable called MIDIFLAG to determine what packets are to be sent. The first check determines if any packets are to be sent at all. If not, then the routine exits. The second check determines if the previous note is to be disabled without sending a new note to the synthesizer. This is useful if the user quits singing. The next check determines if the previous note needs to be disabled with the intentions of sending a new note (on command) to the synthesizer.

If a note is to be enabled or disabled, the routine takes the note number specified in the MIDINOTE variable and formats a MIDI protocol packet. This packet is sent to the synthesizer using the MIDI ACIA. Recall that the packet format for turning a note on or off is as follows: 


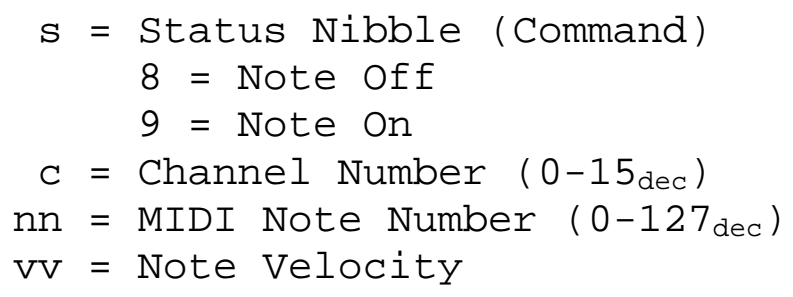

Each time this routine is called with the intentions of sending a new note, the note off MIDI command is sent with the previously activated note (PREVNOTE) if the previous note differs from the current note. Then the value of the current note, stored in MIDINOTE, is loaded into a data register. The current note is sent with the note on MIDI command, and the current value of MIDINOTE is saved in the variable PREVNOTE, so that it may be turned off during the next instance of SENDMIDI. Before the routine exits, the flags are reset so no additional data is accidentally transmitted when unnecessary. 


\section{CHAPTER V - RESULTS}

\subsection{MATLAB AMDF Proof of Concept Simulations}

The AMDF algorithm was simulated using MATLAB v7.0 and pre-recorded Windows audio (wav) files set to record 8-bit samples on a single channel at the minimum frequency the software allowed $(8.000 \mathrm{kHz})$. This data was down-sampled to 4.0, 2.0, 1.6 and $1.0 \mathrm{kHz}$ to by removing every second, fourth, fifth and eight sample, respectively, to better approximate the sampling rate to be used for the final design. To simulate the hardware design, the sample data was then half-wave rectified by setting all data with negative amplitude equal to zero. Although the half-wave rectification is nonlinear and introduces additional harmonics, the AMDF performed similarly to the nonrectified case in the preliminary simulations. MATLAB returns wav file values in the range of minus one to positive one and so the data were scaled by 255 to simulate the output from the A/D converter. Finally, all floating-point numbers were rounded down to simulate unsigned bytes from the $\mathrm{A} / \mathrm{D}$ converter.

The AMDF code obviously outputs the reported fundamental frequency for a selected data segment of length $\mathrm{K}_{\mathrm{MAX}}+\mathrm{N}_{\operatorname{MAX}}$. However, it is necessary to compare this output to another frequency measure of the same data. This is accomplished by computing the power spectrum of the same data segment by using a 1024 point FFT. Once computed, maxima that appear above a certain threshold were calculated and their frequencies recorded. These recorded frequencies were compared to the output of the AMDF. This process is repeated for 12 data segments for each file. Each new segment uses the last $\mathrm{K}_{\mathrm{MAX}}$ entries of the previous data segment to better represent how the assembly code will function. Additionally, the same 12 data segments for each file were 
analyzed for sampling frequencies of 4.0, 2.0, 1.6 and $1.0 \mathrm{kHz}$. Finally, three files were analyzed with a male signer singing tones approximating F3 $(174.614 \mathrm{~Hz})$, A3 $(220 \mathrm{~Hz})$ and C4 (261.626). This set of simulations is by no means an exhaustive set of tests, but gives an idea of the success or failure of the algorithm. The code responsible for this can be seen in Appendix II and the results can be seen in Figures 5.1-5.4 and Tables 5.1-5.3.

The initial simulation results (not shown) were very disappointing. However, varying $\mathrm{K}_{\mathrm{MAX}}$ and $\mathrm{N}_{\mathrm{MAX}}$ varied performance of the AMDF. In the results presented in Tables 5.1-5.3, the values of $\mathrm{K}_{\mathrm{MAX}}$ and $\mathrm{N}_{\mathrm{MAX}}$ had to be reduced for the lower sampling frequencies, else the reported results had an extremely high error. Ultimately, using an adaptive window and adaptive cutoff threshold would increase the AMDF performance [Kim 1998]. Figures 5.1-5.4 graphically show simulation results for one iteration of the "A3.wav" file sampled at each of the four sampling frequencies. In each figure, there are three subfigures. The top subfigures show the half-wave rectified input signal, where the sample number is represented on the horizontal axis and signal amplitude is represented on the vertical axis. The middle subfigures show the results of the AMDF as applied to the data in the top subfigures. In the middle subfigures, the horizontal axis represents the sample number, the vertical axis represents the AMDF results, and the green line represents the threshold for identifying minima relevant to the frequency identification. The bottom subfigures show the power spectra for the data from the top subfigures, where the horizontal axis represents the frequency in $\mathrm{Hz}$, and the vertical axis is the magnitude of the power spectra. 
/2 Wave Rectification

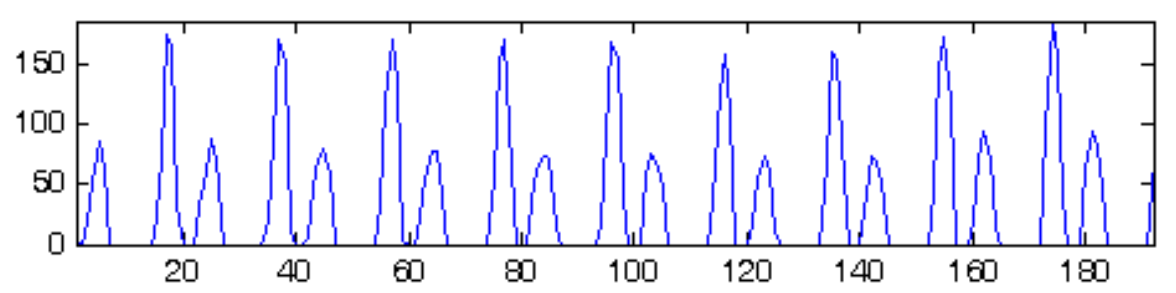

Sampling a. wav at $4000 \mathrm{~Hz}$, results: $T=6.54167$ samples, $F=611.465 \mathrm{~Hz}$

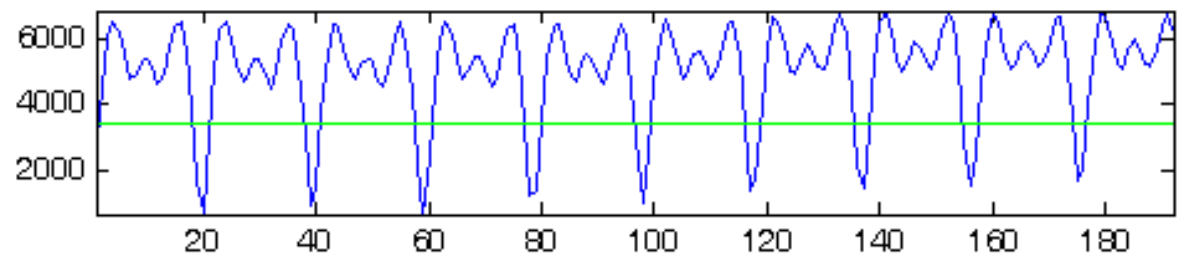

$\times 10^{4}$ Power spectra for a. wav with spikes at $199.22,207.03,410.16,617.19 \mathrm{~Hz}$

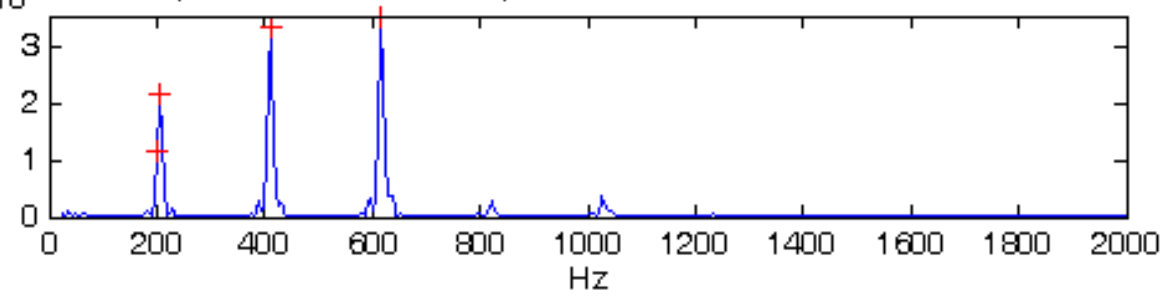

Figure 5.1: $A M D F$ simulation results for $A 3, f_{s}=4.0 \mathrm{kHz}$.

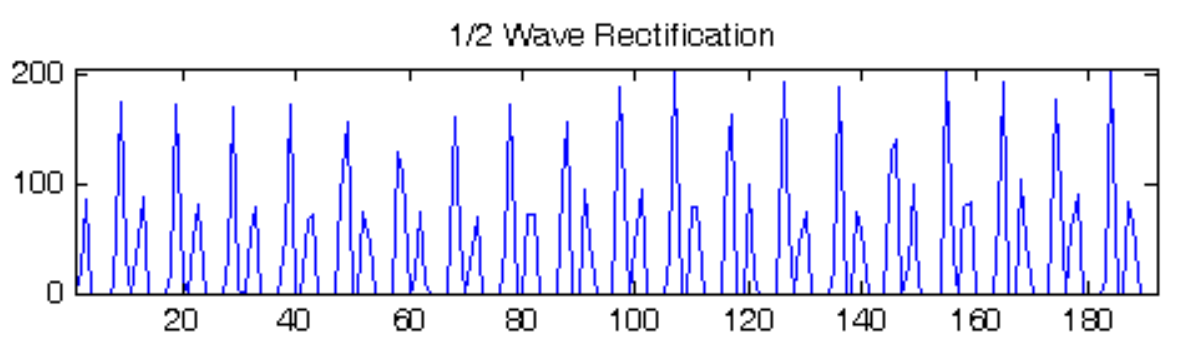

Sampling a wav at $2000 \mathrm{~Hz}$, results: $T=8.4$ samples, $F=238.095 \mathrm{~Hz}$

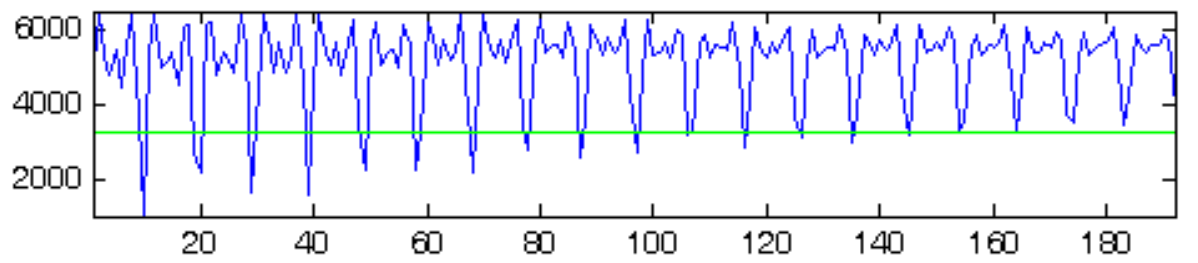

$\times 10^{4} \quad$ Power spectra for a. wav with spikes at $207.03,416.02,623.05 \mathrm{~Hz}$

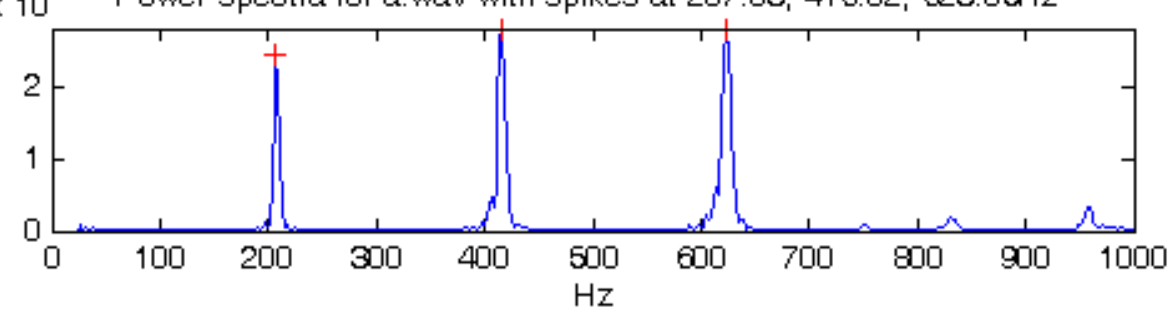

Figure 5.2: $A M D F$ simulation results for $A 3, f_{s}=2.0 \mathrm{kHz}$. 
1/2 Wave Rectification

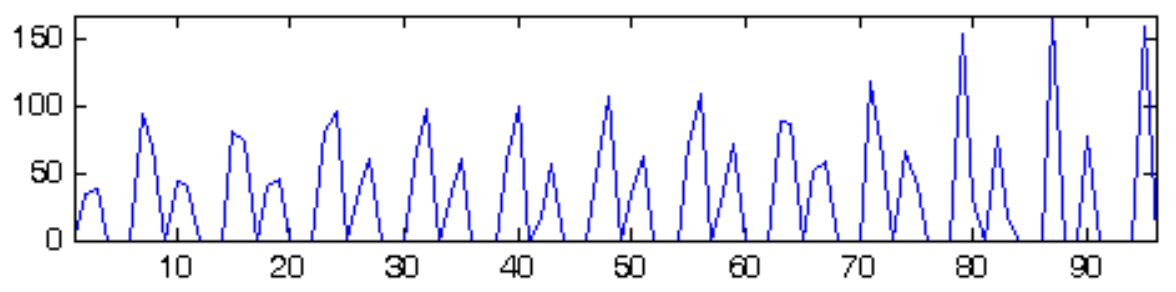

Sampling a. wav at $1600 \mathrm{~Hz}$, results: $\mathrm{T}=7.875$ samples, $F=203.175 \mathrm{~Hz}$

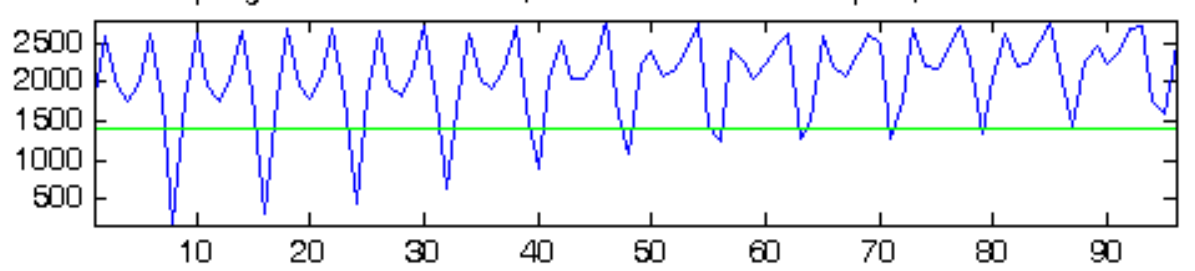

Power speotra for a. wav with spikes at 198.44, 203.13,406.25, 610.94 Hz

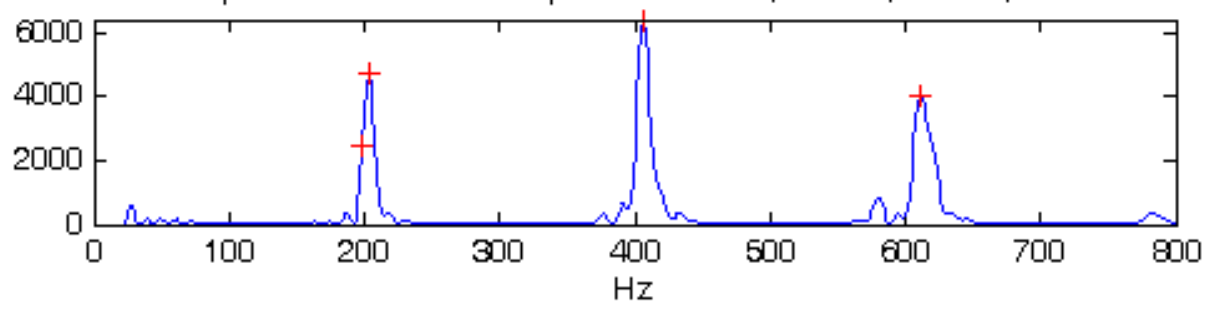

Figure 5.3: AMDF simulation results for $A 3, f_{s}=1.60 \mathrm{kHz}$.

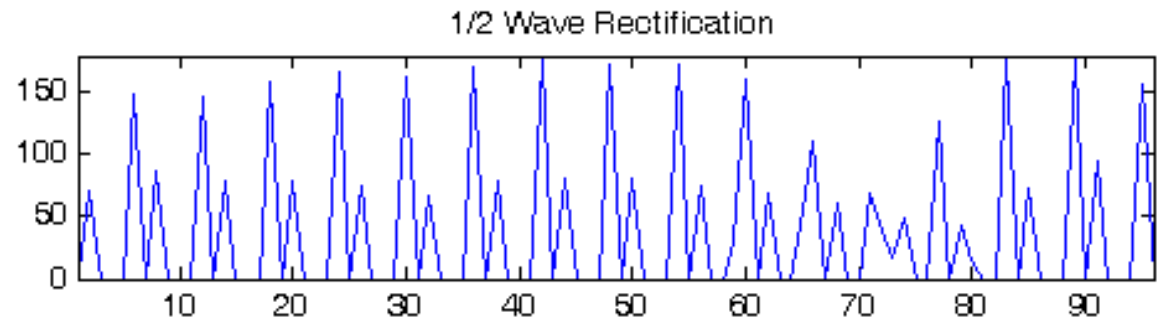

Sampling a. wav at $1200 \mathrm{~Hz}$, results: $T=6.90909$ samples, $F=173.684 \mathrm{~Hz}$
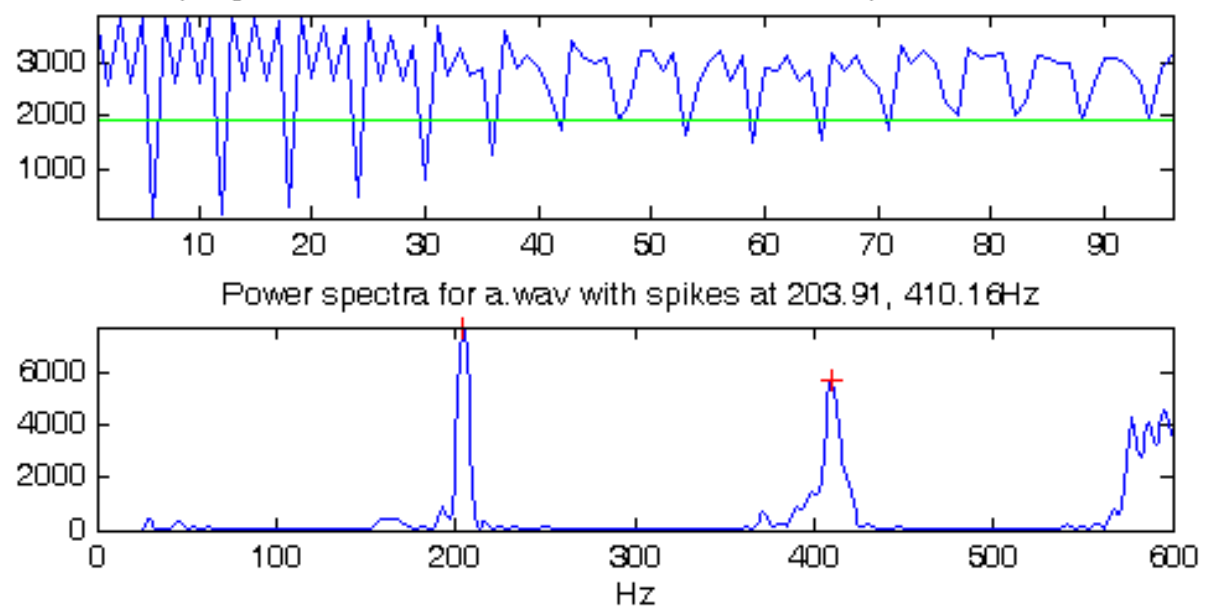

Figure 5.4: AMDF simulation results for A3, $f_{s}=1.0 \mathrm{kHz}$. 


\begin{tabular}{|c|c|c|c|c|c|}
\hline & & \multicolumn{4}{|c|}{ 'f.wav' - Sampling Frequency (Hz) } \\
\hline & Iteration & 4000 & 2000 & 1600 & 1000 \\
\hline \multirow{12}{*}{$\sum_{<}^{\frac{1}{2}}$} & 1 & 606.061 & 193.182 & 213.333 & 166.667 \\
\hline & 2 & 602.41 & 237.288 & 256 & 166.667 \\
\hline & 3 & 598.802 & 269.663 & 210.526 & 148.936 \\
\hline & 4 & 550.898 & 239.521 & 231.579 & 150.943 \\
\hline & 5 & 550.898 & 309.524 & 231.579 & 144.578 \\
\hline & 6 & 571.429 & 285.714 & 190.476 & 144.578 \\
\hline & 7 & 571.429 & 272.189 & 213.333 & 144.578 \\
\hline & 8 & 571.428 & 331.361 & 207.059 & 144.578 \\
\hline & 9 & 520.71 & 261.905 & 188.235 & 132.53 \\
\hline & 10 & 544.379 & 214.286 & 169.412 & 120.482 \\
\hline & 11 & 520.71 & 273.81 & 169.412 & 166.667 \\
\hline & 12 & 497.041 & 292.994 & 169.412 & 83.333 \\
\hline \multirow{12}{*}{$\stackrel{\leftarrow}{\square}$} & 1 & 171.88 & 169.92 & 171.88 & 169.92 \\
\hline & 2 & 171.88 & 169.92 & 170.31 & 169.92 \\
\hline & 3 & 167.97 & 167.97 & 170.31 & 168.95 \\
\hline & 4 & 167.97 & 167.97 & 170.31 & 168.95 \\
\hline & 5 & 167.97 & 167.97 & 168.75 & 168.95 \\
\hline & 6 & 167.97 & 167.97 & 168.75 & 167.97 \\
\hline & 7 & 167.97 & 167.97 & 168.75 & 167.97 \\
\hline & 8 & 167.97 & 166.02 & 168.75 & 167.97 \\
\hline & 9 & 167.97 & 166.02 & 168.75 & 167.97 \\
\hline & 10 & 167.97 & 166.02 & 168.75 & 167.97 \\
\hline & 11 & 167.97 & 166.02 & 168.75 & 166.99 \\
\hline & 12 & 164.06 & 166.02 & 168.75 & 166.99 \\
\hline \multirow{12}{*}{ 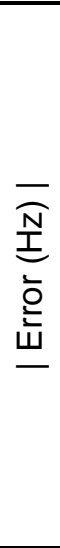 } & 1 & 434.181 & 23.262 & 41.453 & 3.253 \\
\hline & 2 & 430.53 & 67.368 & 85.69 & 3.253 \\
\hline & 3 & 430.832 & 101.693 & 40.216 & 20.014 \\
\hline & 4 & 382.928 & 71.551 & 61.269 & 18.007 \\
\hline & 5 & 382.928 & 141.554 & 62.829 & 24.372 \\
\hline & 6 & 403.459 & 117.744 & 21.726 & 23.392 \\
\hline & 7 & 403.459 & 104.219 & 44.583 & 23.392 \\
\hline & 8 & 403.458 & 165.341 & 38.309 & 23.392 \\
\hline & 9 & 352.74 & 95.885 & 19.485 & 35.44 \\
\hline & 10 & 376.409 & 48.266 & 0.662 & 47.488 \\
\hline & 11 & 352.74 & 107.79 & 0.662 & 0.323 \\
\hline & 12 & 332.981 & 126.974 & 0.662 & 83.657 \\
\hline
\end{tabular}

* NMAX $=192, \mathrm{KMAX}=96$ for fs $=4000$ and $2000 \mathrm{~Hz}$.

** NMAX $=96, \mathrm{KMAX}=48$ for fs $=1600$ and $1000 \mathrm{~Hz}$.

Table 5.1: AMDF simulation results from MATLAB for F3. 


\begin{tabular}{|c|c|c|c|c|c|}
\hline & & \multicolumn{4}{|c|}{ 'a.wav' - Sampling Frequency $(\mathrm{Hz})$} \\
\hline & Iteration & 4000 & 2000 & 1600 & 1000 \\
\hline \multirow{12}{*}{$\sum_{<}^{U}$} & 1 & 493.827 & 200 & 200 & 189.873 \\
\hline & 2 & 621.118 & 237.288 & 200 & 188.406 \\
\hline & 3 & 600 & 285.714 & 222.785 & 180.723 \\
\hline & 4 & 607.595 & 241.379 & 202.532 & 186.441 \\
\hline & 5 & 611.465 & 238.095 & 203.175 & 166.667 \\
\hline & 6 & 589.744 & 277.457 & 205.128 & 158.73 \\
\hline & 7 & 554.913 & 263.736 & 205.128 & 181.818 \\
\hline & 8 & 551.724 & 243.94 & 225.641 & 137.931 \\
\hline & 9 & 574.713 & 287.293 & 205.128 & 137.931 \\
\hline & 10 & 528.736 & 287.293 & 228.571 & 168.831 \\
\hline & 11 & 574.713 & 266.667 & 225.571 & 151.163 \\
\hline & 12 & 574.713 & 266.667 & 207.792 & 139.535 \\
\hline \multirow{12}{*}{$\stackrel{\leftarrow}{\square}$} & 1 & 199.22 & 203.13 & 198.44 & 203.13 \\
\hline & 2 & 199.22 & 205.08 & 198.44 & 199.22 \\
\hline & 3 & 199.22 & 205.08 & 198.44 & 204.1 \\
\hline & 4 & 199.22 & 207.03 & 198.44 & 204.1 \\
\hline & 5 & 199.22 & 207.03 & 198.44 & 205.08 \\
\hline & 6 & 199.22 & 207.03 & 198.44 & 205.08 \\
\hline & 7 & 207.03 & 208.98 & 198.44 & 206.05 \\
\hline & 8 & 207.03 & 208.98 & 204.69 & 206.05 \\
\hline & 9 & 207.03 & 208.98 & 204.69 & 206.05 \\
\hline & 10 & 207.03 & 210.94 & 206.25 & 207.03 \\
\hline & 11 & 207.03 & 210.94 & 206.25 & 208.01 \\
\hline & 12 & 210.94 & 210.94 & 206.25 & 208.01 \\
\hline \multirow{12}{*}{ 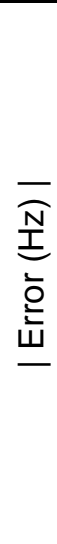 } & 1 & 294.607 & 3.13 & 1.56 & 13.257 \\
\hline & 2 & 421.898 & 32.208 & 1.56 & 10.814 \\
\hline & 3 & 400.78 & 80.634 & 24.345 & 23.377 \\
\hline & 4 & 408.375 & 34.349 & 4.092 & 17.659 \\
\hline & 5 & 412.245 & 31.065 & 4.735 & 38.413 \\
\hline & 6 & 390.524 & 70.427 & 6.688 & 46.35 \\
\hline & 7 & 347.883 & 54.756 & 6.688 & 24.232 \\
\hline & 8 & 344.694 & 34.96 & 20.951 & 68.119 \\
\hline & 9 & 367.683 & 78.313 & 0.438 & 68.119 \\
\hline & 10 & 321.706 & 76.353 & 22.321 & 38.199 \\
\hline & 11 & 367.683 & 55.727 & 19.321 & 56.847 \\
\hline & 12 & 363.773 & 55.727 & 1.542 & 68.475 \\
\hline
\end{tabular}

* NMAX $=192, \mathrm{KMAX}=96$ for fs $=4000$ and $2000 \mathrm{~Hz}$.

** NMAX $=96, \mathrm{KMAX}=48$ for fs $=1600$ and $1000 \mathrm{~Hz}$.

Table 5.2: AMDF simulation results from MATLAB for A3. 


\begin{tabular}{|c|c|c|c|c|c|}
\hline & & \multicolumn{4}{|c|}{ 'c.wav' - Sampling Frequency (Hz) } \\
\hline & Iteration & 4000 & 2000 & 1600 & 1000 \\
\hline \multirow{12}{*}{$\sum_{<}^{L}$} & 1 & 906.077 & 254.237 & 290.909 & 250 \\
\hline & 2 & 491.228 & 285.714 & 256.79 & 142.857 \\
\hline & 3 & 564.706 & 285.714 & 217.284 & 131.579 \\
\hline & 4 & 840.909 & 268.657 & 237.037 & 117.647 \\
\hline & 5 & 666.667 & 292.683 & 217.284 & 160 \\
\hline & 6 & 613.333 & 352.941 & 216.216 & 172.414 \\
\hline & 7 & 640 & 57.143 & 220 & 108.108 \\
\hline & 8 & 601.77 & 205.714 & 240 & 250 \\
\hline & 9 & 571.429 & 262.857 & 241.509 & 250 \\
\hline & 10 & 666.667 & 148.571 & 241.509 & 81.633 \\
\hline & 11 & 617.143 & 170.455 & 272.34 & 122.449 \\
\hline & 12 & 545.455 & 181.818 & 240 & 58.824 \\
\hline \multirow{12}{*}{$\stackrel{\leftarrow}{\square}$} & 1 & 468.75 & 472.66 & 470.31 & 241.21 \\
\hline & 2 & 468.75 & 251.95 & 470.31 & 241.21 \\
\hline & 3 & 472.66 & 253.91 & 470.31 & 241.21 \\
\hline & 4 & 242.19 & 248.05 & 471.88 & 241.21 \\
\hline & 5 & 242.19 & 250 & 473.44 & 290.04 \\
\hline & 6 & 246.09 & 250 & 240.63 & 239.26 \\
\hline & 7 & 250 & 251.95 & 240.63 & 254.88 \\
\hline & 8 & 250 & 251.95 & 242.19 & 254.88 \\
\hline & 9 & 253.91 & 251.95 & 243.75 & 253.91 \\
\hline & 10 & 253.91 & 250 & 245.31 & 253.91 \\
\hline & 11 & 253.91 & 250 & 246.88 & 253.91 \\
\hline & 12 & 250 & 250 & 248.44 & 252.93 \\
\hline \multirow{12}{*}{ 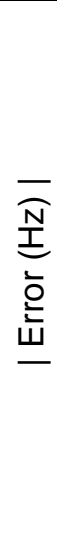 } & 1 & 437.327 & 218.423 & 179.401 & 8.79 \\
\hline & 2 & 22.478 & 33.764 & 213.52 & 98.353 \\
\hline & 3 & 92.046 & 31.804 & 253.026 & 109.631 \\
\hline & 4 & 598.719 & 20.607 & 234.843 & 123.563 \\
\hline & 5 & 424.477 & 42.683 & 256.156 & 130.04 \\
\hline & 6 & 367.243 & 102.941 & 24.414 & 66.846 \\
\hline & 7 & 390 & 194.807 & 20.63 & 146.772 \\
\hline & 8 & 351.77 & 46.236 & 2.19 & 4.88 \\
\hline & 9 & 317.519 & 10.907 & 2.241 & 3.91 \\
\hline & 10 & 412.757 & 101.429 & 3.801 & 172.277 \\
\hline & 11 & 363.233 & 79.545 & 25.46 & 131.461 \\
\hline & 12 & 295.455 & 68.182 & 8.44 & 194.106 \\
\hline
\end{tabular}

* NMAX $=192, \mathrm{KMAX}=96$ for fs $=4000$ and $2000 \mathrm{~Hz}$.

** NMAX $=96, \mathrm{KMAX}=48$ for fs $=1600$ and $1000 \mathrm{~Hz}$.

Table 5.3: AMDF simulation results from MATLAB for C4. FFT errors are shown in italicized red. 
These results indicate that the real-time implementation of this device will have errors that will likely be perceptible to the user. The better performances of the AMDF are at best $+/$ - a musical half step from the intended frequency, while other errors are up to two octaves off. Ideally, a faster processor would yield better results because additional techniques could be implemented to correct the errors.

\subsection{Easy68K Simulations}

The next logical step is to simulate the assembly level implementation before using the constructed hardware. There are two ultimate goals for these simulations. The first goal is to ensure that the assembly code results match the MATLAB simulation results for the same data set, aside from rounding. The second goal is to ensure that the code execution time is short enough to operate between sampling periods.

The assembly implementation must be modified slightly to accommodate the fact that the data are not sampled in real time with a microphone. This was accomplished by saving the source data used in the MATLAB simulations to an assembly file (s.x68). The data was added to the bottom of the program space in the code. Then the interrupt routine was modified so that the data was copied from each subsequent entry in the $\mathrm{s}()$ array instead of from the ADC location.

Once these modifications were complete, breakpoints were set in the code and were executed to view intermediate results. Ultimately, this helped in the code debugging process, as there were a few logical problems that needed to be resolved. Once the coding errors were corrected, the results could be observed. Initial results proved promising, in that the observed frequency matched the frequency identified in 
MATLAB in all cases, excepting the rounding error in the final step. All the cases described in Section 5.1 were tested and the results matched in each case.

The Easy $68 \mathrm{~K}$ also has a tool that measures the number of clock cycles between instructions. This tool is used to determine if the timing is correct between interrupt requests. Ultimately, the goal is to ensure that the number of clock cycles is less than 8000 if a $1.0 \mathrm{kHz}$ sampling frequency is used or 5000 if a $1.6 \mathrm{kHz}$ sampling frequency is used. Initially, these tests proved that the code would not run in the time allotted because the average number of clock cycles between samplings was around $9000+/-10 \%$ clock cycles. Upon further investigation, the code appeared to be inefficient, so it was rewritten with more efficient uses of commands than before. This accounted for a reduction of clock cycles to $6000+/-10 \%$ with the same settings for $\mathrm{K}_{\mathrm{MAX}}$ and $\mathrm{N}_{\mathrm{MAX}}$, a $50 \%$ increase in efficiency.

Ultimately, this simulation tool saved countless hours of debugging in the lab. Code revisions could be implemented and simulated quickly, without the need for using the emulator or hardware. This simulator also saved time in the sense that the code was simulated while the hardware prototype was still being constructed.

\subsection{Hardware Debugging with the Deneb Emulator}

After constructing the physical hardware as described in Section 4.1, it was necessary to test the hardware in two stages to ensure the prototype matched the schematics and board layout before moving on to the final design implementation. The first stage involved checking all the connections formed by tracks and wires. The second stage involved checking the various modules with the memory dump feature of the Deneb Emulator software and with software segments written in assembly. 


\subsubsection{Checking Physical Connections}

In general, soldered connections can have a few types of failures. The first is lack of continuity (open circuit), the second is a short between neighboring pads/wires/pins, and the third is internal quasi-open circuits formed by capacitance, which can be formed by "cold" solder joints". Although checks of suspicious connection were made during construction, there are inevitably additional errors for a large design.

The first and most tedious task is checking all the connections between devices to ensure all part are connected according to the schematic. Performing a continuity check with a digital multi-meter (DMM) with all the parts removed from their respective sockets checks for open circuits well. Checking for short circuits is a little more difficult because each pin of one device must be checked against every other pin on every other device to check each connection. Instead of checking every combination of pins, only neighboring pins were checked for shorts because most shorts come from wires and solder crossing pads. The final test involved measuring the capacitance between connections. The lines most susceptible to error because of too much capacitance are the data and address lines; hence these were the only lines check for cold solder joints outside of a purely visual inspection of all connections.

\subsubsection{Memory}

Memory is perhaps the easiest component to test outside of the manual tests described above because all one has to do is connect the emulator, then read and write to the memory dump window. Once the power was applied to the board and the emulator

\footnotetext{
5 "cold" solder joints are formed when insufficient heat is applied to the physical components soldered.
} 
configured, the memory did not work as expected. The dump window read the values corresponding to floating values on the data lines for both the even and odd address space. This meant that either the memory was bad or the MMU was not operating properly, the latter being the most likely cause. The memory select lines (SERAM' and SORAM') were not asserting when the corresponding addresses were accessed in the dump window. Further investigation revealed that address strobe (AS') was connected to $\mathrm{V}_{\mathrm{CC}}(5 \mathrm{~V})$, thereby constantly disabling both decoders. Once the connection was restored, $\mathrm{read} / \mathrm{write}$ functionality was possible.

This design relies on the non-volatility of the RAM, as there is no other way to program the prototype without adding additional hardware or purchasing additional components. The only tests of interest are to (1) see if the RAM will hold values with the noise present on the board and to (2) see if the NVRAM holds its values once power is cycled. Both tests had positive results.

\subsubsection{Bar graph LEDs}

Upon powering the board, the LEDs light up if values are present on the data lines. Testing the LEDs is a simple as testing the memory, with the exception that the data written to the register driving the LEDs will not appear in the dump window because the hardware design would be needlessly more complicated. The other difference is that all bits written must be inverted to light the appropriate LEDs. When this test was performed, one observed inconvenience was that after the data was written, the values on the LEDs changed from the input value, mainly because the emulator attempts to read many values to update the window. 
It was necessary to write a small segment of code to test the LEDs. This code is attached in Appendix III. The test simply lit a single LED, and performed a one-bit logical shift after a short delay, and repeated indefinitely. This test revealed that the first and third LEDs were shorted, a test not revealed by checking neighbors for shorts. The short was fixed and the LEDs functioned properly thereafter.

\section{$\underline{5.3 .4 \mathrm{~A} / \mathrm{D} \text { converter }}$}

The $\mathrm{A} / \mathrm{D}$ converter testing required the addition of extra hardware as seen in Figure 5.1. The hardware consisted of a simple 10k potentiometer with the end terminals connected to $\mathrm{V}_{\mathrm{CC}}(5 \mathrm{~V})$ and ground. The wiper terminal was connected to IN0 on the ADC0809. By varying the position of the wiper, the voltage between the wiper terminal and ground should vary linearly between 0 and $5 \mathrm{~V}$. Similarly, the reading from the A/D converter readings should respectively vary between 0 and $255_{\mathrm{dec}}$.

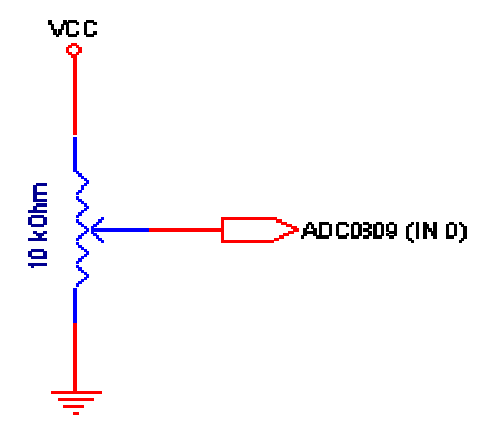

Figure 5.5: A/D converter test circuit.

This module is similar to the LEDs in that the value read from the memory dump window will not be the same as the one written. To initiate the A/D conversion, any value may be written to the device. When the emulator immediately reads the device after the write operation, the previous conversion results are displayed in the window. To perform the test, the wiper on the potentiometer is varied a number of times, the resulting 
voltages are measured with a DMM, and the equivalent decimal values are calculated for comparison. The A/D converter functions as expected with the potentiometer. After this test was completed, the additional hardware was removed.

\section{$\underline{\text { 5.3.5 Power-on reset circuitry, serial communications interface (SCI), }}$ and sampling frequency generator interrupt circuitry}

By this point, the timing associated with the power-on reset circuit has been tested and the results are discussed in more detail in Section 3.1.12 and shown in Figure 3.16. However, it is necessary to test whether the reset switch and the power-on reset functionality cause the 68000 to reset as predicted. Any simple program should begin immediate execution once power is applied or when the reset pushbutton is depressed, so this test is coupled with some others.

An easy way to verify the interrupt and SCI circuitry is working properly is to send a small message via the SCI. This message must be small so as not to cause a backlog of interrupt service requests. It takes 10 bits to send a single byte to the terminal. At 38400 bps, a total of 3.84 bytes may be sent between interrupt requests if the interrupts occur every $1 \mathrm{~ms}$. Once the 68000 comes out of reset, a message to clear the terminal screen is sent via the SCI. Then, the interrupts are enabled and a test message with the string "TST" should be sent to the terminal with each interrupt request. As expected, when power is supplied, the terminal screen is cleared and begins to fill with "TST". The same is true if the reset pushbutton is depressed at any time.

\subsubsection{MIDI interface}

The last major module to check is the MIDI interface. The easiest way to do this is to send properly formatted packets through the MIDI SCI. The messages selected are a 
program change to set the instrument to Grand Piano and a series of messages to play the C-major scale followed by a C-major I chord then a command to stop the notes. This routine is set to execute once and the code is attached in Appendix III.

Overall, this interface was the most difficult to debug in hardware because the MIDI OUT line is not connected to the MIDI IN line of the other device, but rather the lines are connected to their respective names on the opposite devices. This is different from connecting the SCI communicating with a PC terminal. Nonetheless, the MIDI interface functionality was successfully verified.

\subsubsection{Proven code}

The last test, outside of testing the code that was developed and simulated, was to test the hardware with a large segment of proven assembly code. Loading and executing the Game of Snake performed this test. This particular game was written in 2004 as part of the Microcomputer Design course (ECE516) taught at the University of Louisville and its functionality has long since been proven to run on a similar hardware design. The code for this game was modified to run in a different addressing scheme and to update the LEDs to reflect the player's score in binary, which is also displayed on the terminal screen. After completing the modifications, the code ran perfectly on the emulator, which suggested that the prototype was ready to be tested in stand-alone mode, as discussed in the next section. 


\section{$\underline{\text { 5.3.8 Butterworth Filter }}$}

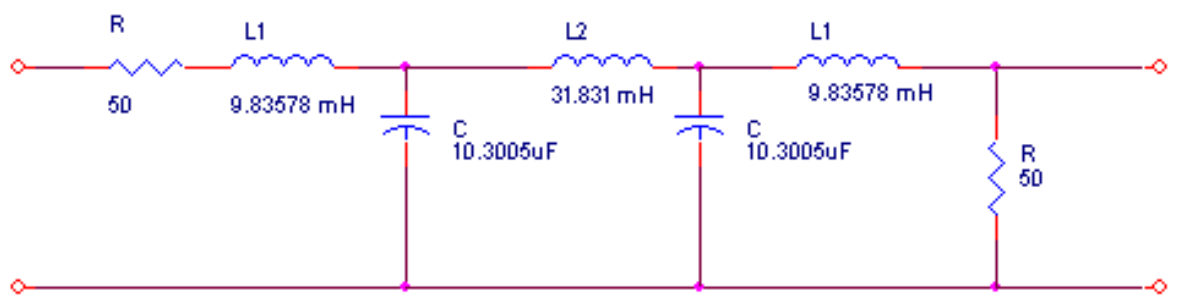

Figure 5.6: Fifth order Butterworth low pass filter design.

The fifth-order Butterworth low pass filter shown in Figure 5.6 is checked graphically measuring by the filter's amplitude response in a simulation and physically in the lab using a function generator with linear sweep and an oscilloscope with XY interpretation. The red curve in Figure 5.7 shows the results of a WinSpice simulation with the ideal values input from the website. This is not normalized, therefore the 3.01 $\mathrm{dB}$ point occurs at $\sqrt{2} / 4$ instead of the traditional $\sqrt{2} / 2$. From this waveform, it is easy to see that the filter reaches the appropriate amplitude at the desired cutoff frequency $(500 \mathrm{~Hz})$, and remains relatively flat until $350 \mathrm{~Hz}$. The source code to generate the plots can be found in Appendix II. 


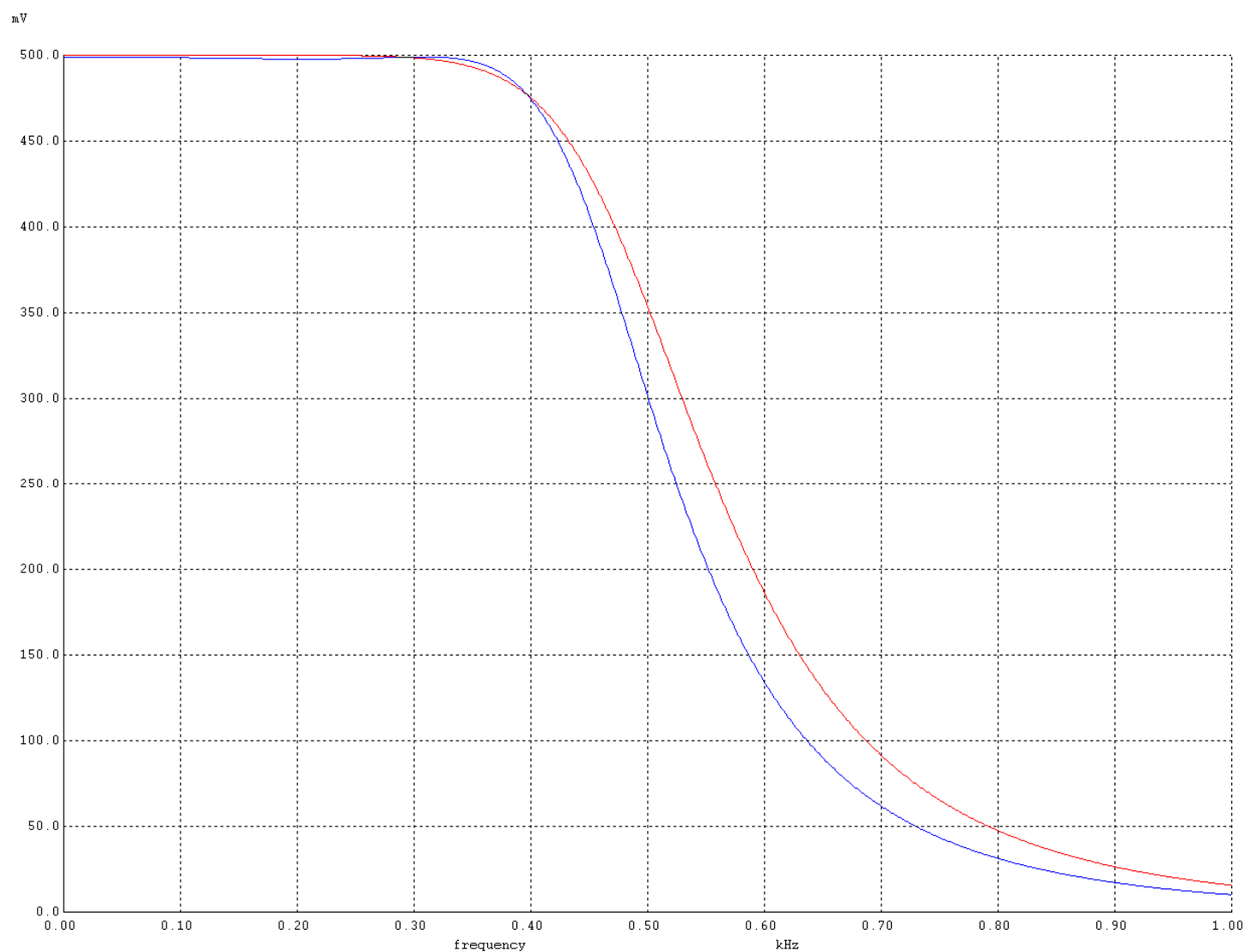

Figure 5.7: Butterworth filter frequency responses for ideal (red, gently sloped) and actual (blue, steeply sloped) component values.

The next step to test the Butterworth filter is to physically assemble the circuit and to measure the frequency response. The exact component values were not all available in the lab, so the closest approximations were used. The actual component values are $\mathrm{R}=$ $51.6 \Omega, \mathrm{L}_{1}=11.29 \mathrm{mH}, \mathrm{C}_{1}=11.45 \mu \mathrm{F}, \mathrm{L}_{2}=31.39 \mathrm{mH}, \mathrm{C}_{2}=11.45 \mu \mathrm{F}$, and $\mathrm{L}_{3}=11.35 \mathrm{mH}$. These values were simulated again using WinSpice and the anticipated results are shown in blue in Figure 5.7. From the results, it is anticipated that the cutoff frequency will be about $475 \mathrm{~Hz}$ instead of $500 \mathrm{~Hz}$.

The filter is then constructed on the prototyping board and is tested with a function generator capable of a linear frequency sweep. The sweep is varied from 1 to $1000 \mathrm{~Hz}$ and the results viewed in XY mode on the oscilloscope, which is shown in 
Figure 5.8. In this figure, the frequency drops to the $3.01 \mathrm{~dB}$ point at about $360 \mathrm{~Hz}$, which is not consistent with the simulations.

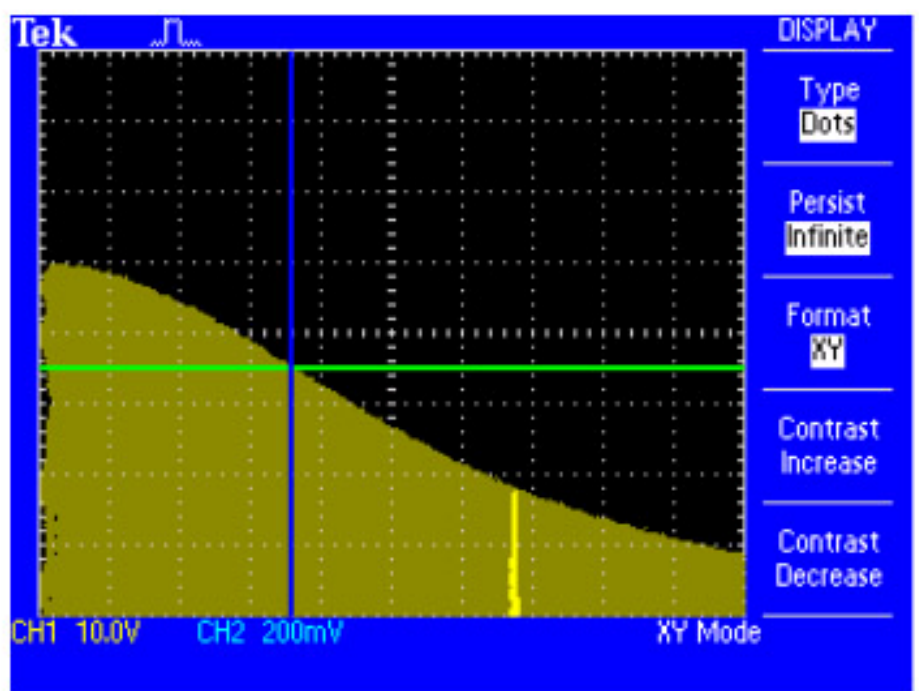

Figure 5.8: Actual Butterworth filter frequency response with $100 \mathrm{~Hz} /$ division horizontally and $200 \mathrm{mV} /$ division vertically. The intersecting green and blue lines represent the 3.01dB point.

The component values were measured again on the board itself and they matched the values reported above, except that the inductor values seem to vary by $+/-50 \%$ while connected to the bridge (Stanford Research Systems Model SR720 LCR Meter). A different bridge (GenRad 1657 RLC DIGIMETER) was used and the component values all matched except the $30 \mathrm{mH}$ inductor, which consistently read as $15 \mathrm{mH}$ when measured at $1.0 \mathrm{kHz}$. Also, an inductor can be modeled as an inductor in series with a resistor, these resistances were measured along with the inductances on the latter bridge. The resistance associated with $L_{1}$ is $6.72 \Omega$ and the resistance associated with $L_{2}$ is $94.5 \Omega$. Another simulation was constructed which included the updated values for the resistances from the inductors to verify the simulation matched the results. Figure 5.9 shows the results of another WinSpice simulation with superimposed plots depicting the ideal values and the set with the measured inductances and resistances across the inductors. 
The incorrect inductance on $\mathrm{L}_{2}$ with the addition of the large resistance to $\mathrm{L}_{2}$ caused the filter to perform badly.

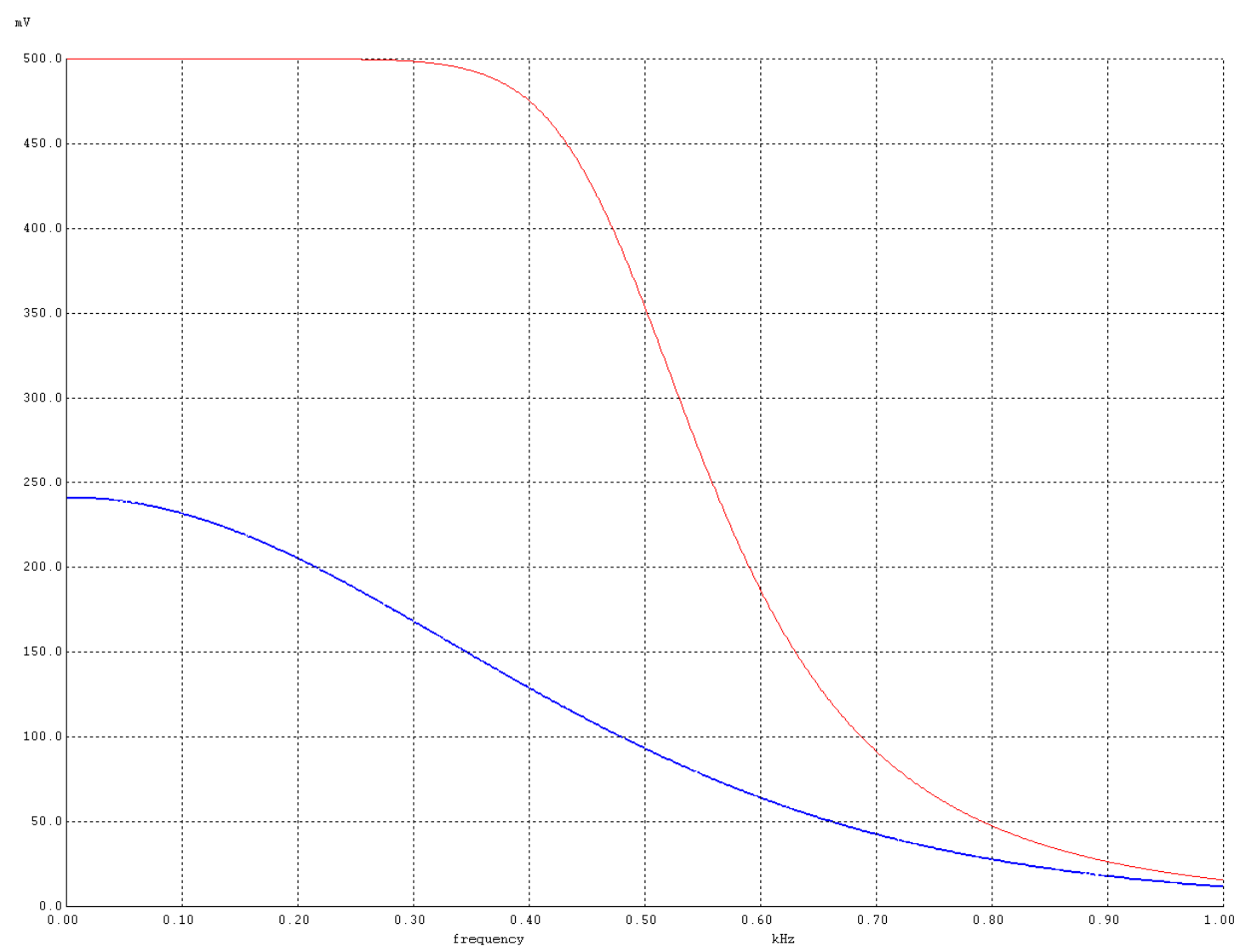

Figure 5.9: Simulated Butterworth frequency response (blue) with updated L-values accounting for resistances. The (red) curve with the overall higher magnitude is the response with the ideal values.

To correct this error, the $30 \mathrm{mH}$ inductor was replaced with three of the $10 \mathrm{mH}$ inductors connected in series, due to the low resistance of the $10 \mathrm{mH}$ inductors and availability of the components in the lab. This reduced the overall resistance to approximately $20 \Omega$. Another simulation and frequency sweep was performed to compare the actual performance of the filter to the simulation. The results of the simulation (Figure 5.10) show that that filter's performance is reduced in both amplitude and cutoff frequency $(360 \mathrm{~Hz})$. 


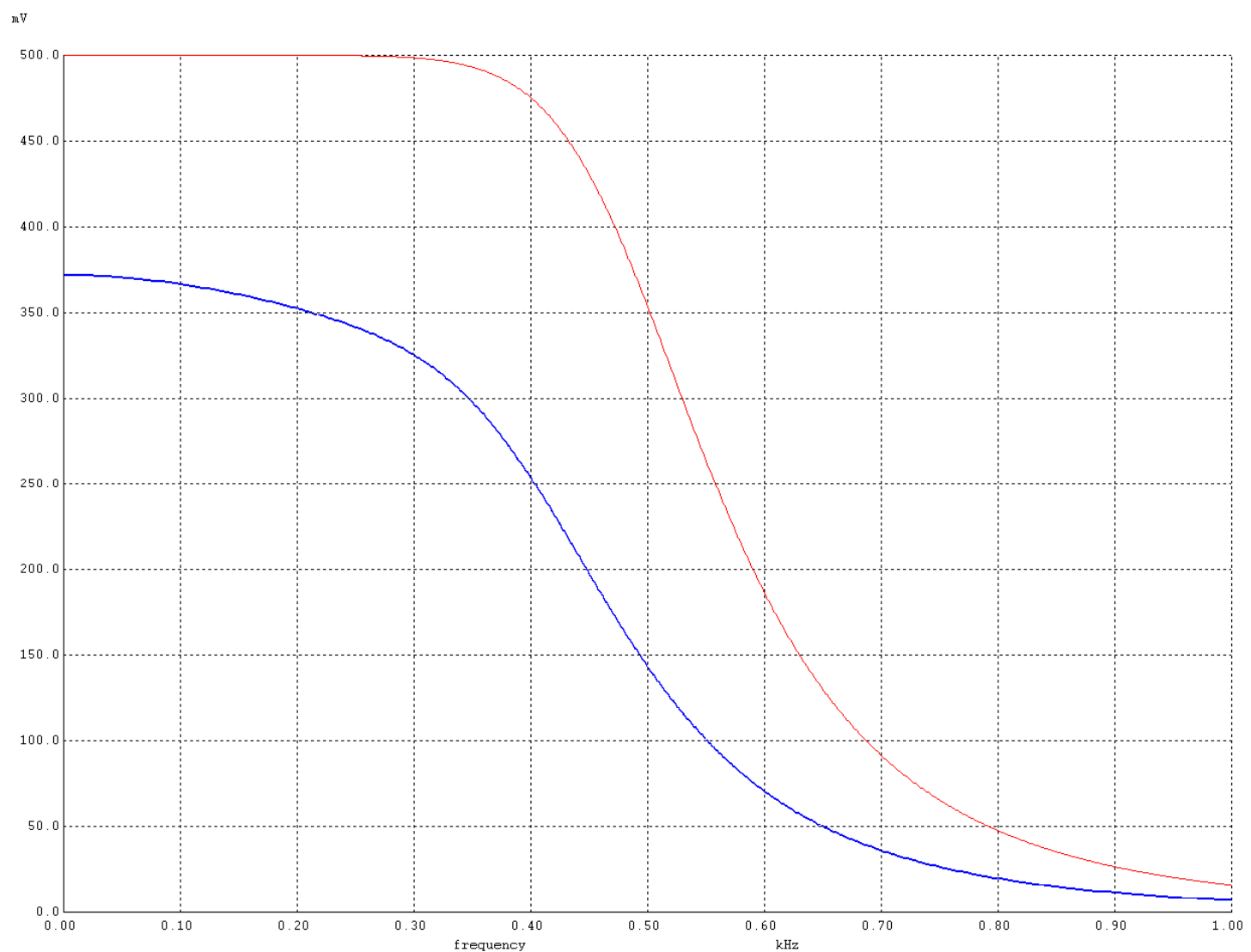

Figure 5.10: Simulated Butterworth frequency response with new L-values (blue). The (red) curve with the overall higher magnitude is the response with the ideal values.

The circuit was tested on the prototyping board with the function generator set to for a linear sweep as in the previous test. The results of this test are displayed in Figure 5.11. The results of this test are similar to the simulation, in that the cutoff frequency is the same as the results from the simulation, although it is less than the desired cutoff frequency. 


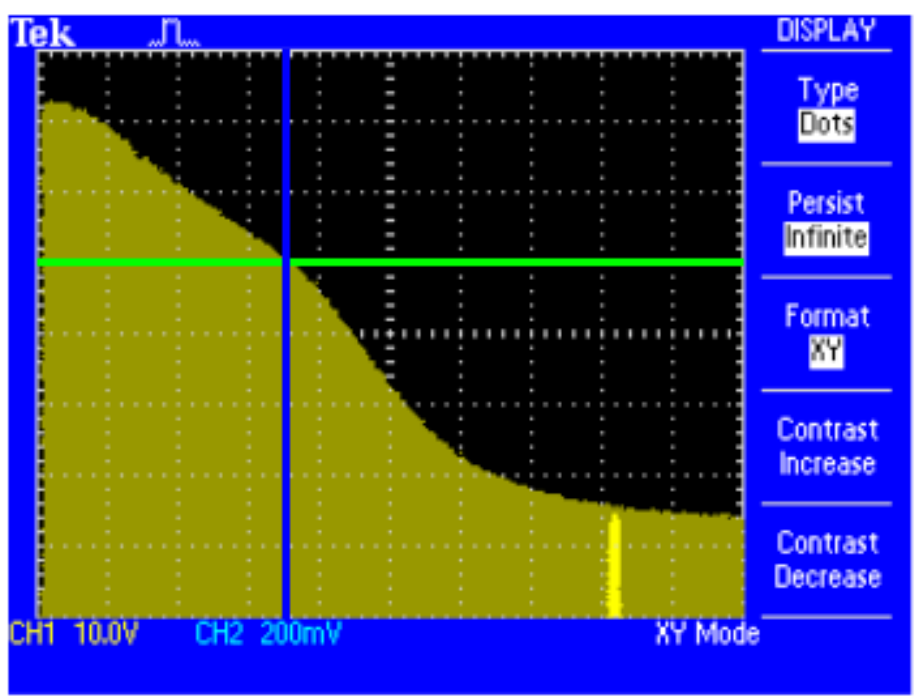

Figure 5.11: Actual Butterworth frequency response using the new L-values with 100Hz/division horizontally and $200 \mathrm{mV} /$ division vertically. The intersecting green and blue lines represent the $3.01 \mathrm{~dB}$ point.

\subsection{Stand-alone Problems}

The ultimate goal of this project is to develop a stand-alone prototype that can run independently of an emulator. From the tests and results in Section 5.3, it is clearly obvious that all the connections and hardware elements work properly with the emulator. The next logical step is to replace the emulator probe on the prototype with the actual microprocessor and monitor the results. Unfortunately, the prototype does not function as expected in stand-alone mode. Therefore, there has to be some hidden flaw in the hardware design or construction that is preventing the 68000 from executing code when it is placed in a socket and set to run independently. This section describes the efforts to resolve this problem.

\subsubsection{Controls and Inputs}

Usually when the emulator is working and the actual processor is not, the problem is that one or more of the unused input lines to the processor are floating. Each of these 
lines (BR', BERR', BGACK', IPL1' and IPL2') was checked with and without the hardware running to see if the lines were floating or connected improperly. Unfortunately, the problem could not be found in these connections. The connections to $\mathrm{V}_{\mathrm{CC}}$, ground $\left(\mathrm{V}_{\mathrm{SS}}\right), \mathrm{HALT}^{\prime}$, RESET', and the system clock were also verified to be working.

\subsubsection{Other Tests}

Testing the load of the various devices was next in the line of logical reasoning. If a device is sourcing or sinking too much current, the device can malfunction in unpredictable ways. The easiest way to test for this on the prototype was to systematically remove parts and attempt to run test code on the emulator first, then on the stand-alone system. However, the MMU and one external interface had to remain in order to know if the system was executing the code. The best interface to leave in place was the MIDI interface because it requires the fewest components to operate. With that in mind, the A/D converter, amplifier, LEDs, data register, SCI ACIA, voltage level shifter, bit rate generator, and sampling frequency generators were removed from the system. Then the MIDI interface test code described was executed to test the functionality. The code ran fine with the emulator connected to the prototype but did not execute with the 68000 . The MIDI interface was removed and the LEDs and data register were replaced to attempt another test. This test used the LED test code as described in Section 5.3.3. The results did not change. Finally, the LEDs and data register were removed and the SCI ACIA, voltage level shifter and bit rate generator were replaced. A simple "Hello World" program was executed and the results were the same as before. 


\subsubsection{MMU Connections}

The next test was to check the various modules for functionality. The first line checked was DTACK' because DTACK' is formed by ANDing the memory select lines. Each time an address is accessed, DTACK' should be asserted. Testing revealed that this was not the case. However, it was proven that the MMU and DTACK' circuitry functioned properly in the previous tests, therefore the problem had to be with the MMU enable lines (AS', UDS', LDS', IACK'/MRST') or data input lines (AB14 - AB16).

To check these lines, DTACK' was forced low so as not to have the 68000 delay instruction execution while waiting for the assertion of DTACK'. For simple memory accesses, asserting DTACK' should pose no problem because the memory operates faster than the 68000. Probing AS', UDS' and LDS' revealed something interesting, in that each of these signals on the oscilloscope appeared to be functioning as a clock signal, or pulse train. Normally, the variety of commands associated with sending/receiving data cause delays in the assertion/negation of these lines, which never cause these lines to appear as a pulse train. However, it was easy to verify that these signals were reaching the MMU by probing the base of the MMU and the top of the MMU.

This meant that the address lines had to be causing the problem. Probing the tops of the pins on the 68000 revealed that the address lines were working properly, but the signals were not reaching the MMU. This was puzzling because the emulator worked fine and the continuity of the connections was verified earlier. On a hunch, the base of the sockets housing the 68000 was probed. This revealed that MMU data input lines were floating, meaning there had to be a mechanical connection problem. This is not too surprising because the 68000's pin diameters are smaller than the pin diameters on the 
emulator pod probe. When inserted into the zero insertion force (ZIF) socket, the emulator connections are more secure. The ZIF socket itself sits in a machined socket, so it is no problem to remove the ZIF socket and insert the 68000 into the machined socket when ready to execute the code stand-alone. Additionally, all other mechanical connections were examined for inconsistencies, but no others were identified.

Once this problem was identified, the proven code (The Game of Snake) was loaded and executed again. This time the code executed up to a certain point with the 68000 in place, but the 68000 locked up. This problem occurred at the same place in the code every time, and the error was independent of the duration of code execution before the error occurred. This suggested there was a problem in the memory space, although that would be unlikely because the memory works fine with the emulator. The next step was to repeat the tests from Section 5.4 .2 before proceeding.

\subsubsection{Checking the Memory Space}

The results from performing the tests in Section 5.4.2 again were different from the first time around. In this instance, there were mixed results for the smaller code segments. In some segments, such as the LED test, the code executed correctly. In other tests, such as the interrupt request test and the MIDI interface test, the code would not execute correctly. When the proven Game of Snake code was executed, the problems were the same as before.

Essentially, these results made no sense. First, the failures seemed to be independent of memory location, which would suggest a problem with the address line or the memory itself. Second, the failures seemed to be independent of the device operated. For example, in the smaller test segments, the SCI ACIA would not output a "Hello 
world" message to the PC terminal, but the ACIA handled I/O correctly for the Game of Snake, which makes extensive use of the ACIA.

As stated in the previous section, the proven code always locked up in the same location in the game, although the address present on the 68000 was neither in the program nor the data space for this design. However, a test was performed to see if the error would follow the code or stay fixed to the address region where the error was occurring. Without knowing the exact location of the address, the test was performed by a number of no-op (NOP) statements at the beginning of the code. This essentially had the effect of pushing the remaining code down by two bytes for each NOP statement. Up to 20 consecutive NOP statements were added to perform this test, but the error followed the code each time. This once again made no sense because the code has been proven to work on a previous design as well as with the emulator connected to the prototype. Aside from these difficulties, this consistently reproducible error ceased to be consistent, and the code executed intermittently.

\subsubsection{Testing Conclusions}

These problems were analyzed, researched, and tested for months with no measurable success. The inability to consistently reproduce the errors has made debugging impossible without the ability to use the emulator. Ideally, a 32-bit logic analyzer could help in determining where the 68000 fails to execute code, but since the errors are independent of memory addresses and device, this expensive tool would prove useless. The problem itself likely comes from a problem with the soldering, noise imposed by the $8 \mathrm{MHz}$ clock, and/or from a slightly excessive current draw. All of these potential problems have been tested as described in the previous sections, but no 
conclusive results have been obtained. Therefore, the final design will have to run on the emulator as the cost associated with trying to fix this error far outweighs any benefit for this project. 


\section{CHAPTER VI - CONCLUSIONS AND FUTURE WORK}

The AMDF time-domain analysis technique was chosen to aid in real-time pitchtracking for a voice operated musical instrument operated by the MIDI protocol. The AMDF algorithm was implemented in assembly to run on an embedded system utilizing the 68000 , which was constructed on a soldered prototype board. This design consisted of four main hardware modules responsible for controlling sampling, serial communications, MIDI communications and memory management. Although the modules worked correctly individually and together with an emulator, they failed to function on a stand-alone prototype due to an unidentifiable hardware flaw.

However, the simulations and physical implementation clearly revealed that the AMDF by without additional refinements itself is insufficient to function accurately as a real-time pitch-tracking device with such a small sampling rate. Additional software refinements have been proven to enhance the functionality of the AMDF for this application, but were not implemented due to limitations imposed by the system clock. Thus, equipment limitations leading to hardware design constraints were a factor for reduced performance of this device, as well as a limiting factor for incorporating additional refinements to support the AMDF, as implemented by [Shimamura 2001 and Kim 1998].

Further work should be done to find a suitable minimum acceptable system clock capable of supporting a modified AMDF or another successful pitch detection algorithm running on an embedded system. A study similar to [Rabiner 1976] updated to include more frequency and time domain techniques including a variety of the updated FFT algorithms and zero-crossing techniques would be extremely beneficial in this field, 
especially if the study were a comparison of real-time performance on modern embedded hardware.

This prototype was designed to perform all signal calculations itself. Another obvious area of future work would be to implement this design using an external DSP device or an external programmable logic device using VHDL or ABEL. These design practices are implemented in industry especially when dealing with RF systems, where generic or specially designed ICs are used to modulate, demodulate, or process these high-frequency signals. Either of these concepts would alleviate computational-related performance problems on the main processor. However, these devices would likely require a system clock faster than the $8 \mathrm{MHz}$ clock in this design. Either implementation would lead to a significantly more successful design of an embedded real-time voice operated musical instrument.

Ultimately, the success of a device of this nature rests with a faster system clock and a faster processor or microcontroller. In conjunction with a faster system, using an external device to handle the signal processing aspect of this application would lead to a more successful prototype and marketable product. 


\section{REFERENCES}

ANSI (1997). ANSI S1.1-1994: American national standard acoustical terminology. Acoustical Society of America, New York, 1994.

Budak, A. (1991). Passive and Active Network Analysis and Synthesis. Prospect Heights, Illinois: Waveland Press, Inc. Copyright 1974. Republished 1991.

Cleaver, T.G. (2000). Voice Operated Musical Instrument. MEng/MS Thesis Opportunity. January 10, 2000.

Cleaver, T.G. (2004). Needs Assessment. 2004. Accessed 27 Nov. 2007. $<$ http://raise.spd.uofl.edu/systom/ece599/NeedsAssessment.pps $>$

de Cheveigné, A. and Kawahara, H. (1998). Multiple period estimation and pitch perception model. Speech Communications Vol. 27, pp. 175-185. Elsevier Science B.V. July 8, 1998.

de Cheveigné, A. and Kawahara, H. (2002). YIN, a fundamental frequency estimator for speech and music. Journal of the Acoustical Society of America, Vol. 111, No. 4, pp. 1917-1930, April 2002.

de la Cuadra, P., Master, A., Sapp, C. (2001). Efficient Pitch Detection Techniques for Interactive Music. Proceedings of the 2001 International Computer Music Conference, La Habana, Cuba, September 2001.

“diplophonic.” Dictionary.com Unabridged (v 1.1). Random House, Inc. Accessed 25 Jul. 2007. <Dictionary.com http://dictionary.reference.com/browse/diplophonic>.

"Fast Fourier Transform." [FFT]. Wikipedia. 2007. Wikimedia Foundation, Inc. Accessed 14 July 2007. <http://en.wikipedia.org/wiki/Fast_Fourier transform>.

"formant." The American Heritage ${ }^{\circledR}$ Dictionary of the English Language, Fourth Edition. Houghton Mifflin Company, 2004. Accessed 27 Nov. 2007. <Dictionary.com http://dictionary.reference.com/browse/formant>.

“glottis.” Merriam-Webster's Medical Dictionary. Merriam-Webster, Inc. 25 Jul. 2007. $<$ Dictionary.com http://dictionary.reference.com/browse/glottis $>$.

Lago, N.P and Kon, F. (2004). The Quest for Low Latency. Proceedings of the International Computer Music Conference, 2004. pp. 33-36.

Middleton, G. Pitch Detection Algorithms. 17 Dec. 2003. Rice University. 27 January 2006. < http://cnx.rice.edu/content/m11714/latest/>. 
MIDI Manufacturers Association (MMA). Making Music with MIDI. Copyright 19852007. Accessed 10 Jan. 2007. <http://www.midi.org/aboutmidi/aboutmidi3.shtml>.

MIDI Manufacturers Association (MMA). MIDI Electrical Specification Diagram \& Proper Design of Joystick/MIDI Adapters. Copyright 1985. Accessed 9 July 2007. <http://www.midi.org/about-midi/electrical.shtml $>$.

MIDI Manufacturers Association (MMA). Table 1 of 3 - Summary of MIDI Messages. Updated 1995. Accessed 9 July 2007. <http://www.midi.org/aboutmidi/table1.shtml>.

MIDI Manufacturers Association (MMA). Table 2 of 3 - Expanded Messages List (Status Bytes). Updated 1995. Accessed 9 July 2007.

$<$ http://www.midi.org/about-midi/table2.shtml $>$.

MIDI Manufacturers Association (MMA). Tutorial on MIDI and Music Synthesis. Revised 2001. Accessed 9 July 2007. <http://www.midi.org/aboutmidi/tutorial/tutor.shtml>.

Motorola Inc. (1993). M68000 8-/16-/32-Bit Microprocessors User’s Manual. Ninth Edition. Copyright 1993.

Oppenheim, A.V. and Schafer, R.W. (1999). Discrete-Time Signal Processing. Saddle River, New Jersey: Prentice-Hall Inc. Copyright 1999.

“psychoacoustics.” Merriam-Webster's Medical Dictionary. Merriam-Webster, Inc. Accessed 25 Jul. 2007. <Dictionary.com http://dictionary.reference.com/browse/psychoacoustics $>$.

Rabiner, L.A., Cheng, M.J., Rosenberg, A.E., \& McGonegal, C.A. (1976). A Comparative Performance Study of SeveralPitch Detection Algorithms. IEEE Transactions on Acoustics, Speech and Signal Processing, Vol. ASSP-24, No. 5, October 1976, pp. 399-418.

Ryynänen, M. (2004). "Probabilistic Modeling of Note Events in the Transcription of Monophonic Melodies". MS thesis. Tampere University of Technology, Department of Information technology, February 11, 2004.

Saul, L.K., Lee D. D., Isbell. C.L., \& LeCun, Y. (2002). Real time voice processing with audiovisual feedback: toward autonomous agents with perfect pitch. Advances in Neural Information Processing Systems 15 (NIPS) Conference Paper, December 9, 2002, pp 1205-1212.

Semenov, O. PC-MIDI interface adapter (MIDI Cable) schematic and pinout. 25 June 2005. Accessed 5 July 2007. <http://www.pinouts.ru $>$. 
Shimamura, T. \& Kobayashi, H. (2001). Weighted Autocorrelation for Pitch Extraction of Noisy Speech. IEEE Transactions on Speech and Audio Processing, Vol. 9, No. 7, October 2001, pp. 727-730.

Stewart, L. "Listening Displeasure". BBC News Magazine. 30 Jan. 2006. Accessed 3 July 2007. $<\underline{\text { http: } / / \text { news.bbc.co.uk/1/hi/magazine/4655352.stm> }}$.

Yale University Music Library. Vocal Ranges. March 21, 2005. Accessed 15 Oct. 2006. $<\underline{\text { http://www.library.yale.edu/cataloging/music/vocalrg.htm }>\text {. }}$

Weeks, M. (2007). Digital Signal Processing Using MATLAB and Wavelets. Hingham, Massachusetts: Infinity Science Press. Copyright 2007. 


\section{APPENDIX I - HARDWARE DESIGN \\ Schematic Page 1}

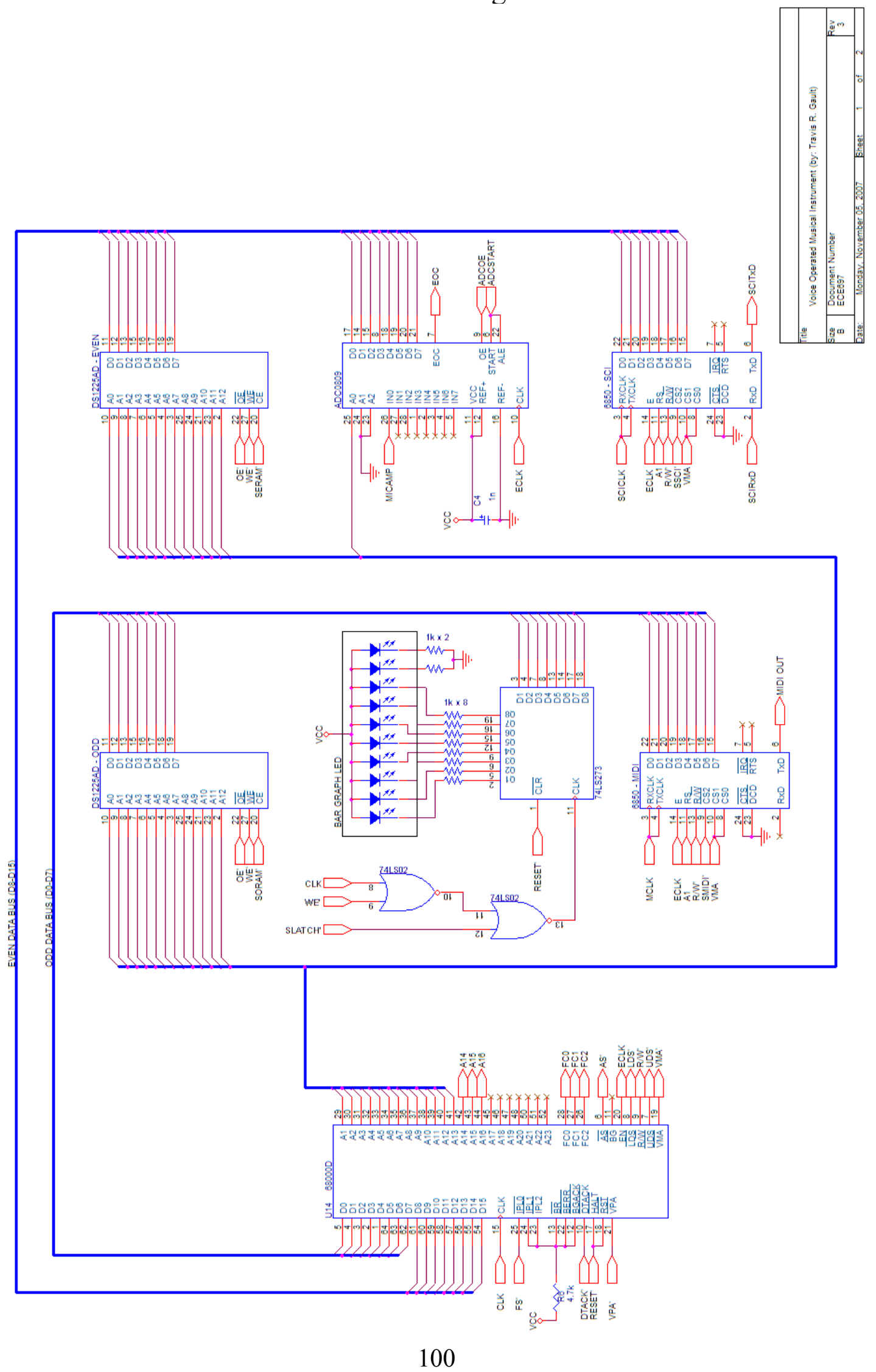




\section{Schematic Page 2}
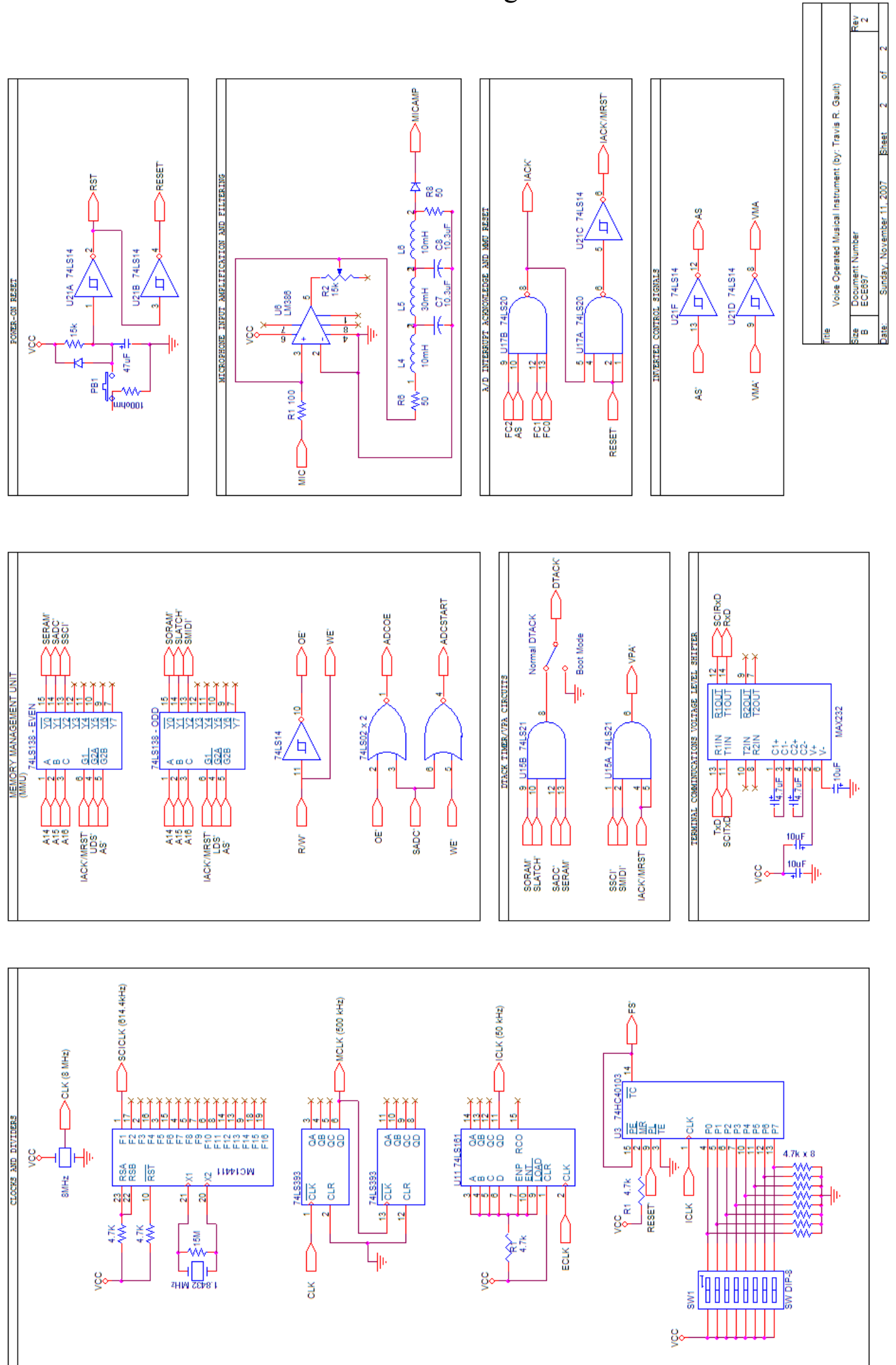
Board Layout - Top View of All Components

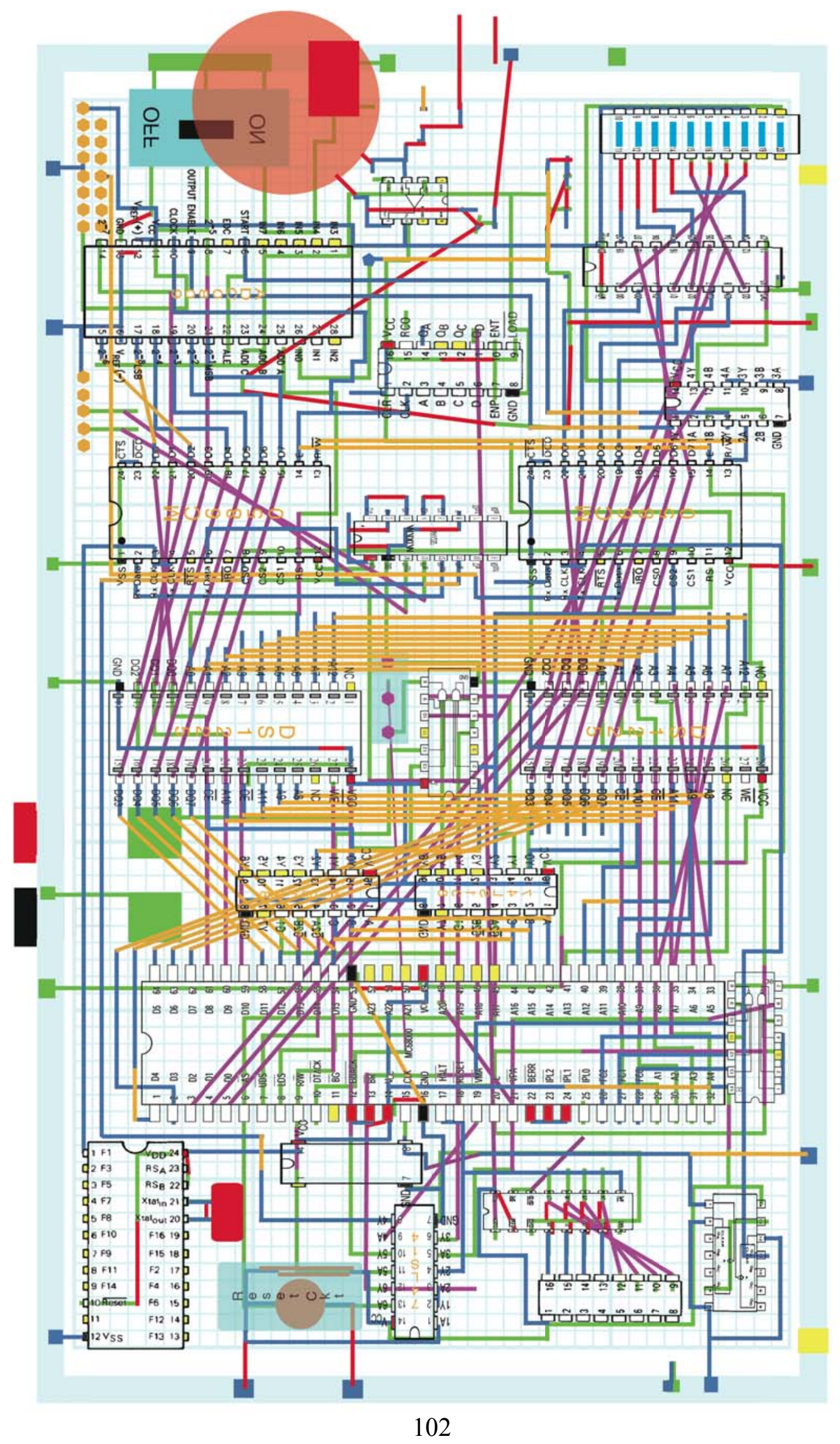




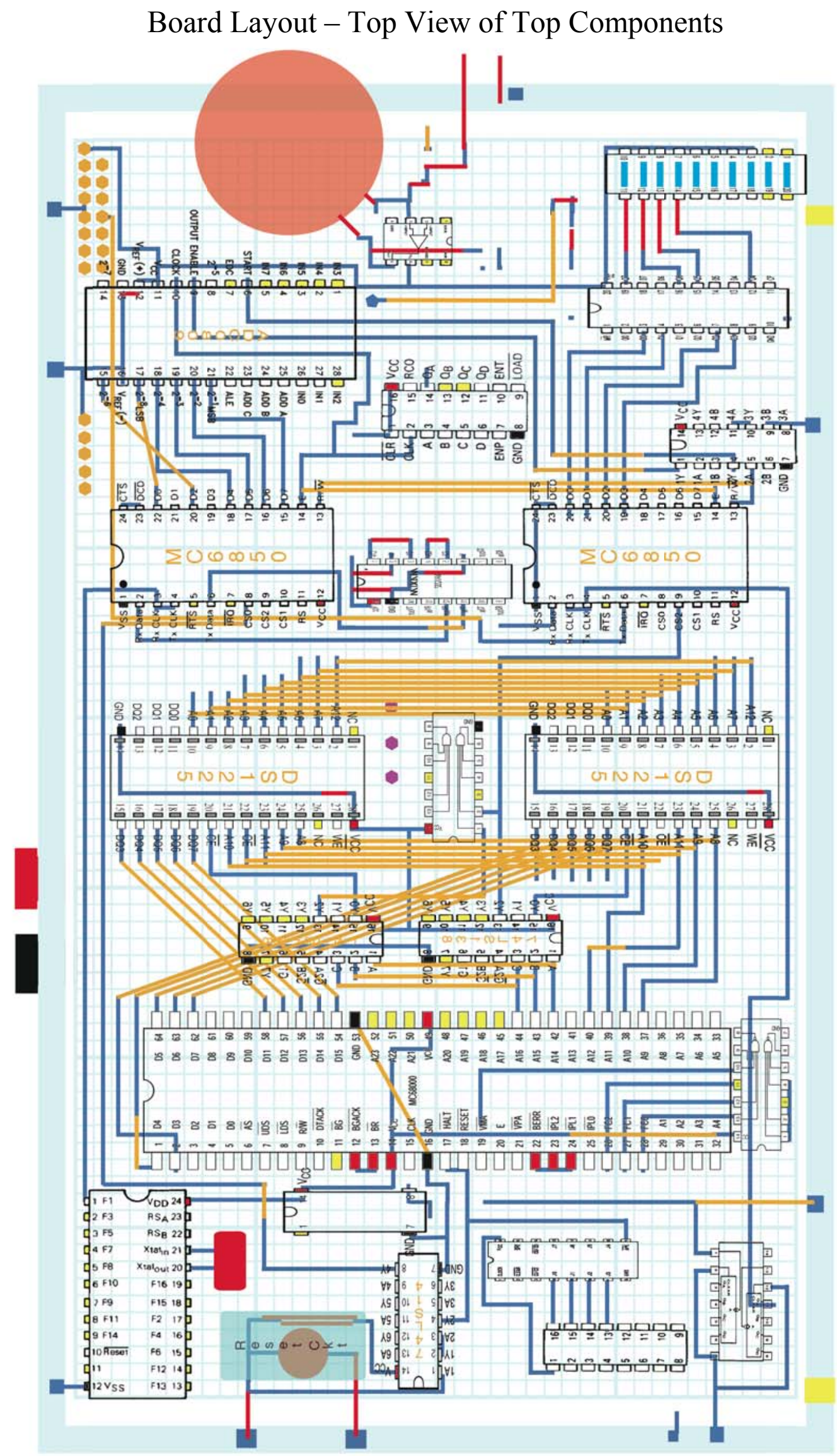




\section{Board Layout - Bottom View of Bottom Components}

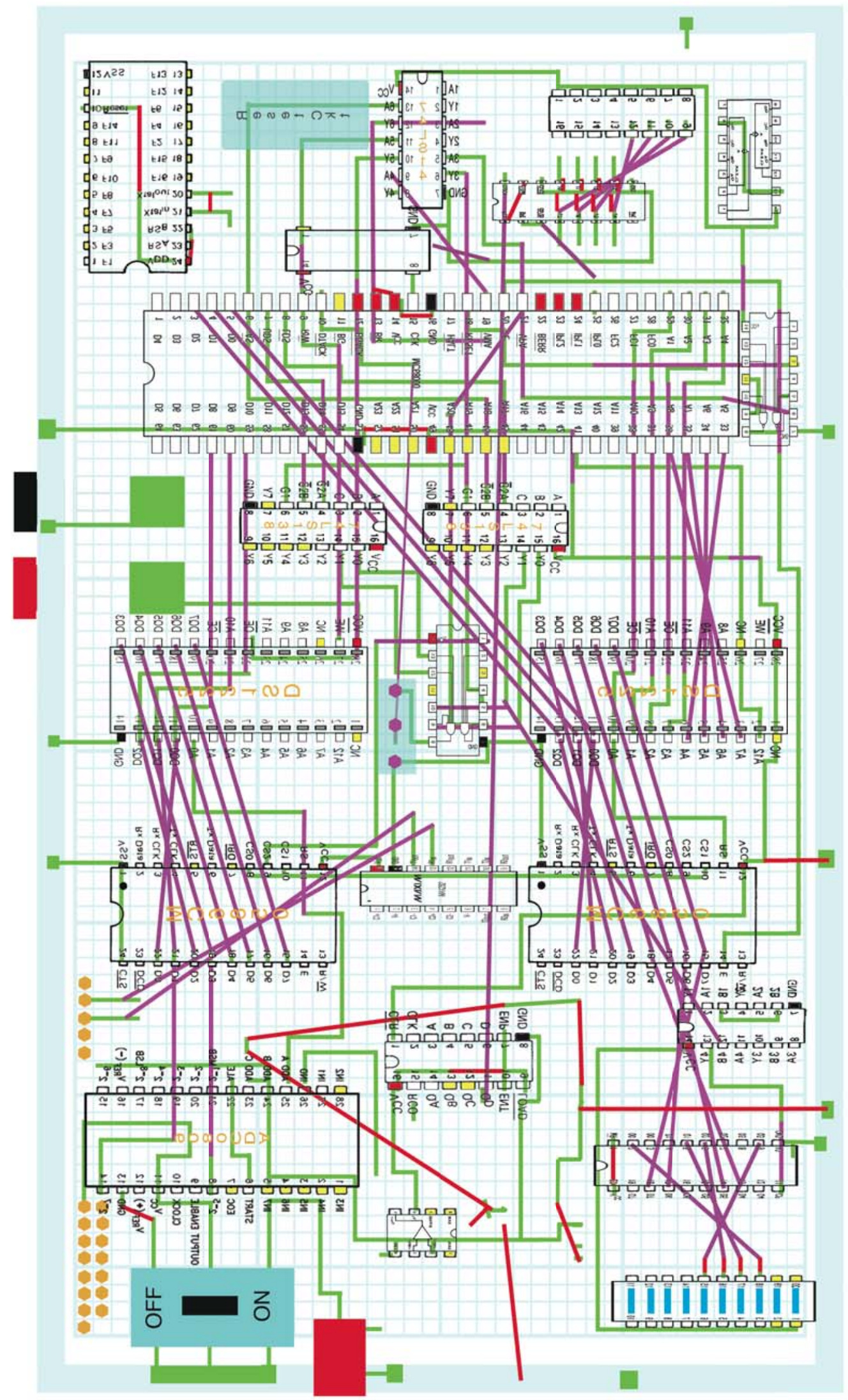




\section{APPENDIX II - SIMULATION SOURCE CODE}

\section{Butterworth Filter Spice Simulation File for Ideal Component Values}

(BWLPF.spc)

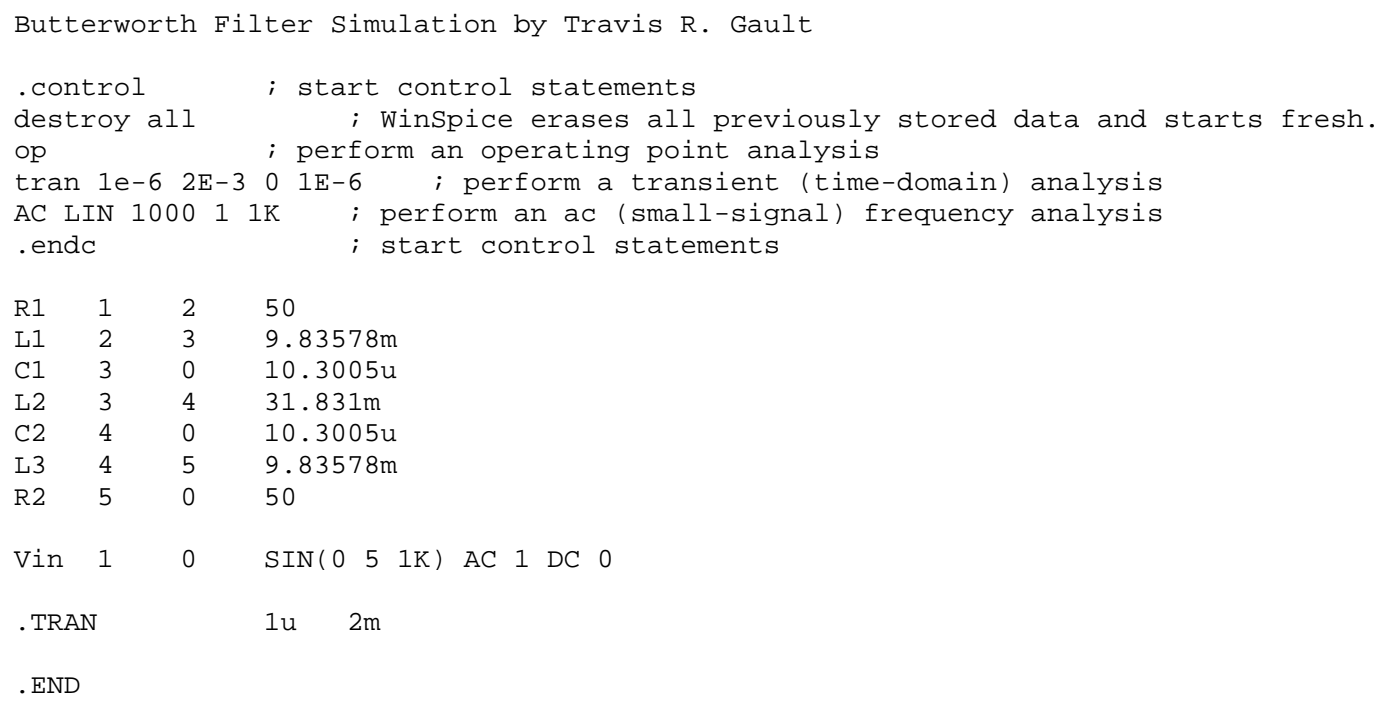

Butterworth Filter Spice Simulation File for Actual Component Values with Updated Inductance and Resistance Values (BWLPF-Actual Values with LR.spc) 


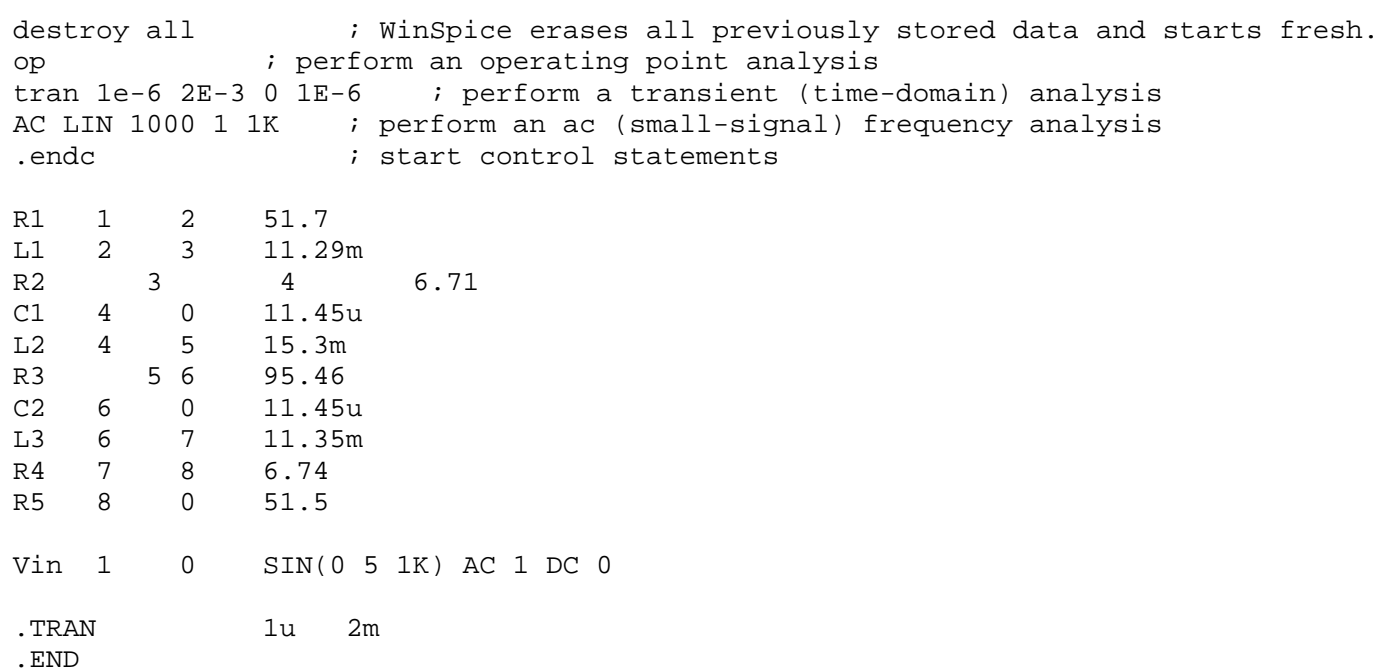

\section{Butterworth Filter Spice Simulation File for Actual Component Values with New Inductance and Resistance Values (BWLPF-Actual Values with new LR.spc)}

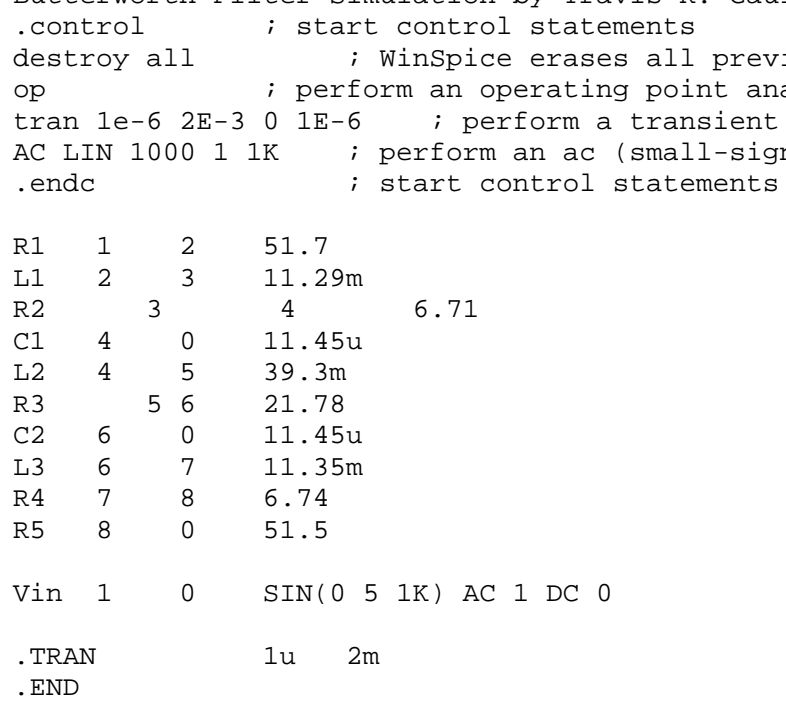




\section{AMDF MATLAB Simulation File (amdfTests.m)}

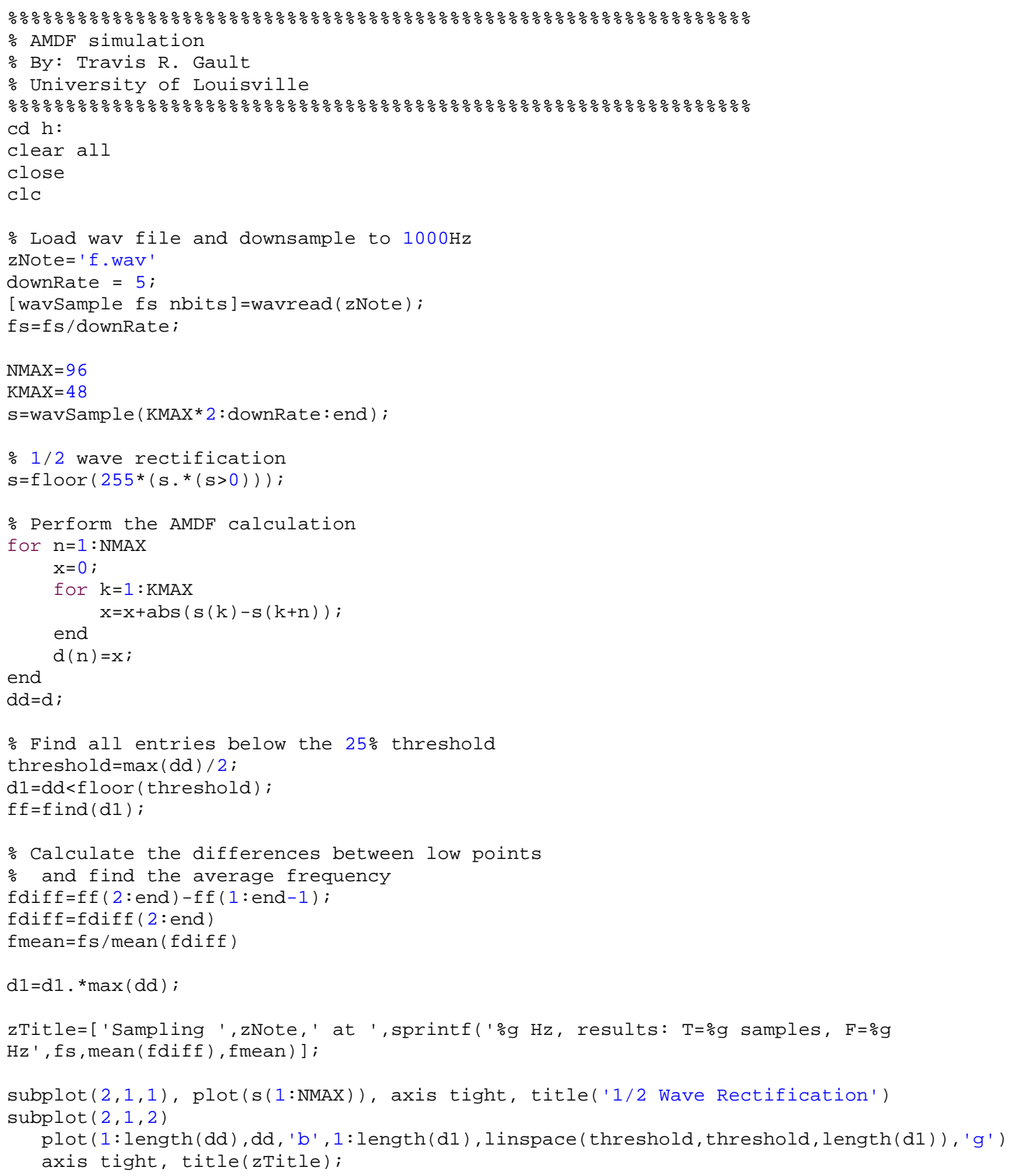




\section{APPENDIX III - MODULE TEST CODE}

\section{LEDTest.x68}

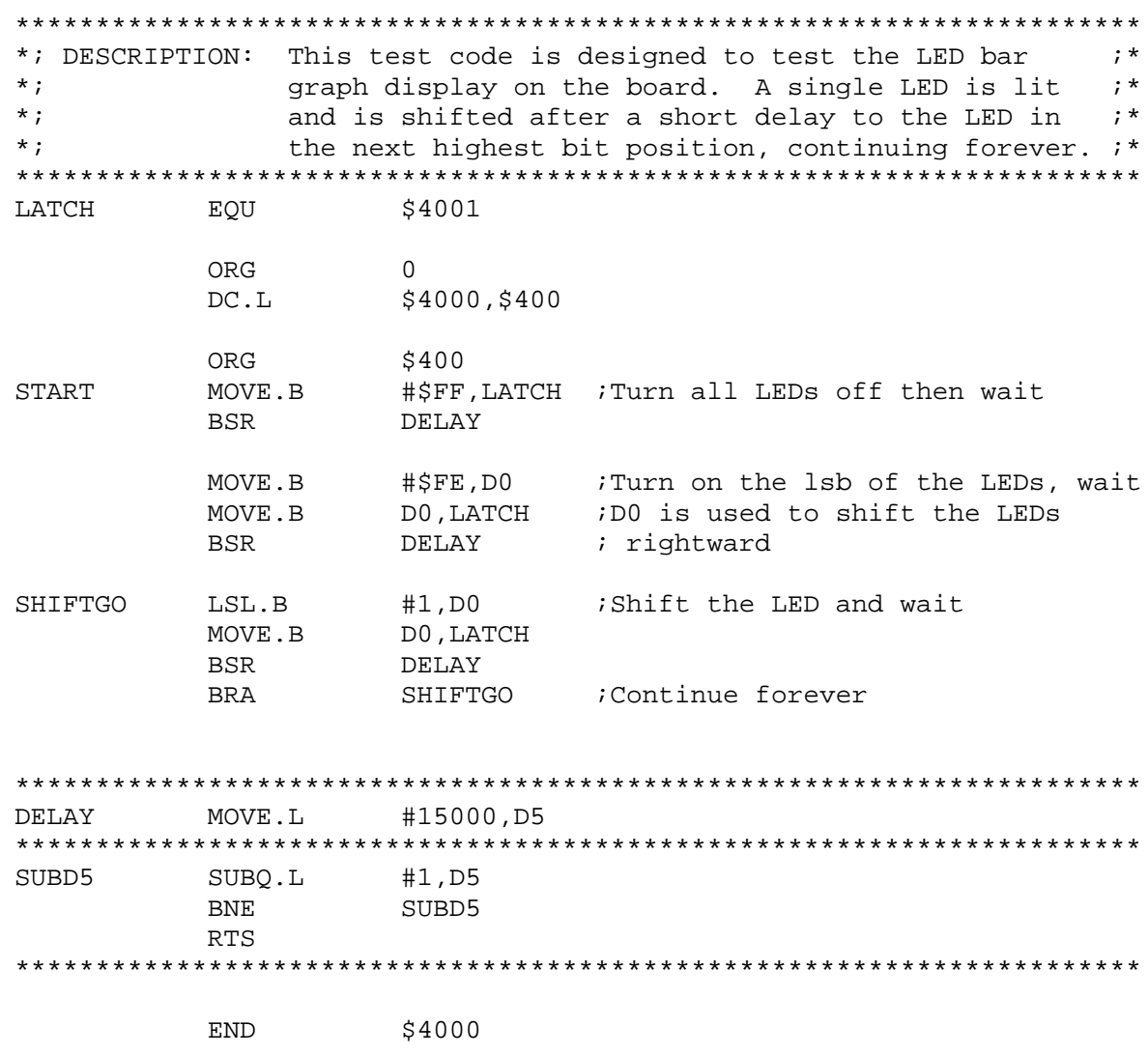




\section{MIDItest.x68}

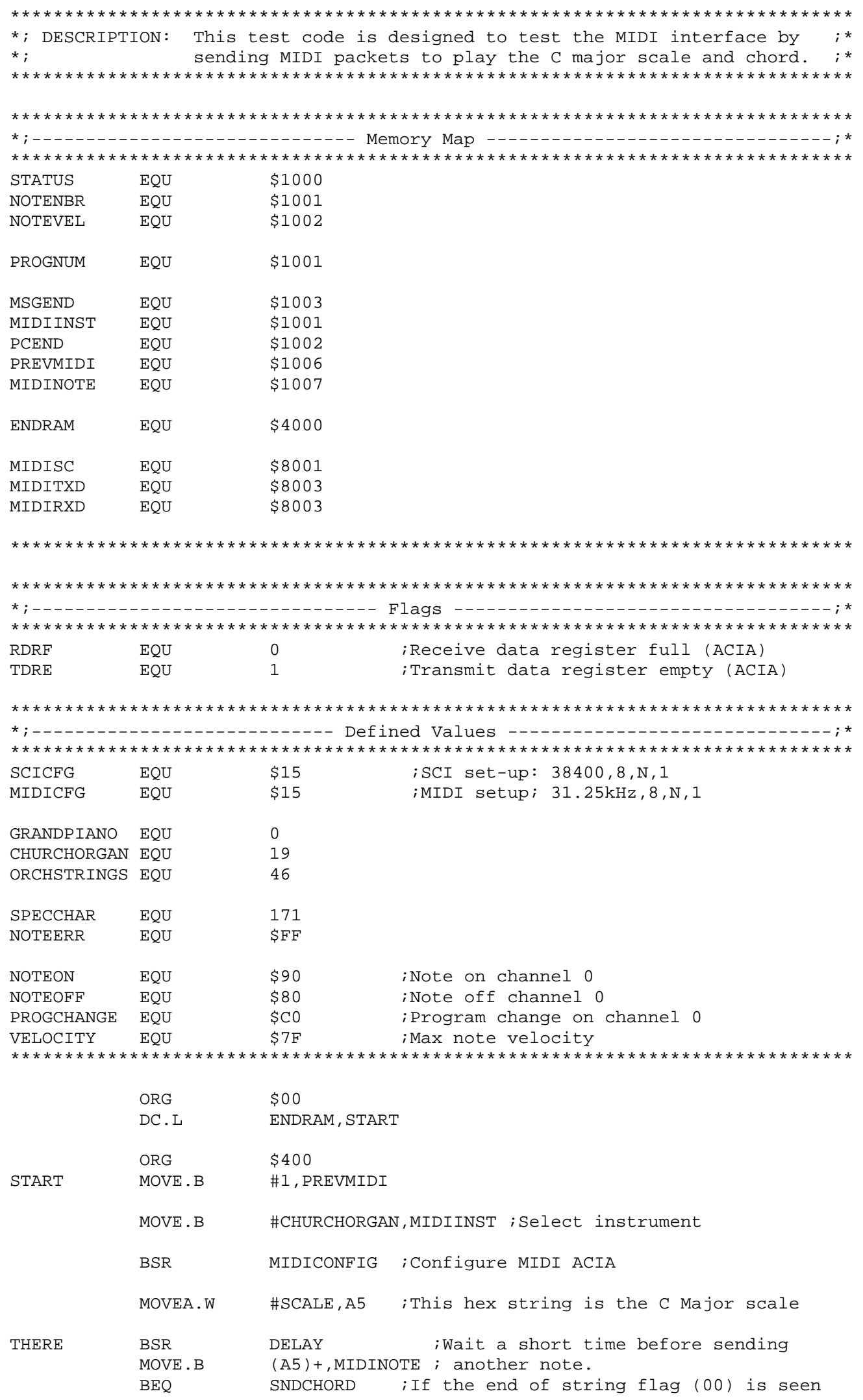




\begin{tabular}{|c|c|c|}
\hline & $\begin{array}{l}\text { BSR } \\
\text { BRA }\end{array}$ & $\begin{array}{ll}\text { SENDMIDI } & ; \text { then send the I chord } \\
\text { THERE } & ; \text { Else, send the next note in the scale }\end{array}$ \\
\hline \multirow[t]{2}{*}{ SNDCHORD } & MOVEA.W & ; This hex string is the $\mathrm{C}$ major chord \\
\hline & $\begin{array}{l}\text { MOVE.B } \\
\text { MOVE.W }\end{array}$ & $\begin{array}{l}\text { \#NOTEON, STATUS } \\
\text { \#STATUS, MIDISTR }\end{array}$ \\
\hline \multirow[t]{3}{*}{ CNOTES } & $\begin{array}{l}\text { MOVE. B } \\
\text { BEQ }\end{array}$ & $\begin{array}{ll}\text { (A5)+, NOTENBR } & \text {; Send each not in the chord, no delay } \\
\text { CHORDHOLD } & \text {; Wait a while after all note are send }\end{array}$ \\
\hline & BSR & MIDISend \\
\hline & BRA & CNOTES \\
\hline \multirow[t]{2}{*}{ CHORDHOLD } & BSR & ; Play the chord fora while \\
\hline & BSR & DELAY \\
\hline \multirow[t]{3}{*}{ SILENCE } & MOVEA.W & \#CHORD, A5 ; Silence the notes \\
\hline & MOVE.B & \#NOTEOFF, STATUS \\
\hline & MOVE.W & \#STATUS, MIDISTR \\
\hline \multirow[t]{4}{*}{ NOTESOFF } & MOVE.B & $($ A5 ) + , NOTENBR \\
\hline & BSR & MIDISend \\
\hline & BSR & MINIDELAY \\
\hline & BRA & NOTESOFF \\
\hline \multirow[t]{2}{*}{ DONE } & STOP & ;Stop execution \\
\hline & BRA & DONE \\
\hline \multicolumn{3}{|c|}{ 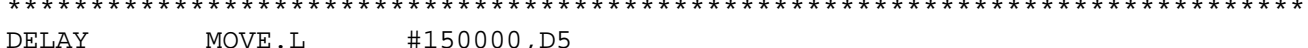 } \\
\hline \multicolumn{2}{|c|}{$\begin{array}{l}\text { DELAY MOVE. L } \\
* * * * * * * * * * * * * * * * * *\end{array}$} & 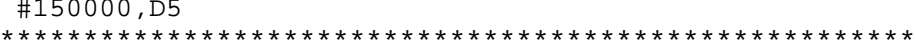 \\
\hline \multirow[t]{3}{*}{ SUBD5 } & SUBQ. L & $\# 1$, D5 \\
\hline & BNE & SUBD5 \\
\hline & RTS & \\
\hline \multicolumn{3}{|c|}{ 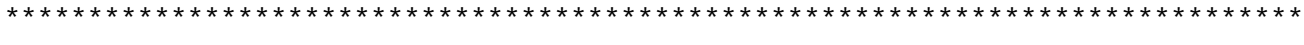 } \\
\hline \multirow{4}{*}{$\begin{array}{l}\text { MINIDELAY } \\
\text { SUBD }\end{array}$} & MOVE.W & \#150, D5 \\
\hline & SUBQ.W & $\# 1$, D5 \\
\hline & BNE & SUBD \\
\hline & RTS & \\
\hline \multicolumn{3}{|c|}{ 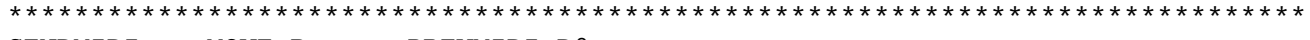 } \\
\hline \multirow[t]{16}{*}{$\begin{array}{l}\text { SENDMIDI } \\
* * * * * * * * * *\end{array}$} & $\begin{array}{l}\text { MOVE. B } \\
* * * * * * *\end{array}$ & $\begin{array}{l}\text { PREVMIDI, DO } \\
* * * * * * * * * * * * * * * * * * * * * * *\end{array}$ \\
\hline & MOVE.B & MIDINOTE,D1 ; If the previous note and current note \\
\hline & CMP.B & D0,D1 ; are the same, there is no need to send \\
\hline & BEQ & DONESNDMIDI ; other MIDI packets, so exit \\
\hline & MOVE.B & ;Else, silence the previous note \\
\hline & MOVE.B & PREVMIDI, NOTENBR \\
\hline & MOVE.B & \#VELOCITY, NOTEVEL \\
\hline & MOVE.B & $\# \odot$, MSGEND \\
\hline & MOVE.W & \#STATUS, MIDISTR \\
\hline & BSR & MIDISend \\
\hline & CMPI.B & \#NOTEERR,D1 ; If there was an error identifying $f \odot$, or \\
\hline & BEQ & DONESNDMIDI ; the output needs to be silent, exit \\
\hline & MOVE.B & ; Else send the new note and exit \\
\hline & MOVE.B & MIDINOTE, NOTENBR \\
\hline & MOVE.B & D1, PREVMIDI ; Now the current note is the old one \\
\hline & BSR & MIDISend \\
\hline & RTS & \\
\hline
\end{tabular}




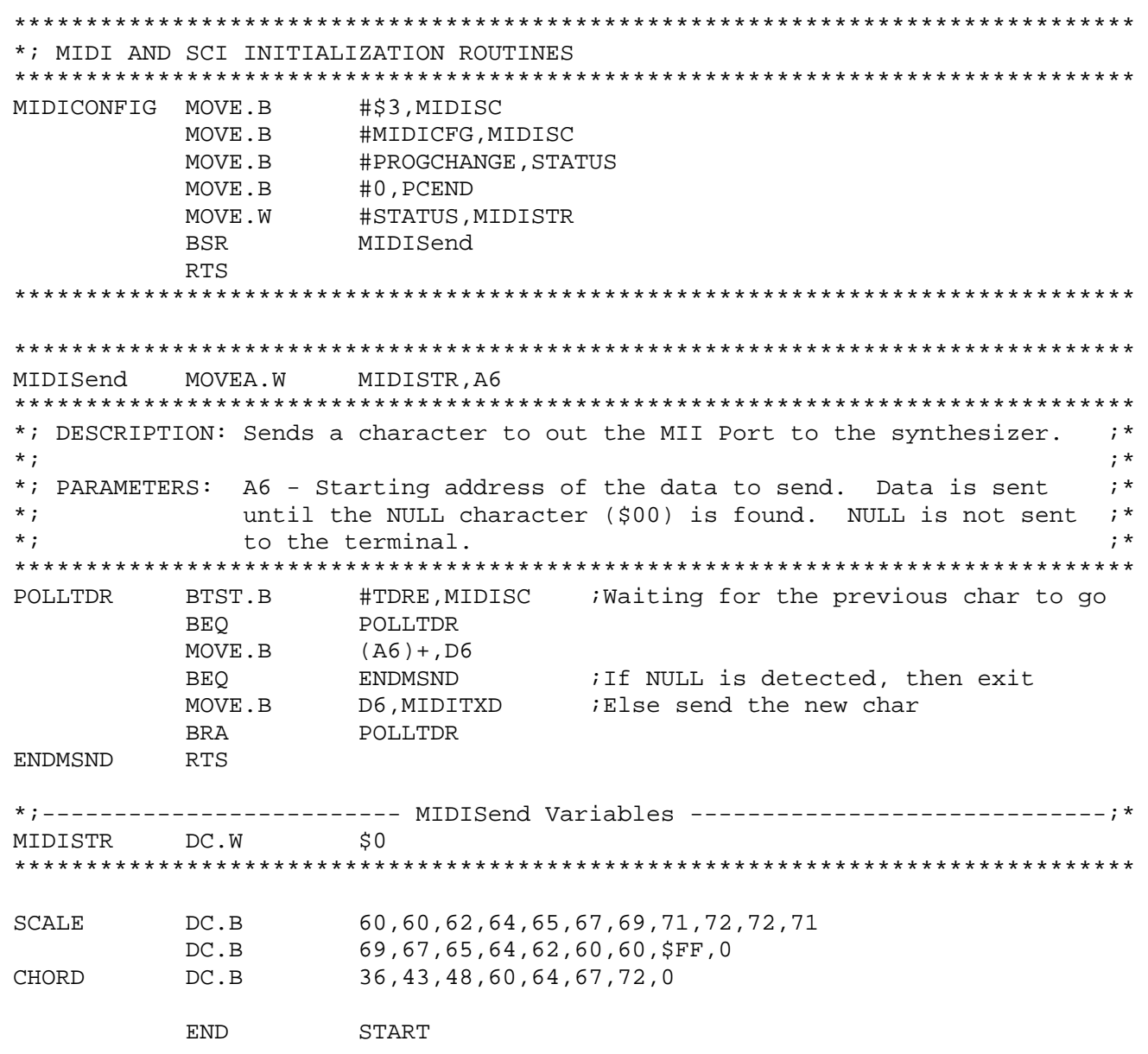

\section{IRQtest.x68}

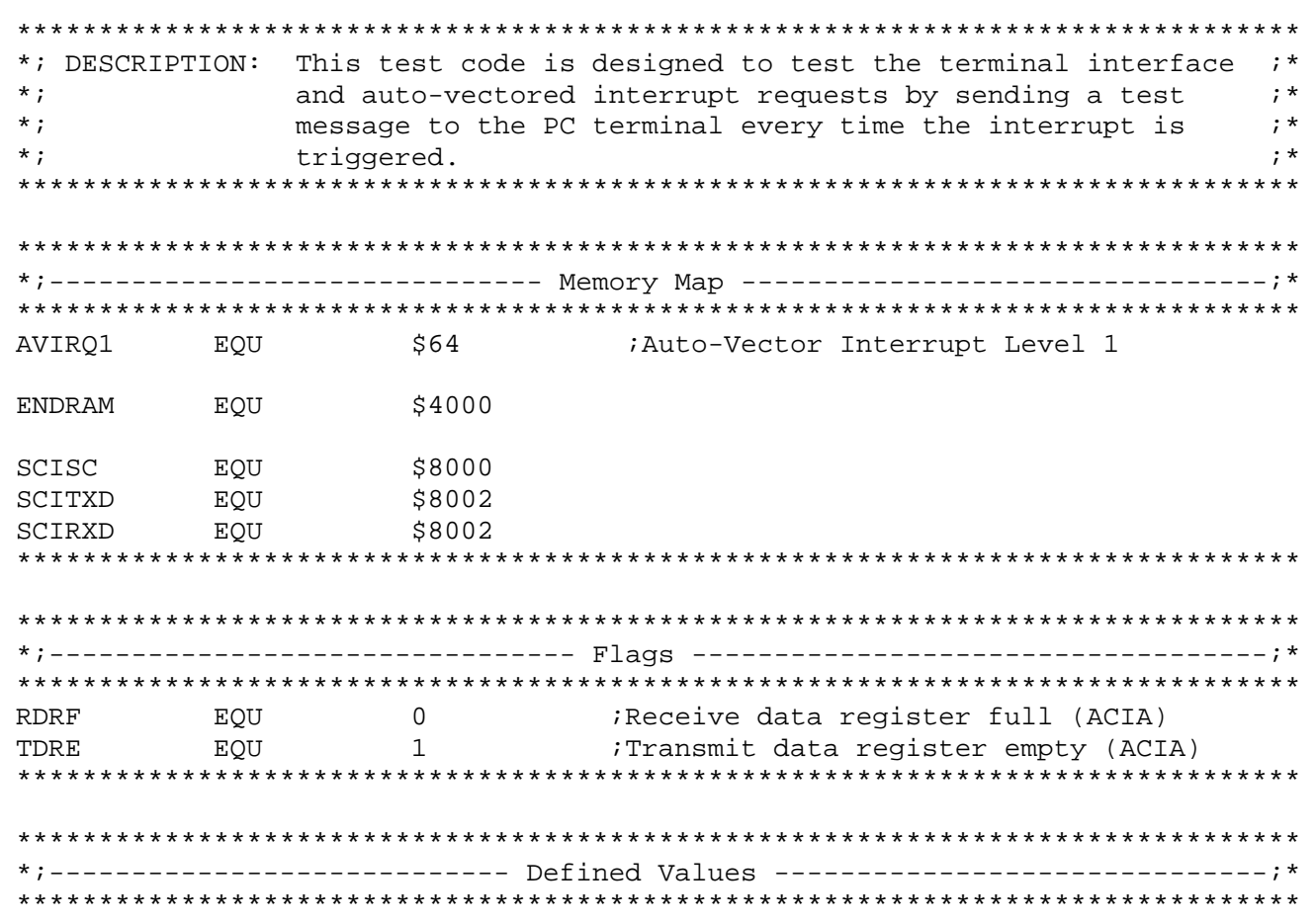




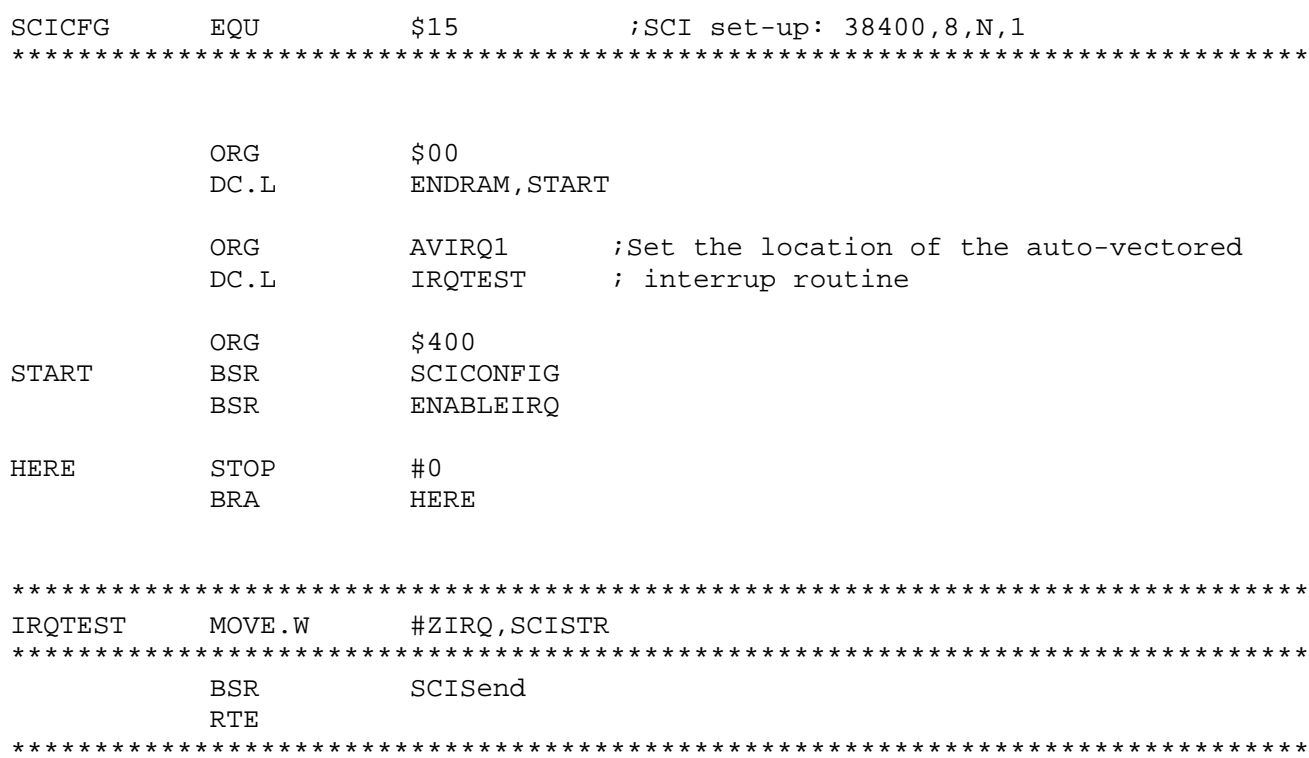

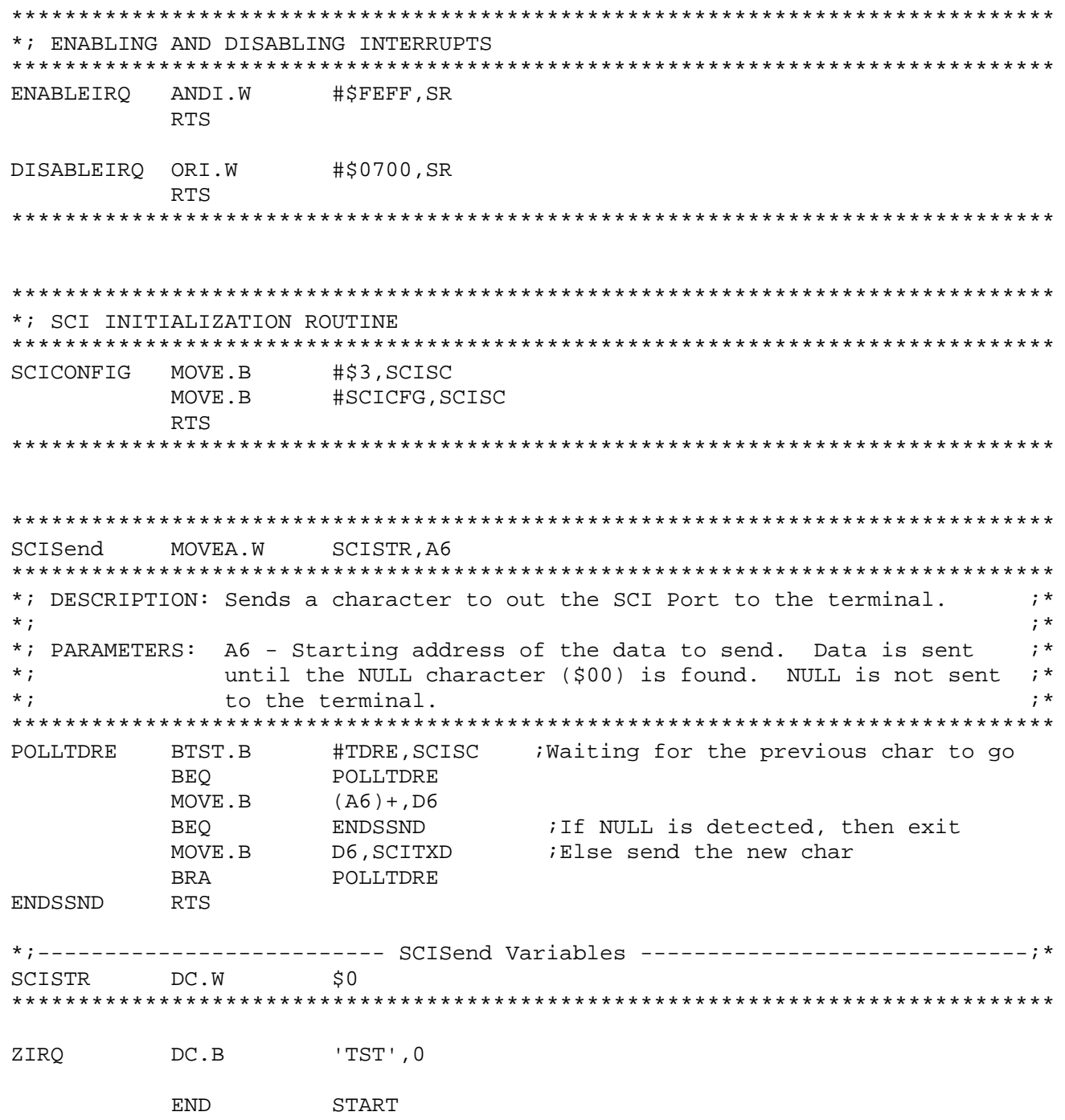




\section{The Proven Code for the Game of Snake (nibblesB.x68)}

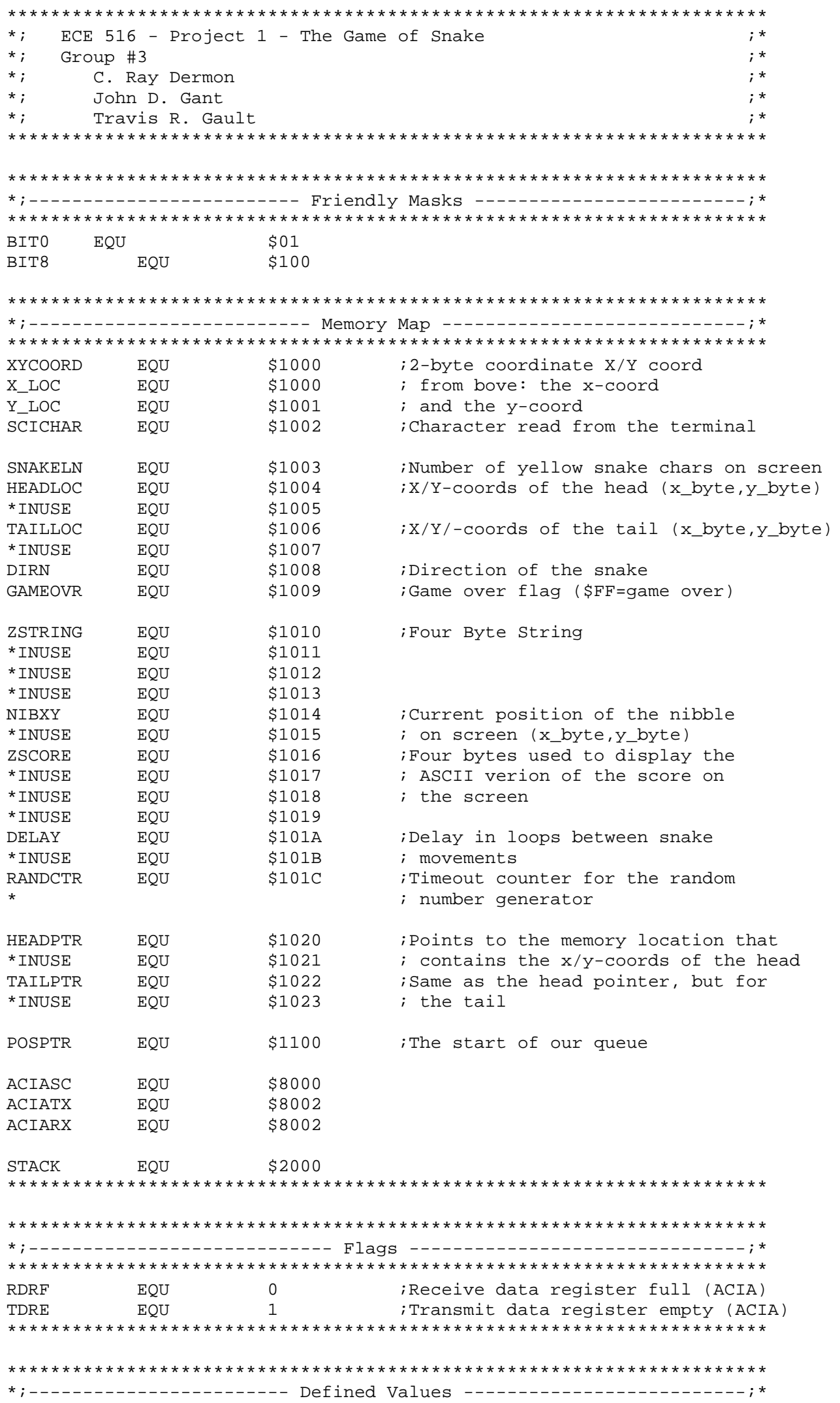




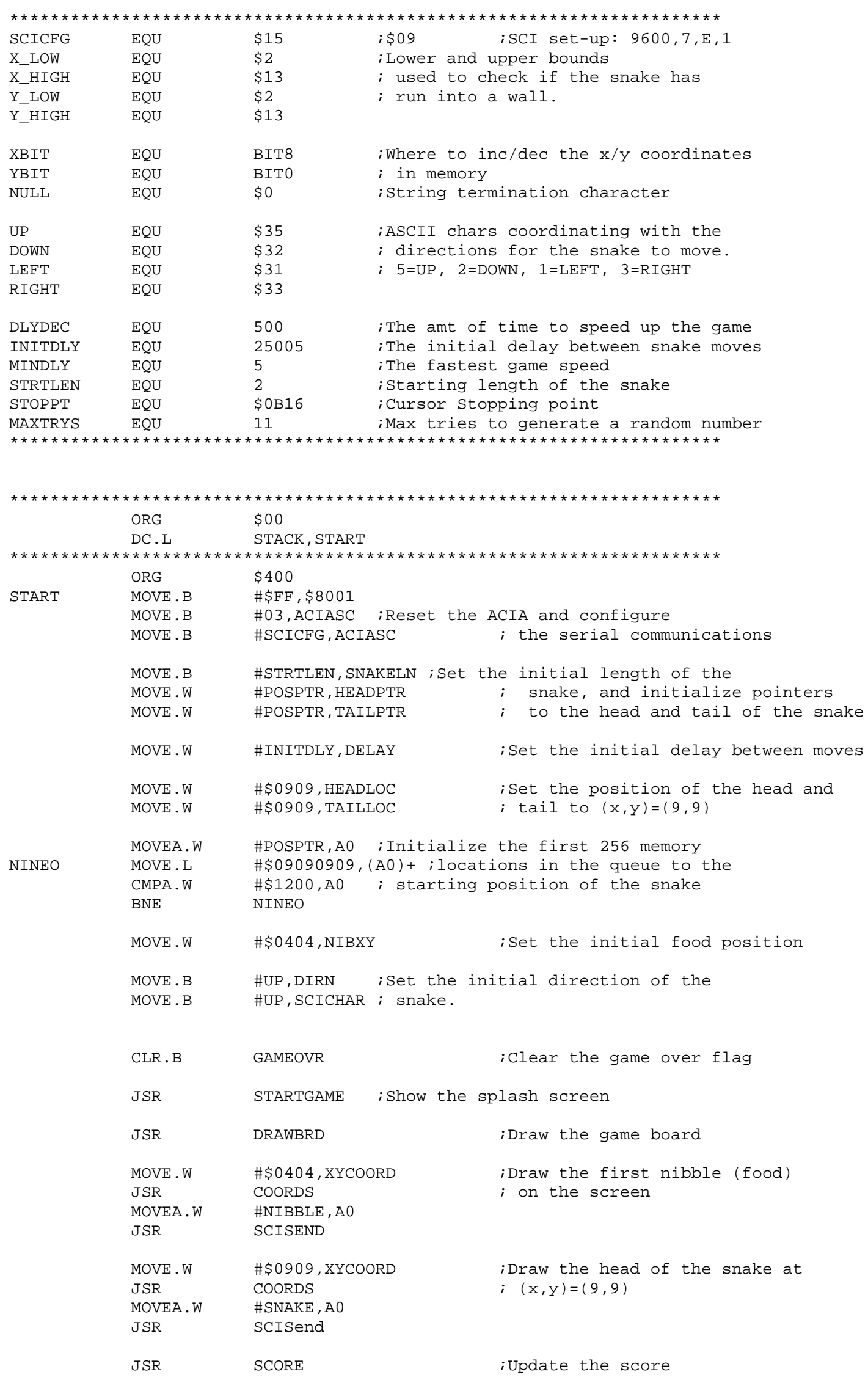




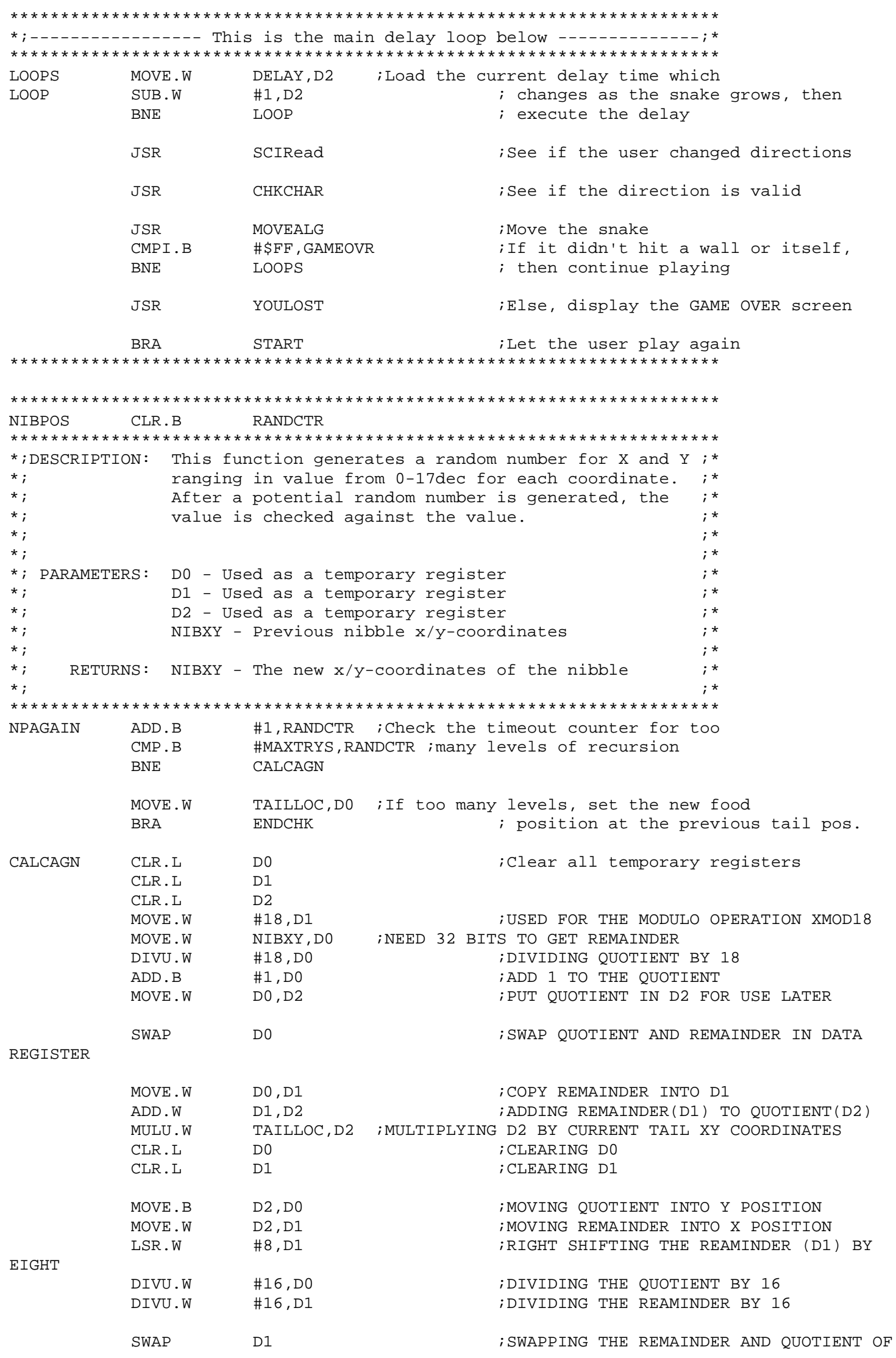


THE PREVIOUS DIVISON

SWAP

THE PREVIOUS DIVISON

$\begin{array}{lll}\text { LSL.W } & \# 8, \text { D1 } & \text {; LEFT SHIFTING D1 AS THE X CORDINATE } \\ \text { ADD.W } & \text { D1, D } & \text {;PUTTING THE X COORDINATE IN D } \\ \text { ADD.W } & \# \$ 202, \text { D } & \end{array}$

$* * * * * * *$ D $\odot$ NOW HAS NEW NIBBLE POSITION $* * * * * * * * * * * *$

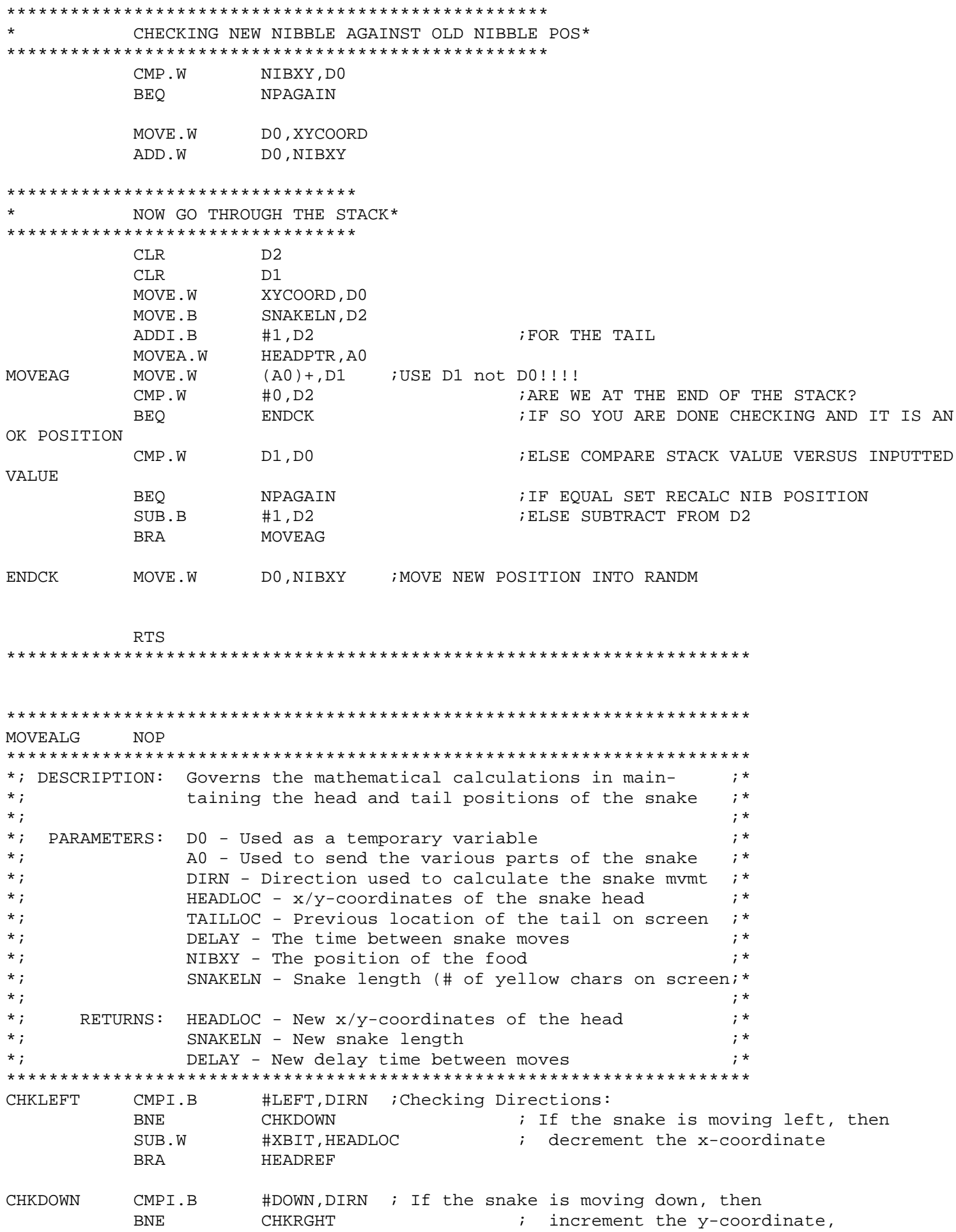




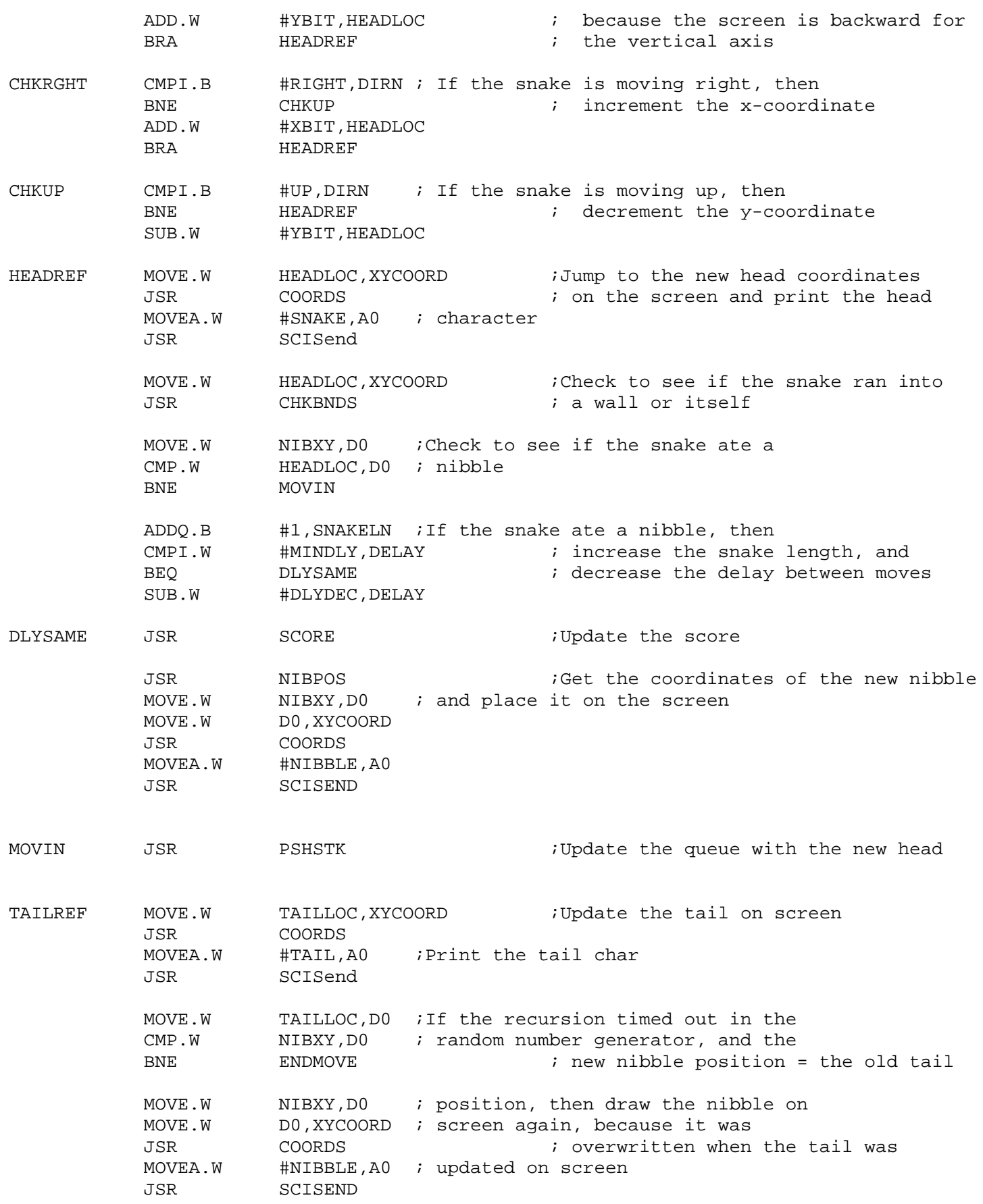

ENDMOVE RTS

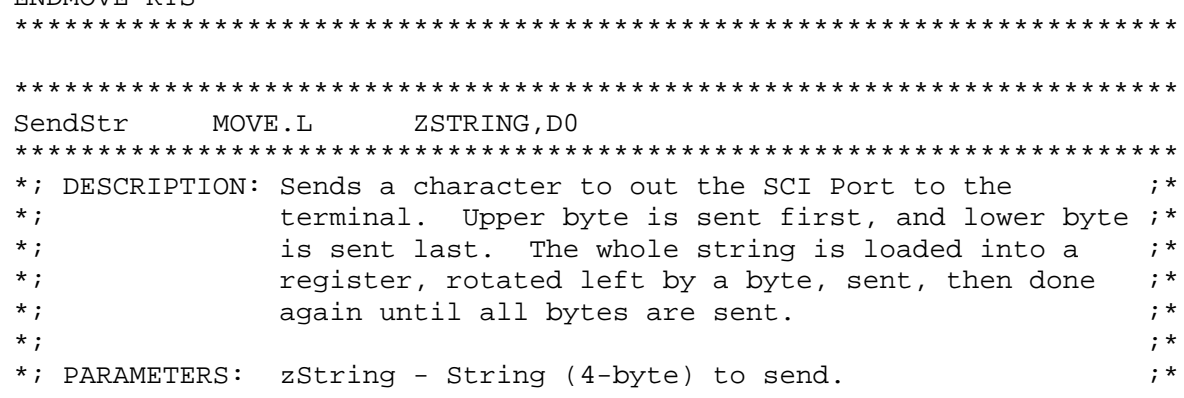




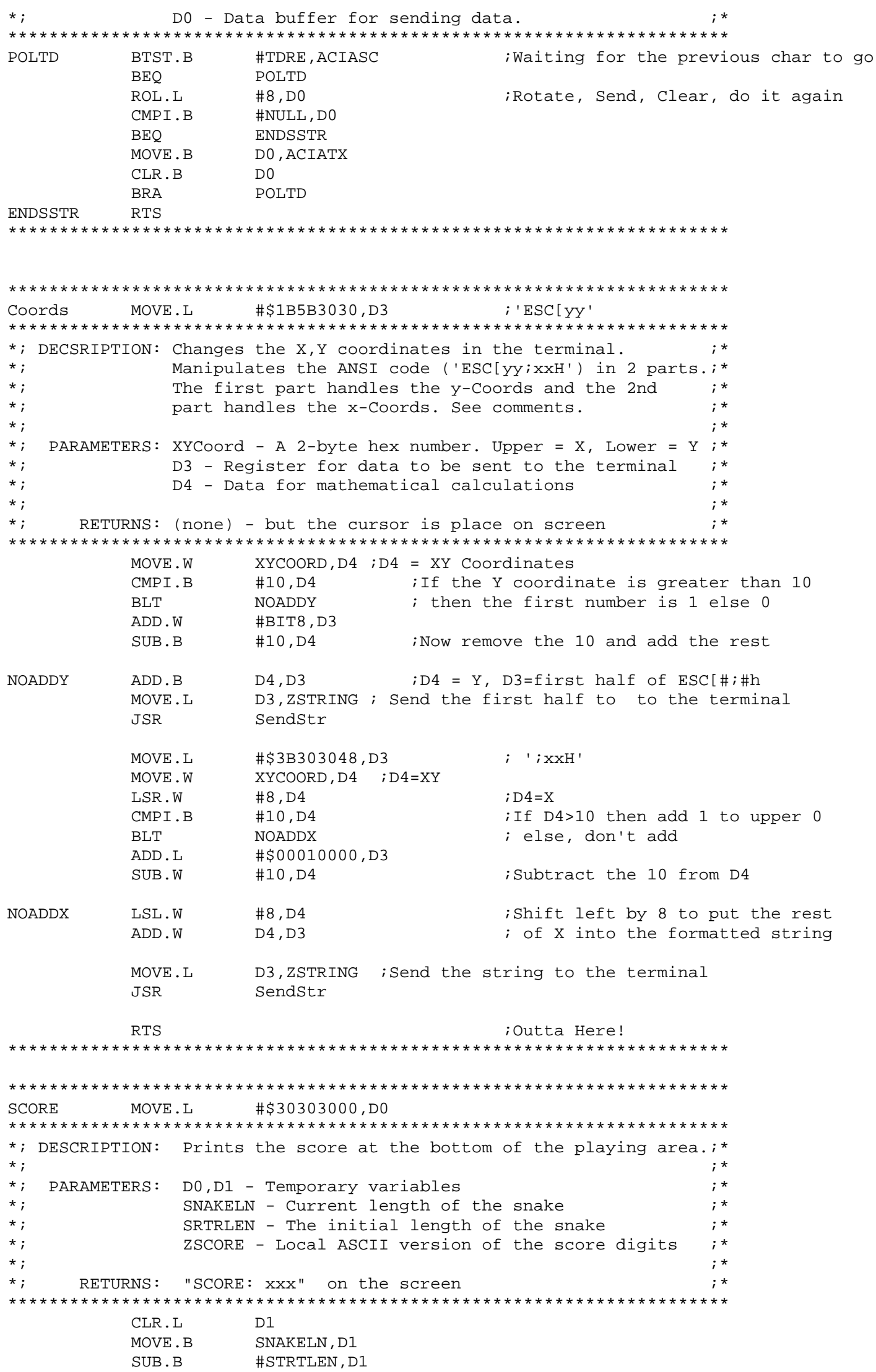




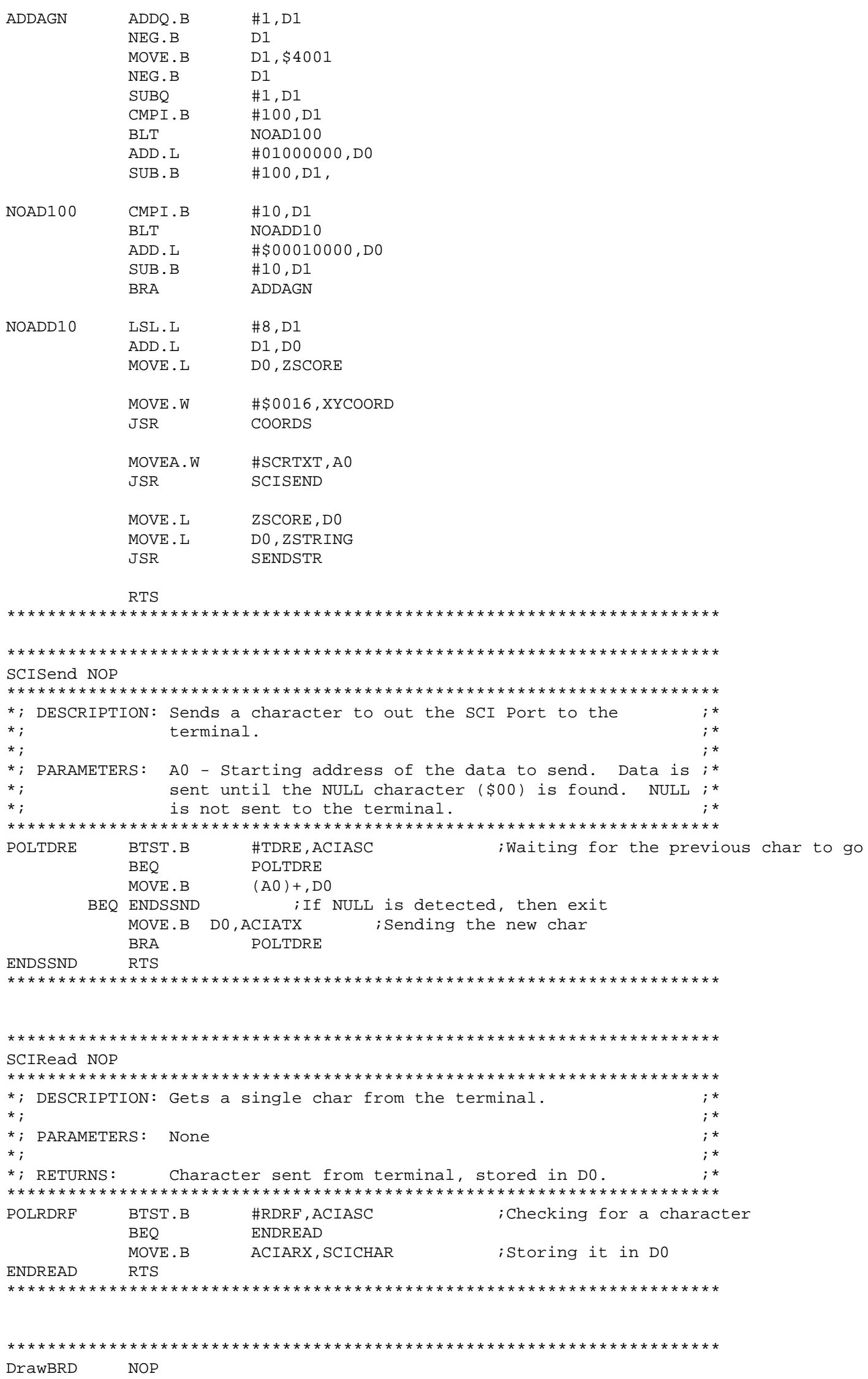




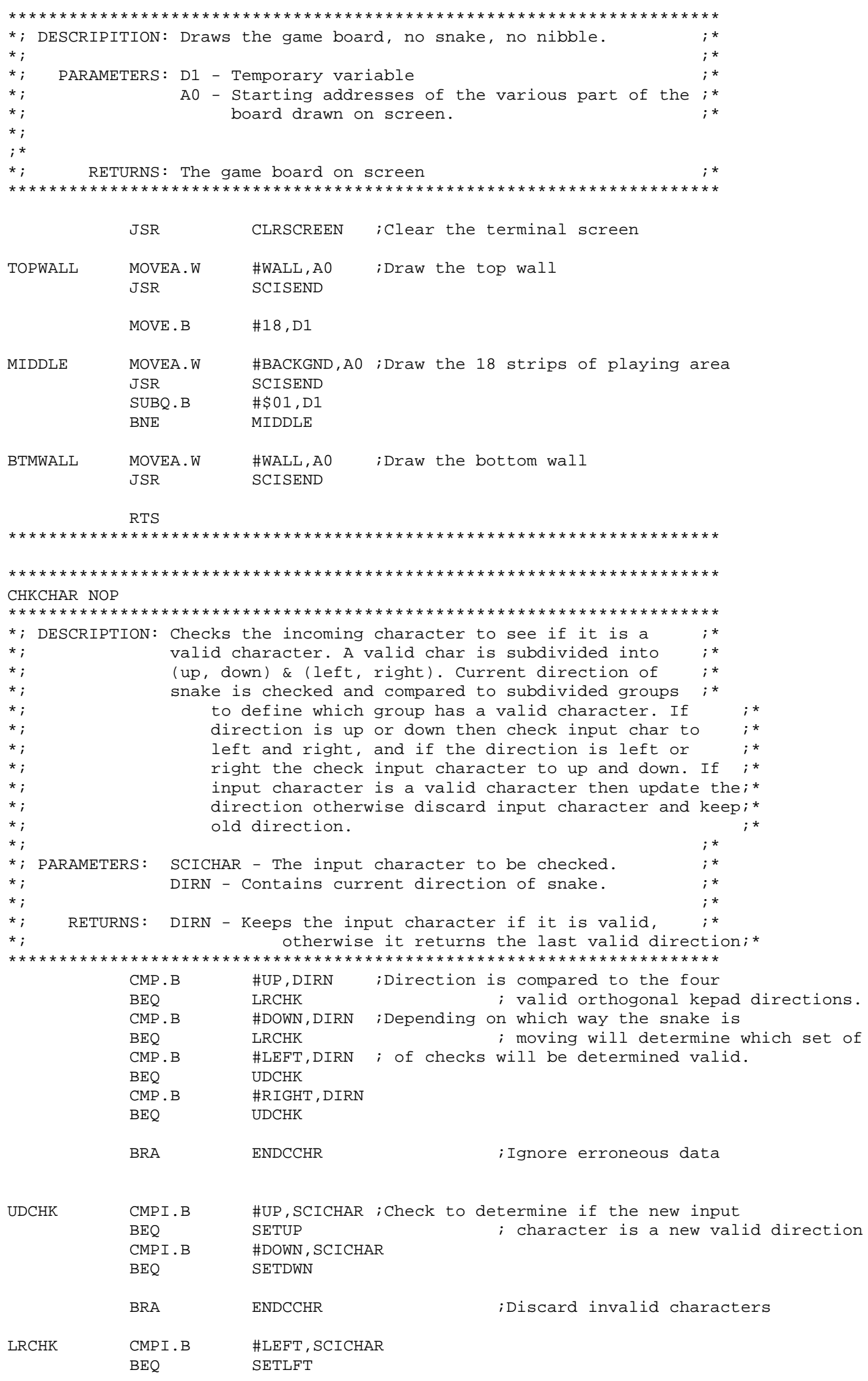




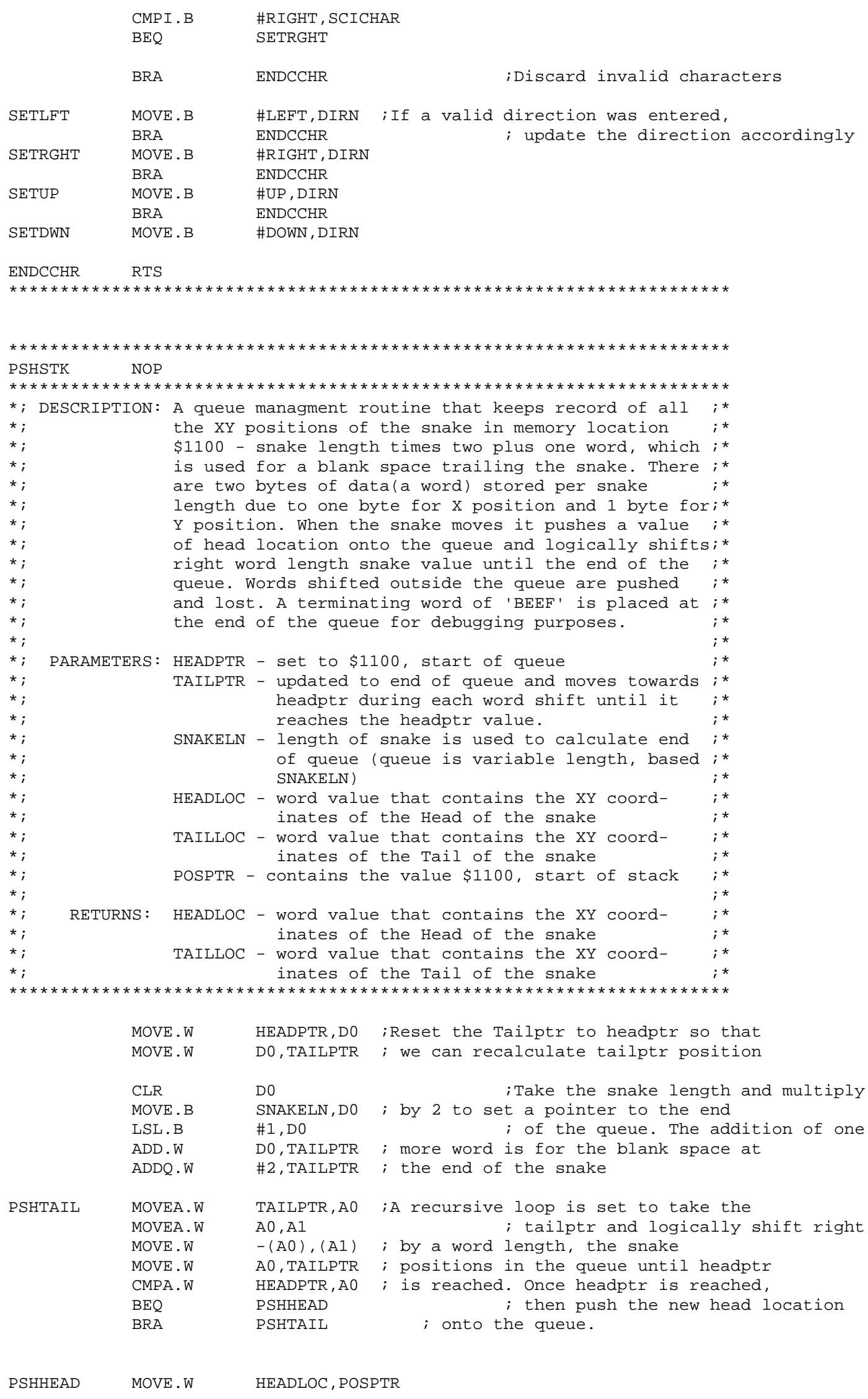




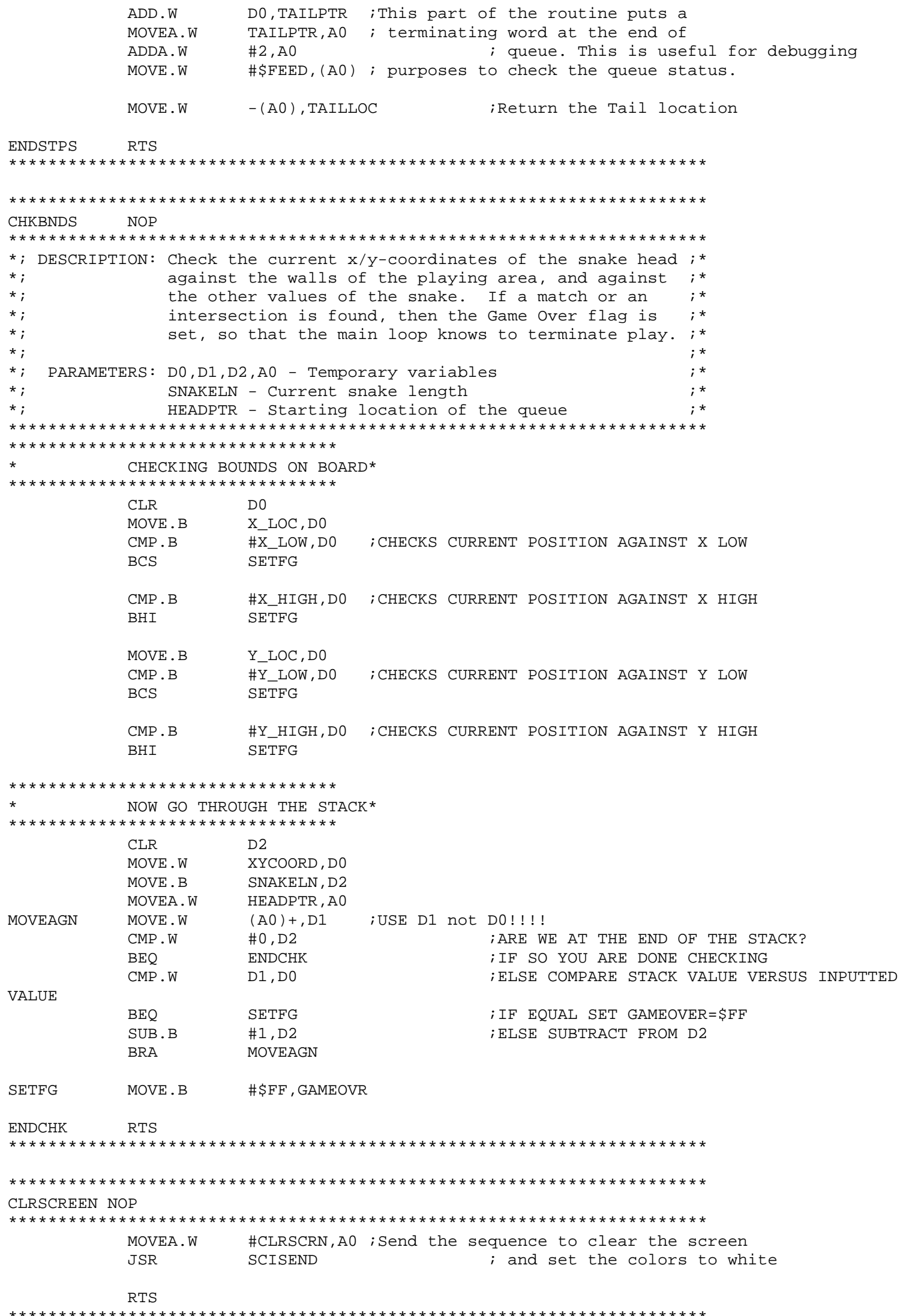




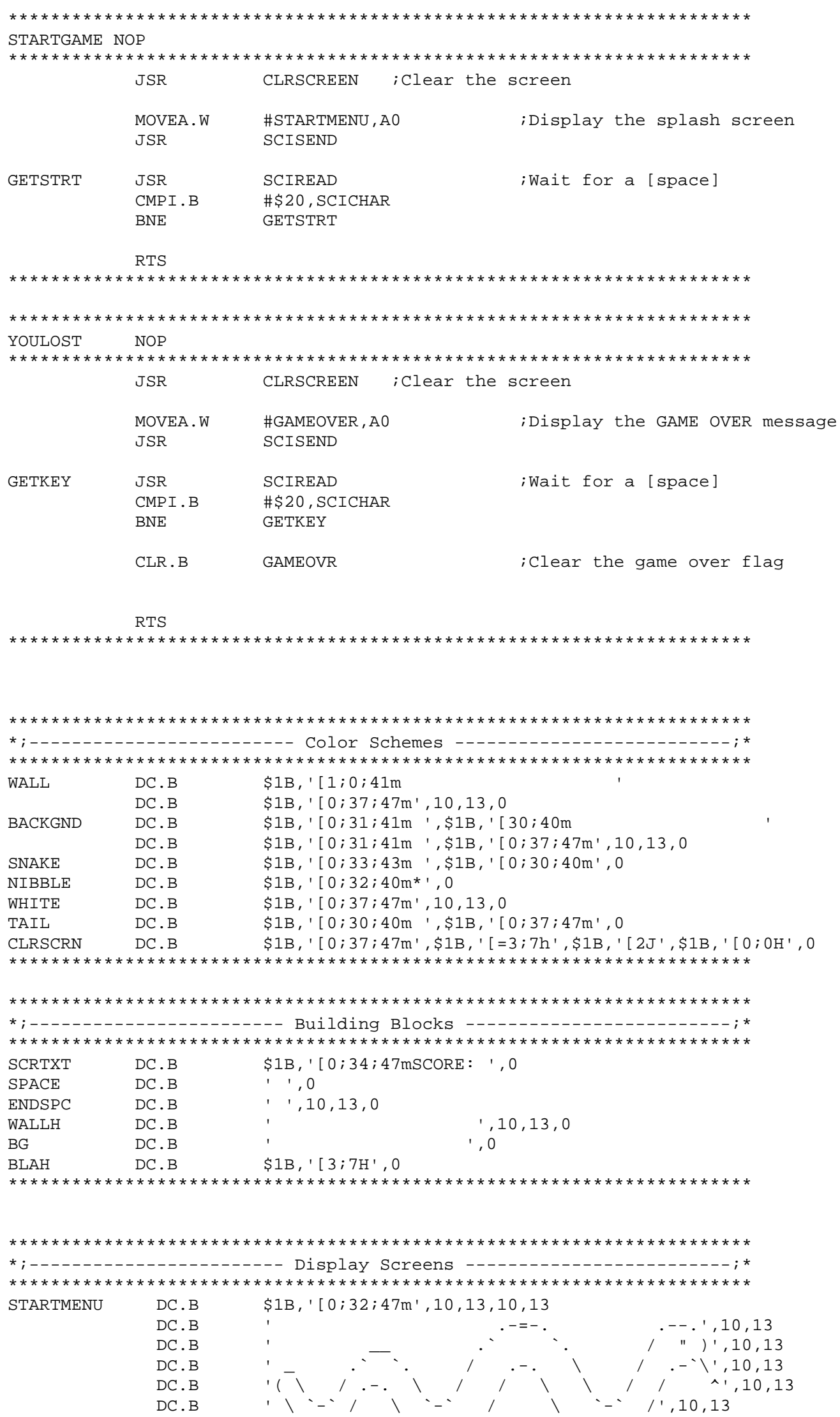




\begin{tabular}{|c|c|}
\hline DC.B & $\therefore ', 10,13,10,13$ \\
\hline DC.B & The Game of Snake', 10,13 \\
\hline$D C . B$ & ' by: Ray Dermon, John Gant, \& Travis Gault', 10, $13,10,13$ \\
\hline DC.B & ' Use the numpad to move: \\
\hline DC.B & $123^{\prime}, 10,13$ \\
\hline DC.B & ' DIRECTIONS: ', 10,13, \\
\hline DC.B & 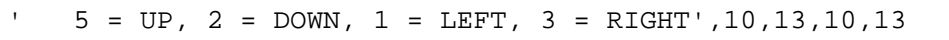 \\
\hline DC.B & ' Don', $\$ 27$, 't Run into walls or yourself.' $, 10,13,10,13$ \\
\hline DC.B & ' Eat the Grub to Grow', $10,13,10,13$ \\
\hline DC.B & 'Press the space key to start. ', 0 \\
\hline
\end{tabular}

GAMEOVER DC.B

$10,13,10,13, \$ 1 B, '\left[0 ; 31 ; 47 \mathrm{~m}^{\prime}\right.$

DC.B ' GGG A M M EEEEE',10,13

DC.B ' G G A A MM MM E',10,13

DC.B 'G A A M M M M EEE',10,13

DC.B IG GG AAAAA M M M E',10,13

DC.B ' $G \quad G \quad A \quad A \quad M \quad M \quad E^{\prime}, 10,13$

DC.B ' GGG A A M M EEEEE',10,13,10,13

DC.B $\quad$ ' $000 \quad \vee \quad V$ EEEEE RRRR', 10,13

DC.B ' 0 O 0 V $\quad V \quad E \quad R \quad R R^{\prime}, 10,13$

DC.B $\quad$ ' 0 O 0 V $V$ EEE $R \quad R^{\prime}, 10,13$

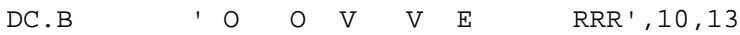

DC.B I $0 \quad 0 \quad \vee V \quad E \quad R \quad R^{\prime}, 10,13$

DC.B ' 000 V EEEEE $R \quad R ', 10,13,10,13$

DC. B \$1B, '[0;30;47mpress the space key to start over. ', $\odot$

END $\$ 200 \odot$ 


\section{APPENDIX IV - DESIGN SOURCE CODE}

\begin{tabular}{|c|c|c|c|}
\hline AVIRQ1 & EQU & $\$ 64$ & ;Auto-Vector Interrupt Level 1 \\
\hline FO & EQU & $\$ 1000$ & ;FO.W contains the fundamental frequency \\
\hline *;IN USE & EQU & $\$ 1001$ & \\
\hline FDEC & EQU & $\$ 1002$ & \\
\hline *;IN USE & EQU & $\$ 1003$ & \\
\hline IRQENFLAG & EQU & $\$ 1004$ & ;Current status of interrupts \\
\hline MIDIFLAGS & EQU & $\$ 1005$ & ;MIDI flags for sending notes \\
\hline MIDIINST & EQU & $\$ 1006$ & ; Location containing Instrument $\mathrm{Nbr}$. \\
\hline ERRCOUNT & EQU & $\$ 1007$ & ; Number of times there are $f \odot$ errors \\
\hline KNVAL & EQU & \$14E0 & ;Current value of $\mathrm{k}+\mathrm{n}$ \\
\hline *; IN USE & EQU & $\$ 1001$ & \\
\hline $\mathrm{x}$ & EQU & $\$ 14 \mathrm{~F} 0$ & ;Temporary summation variable \\
\hline *;IN USE & EQU & $\$ 1001$ & \\
\hline NMAX & EQU & $\$ 14 \mathrm{~F} 4$ & ;Maximum allowable value of $\mathrm{N}$ \\
\hline *;IN USE & EQU & $\$ 1005$ & \\
\hline KMAX & EQU & \$14F6 & ;Maximum allowable value of $\mathrm{K}$ \\
\hline *;IN USE & EQU & $\$ 1007$ & \\
\hline KNMAX & EQU & $\$ 14 F 8$ & ; Maximum allowable value of $\mathrm{K}+\mathrm{N}$ \\
\hline *;IN USE & EQU & $\$ 1009$ & \\
\hline DDMAX & EQU & \$14FA & ;Maximum array index for AMDF results \\
\hline *;IN USE & EQU & $\$ 100 \mathrm{~B}$ & \\
\hline MAXOFDD & EQU & \$14FC & ;Max\{AMDF_RESULTS $\}$ \\
\hline *; IN USE & EQU & $\$ 100 \mathrm{D}$ & \\
\hline FMAX & EQU & \$14FE & ;Maximum array index for FINDEX \\
\hline *; IN USE & EQU & $\$ 100 \mathrm{~F}$ & \\
\hline STATUS & EQU & \$10FA & ;MIDI Command \\
\hline NOTENBR & EQU & \$10FB & ;MIDI Note number \\
\hline PROGNUM & EQU & \$10FB & ;MIDI Program number \\
\hline NOTEVEL & EQU & \$10FC & ;MIDI Note Velocity \\
\hline MSGEND & EQU & \$100D & ; End of Message \\
\hline PREVMIDI & EQU & \$10FE & ;Previous MIDI note sent \\
\hline MIDINOTE & EQU & \$10FF & ;Current MIDI note to send \\
\hline $\mathrm{S}$ & EQU & $\$ 1100$ & ; Sampled data from ADC \\
\hline DD & EQU & $\$ 1300$ & ;ADMF results (word lengths) \\
\hline FINDEX & EQU & $\$ 1700$ & ;Array indices for dips in AMDF \\
\hline ENDRAM & EQU & $\$ 4000$ & ; End of RAM memory location +1 \\
\hline LATCH & EQU & $\$ 4001$ & ; Location of register for LEDS \\
\hline ADCO & EQU & $\$ 4000$ & ;A/D converter channel \\
\hline MIDISC & EQU & $\$ 8001$ & ;MIDI ACIA status/control register \\
\hline MIDITXD & EQU & $\$ 8003$ & ;MIDI ACIA transmit data register \\
\hline MIDIRXD & EQU & $\$ 8003$ & ;MIDI ACIA receive data register \\
\hline SCISC & EQU & $\$ 8000$ & ;MIDI ACIA status/control register \\
\hline SCITXD & EQU & $\$ 8002$ & ;MIDI ACIA transmit data register \\
\hline SCIRXD & EQU & $\$ 8002$ & ;MIDI ACIA receive data register \\
\hline
\end{tabular}




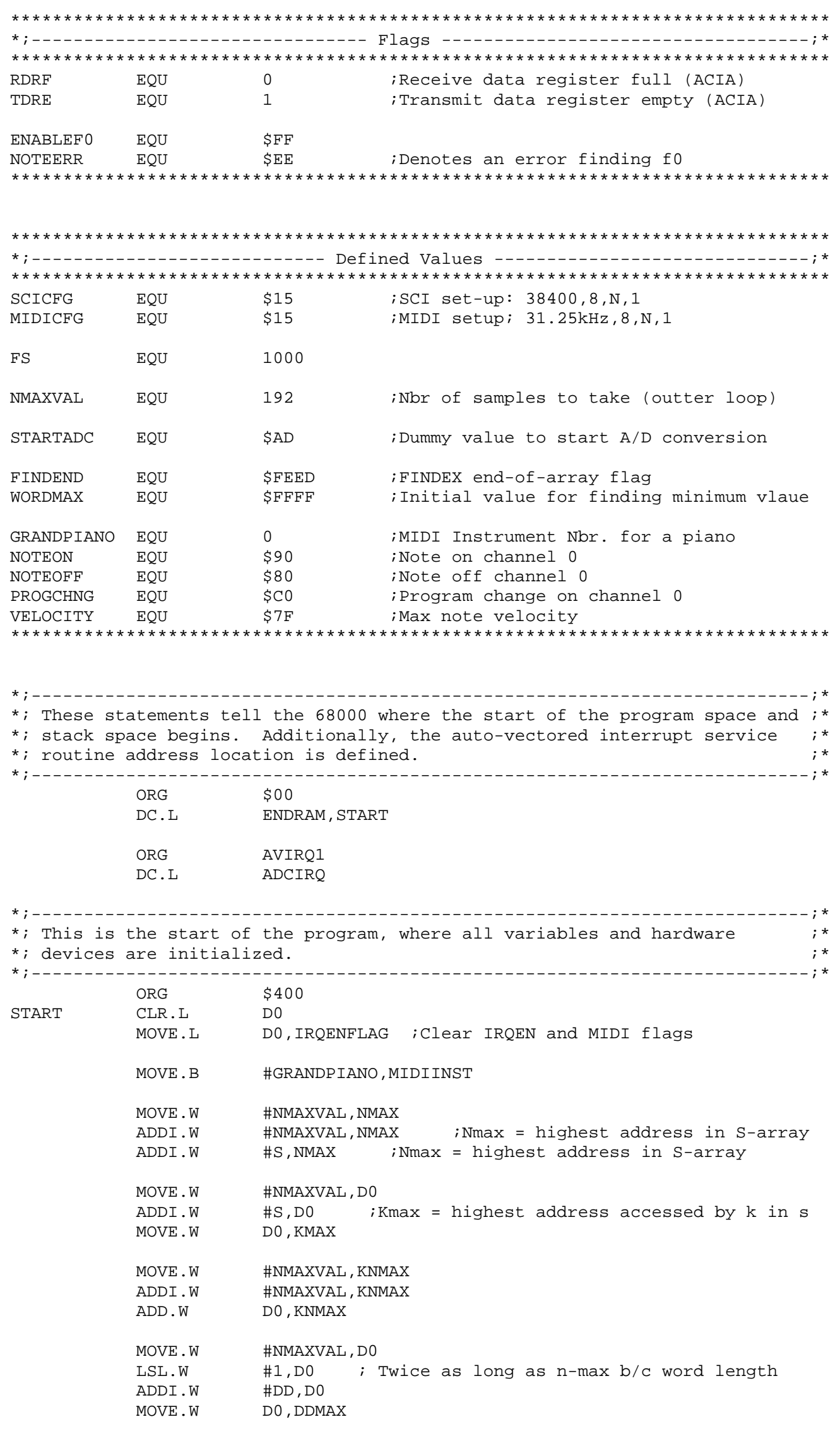




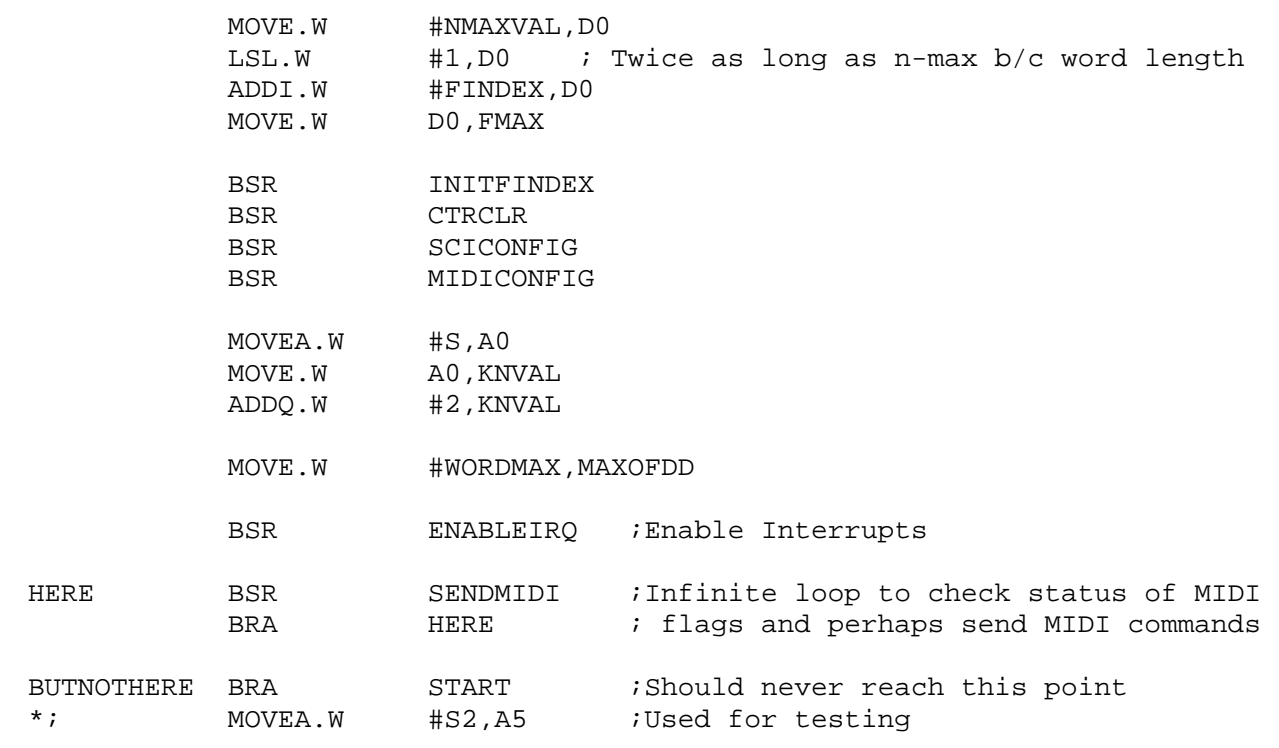

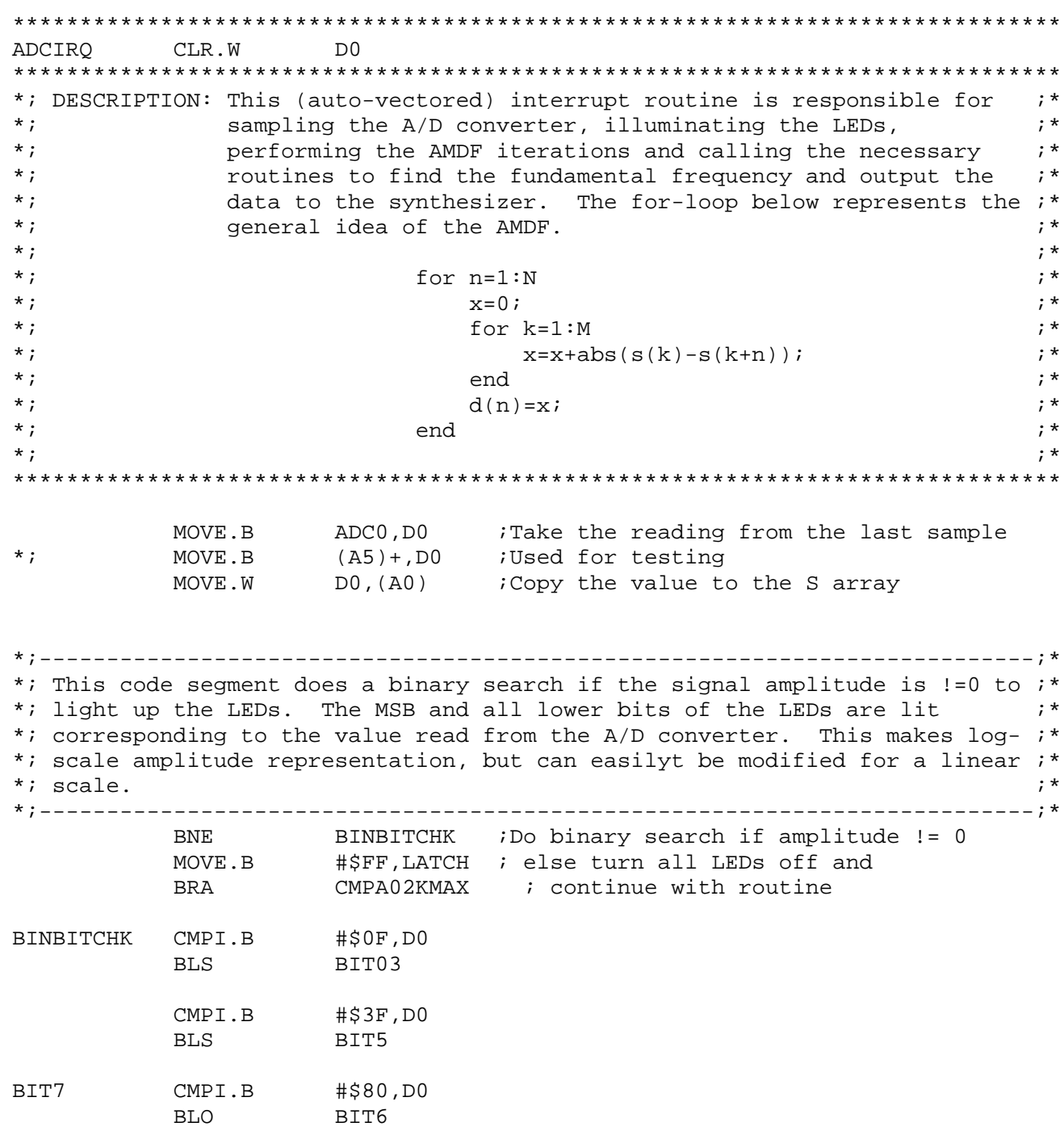




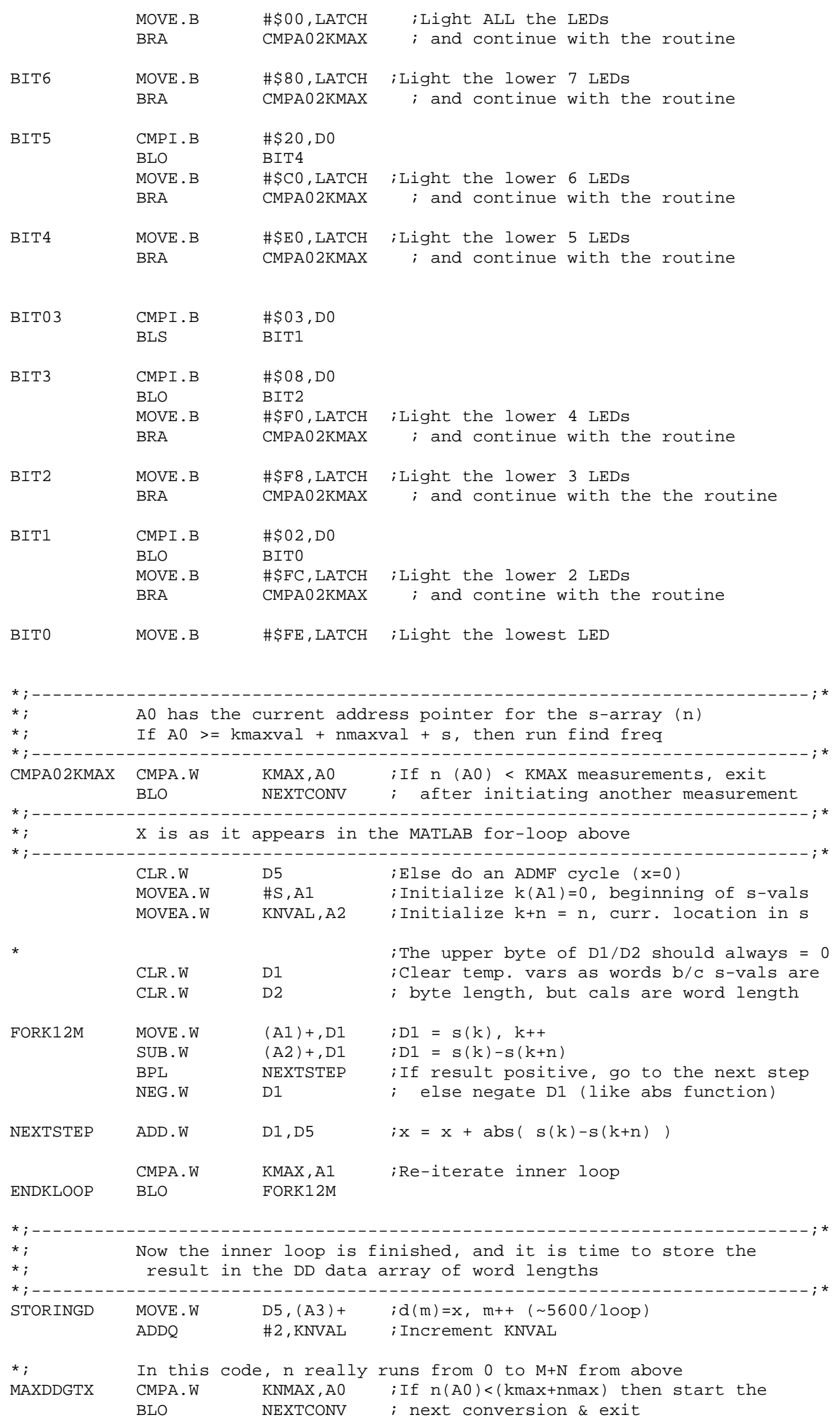




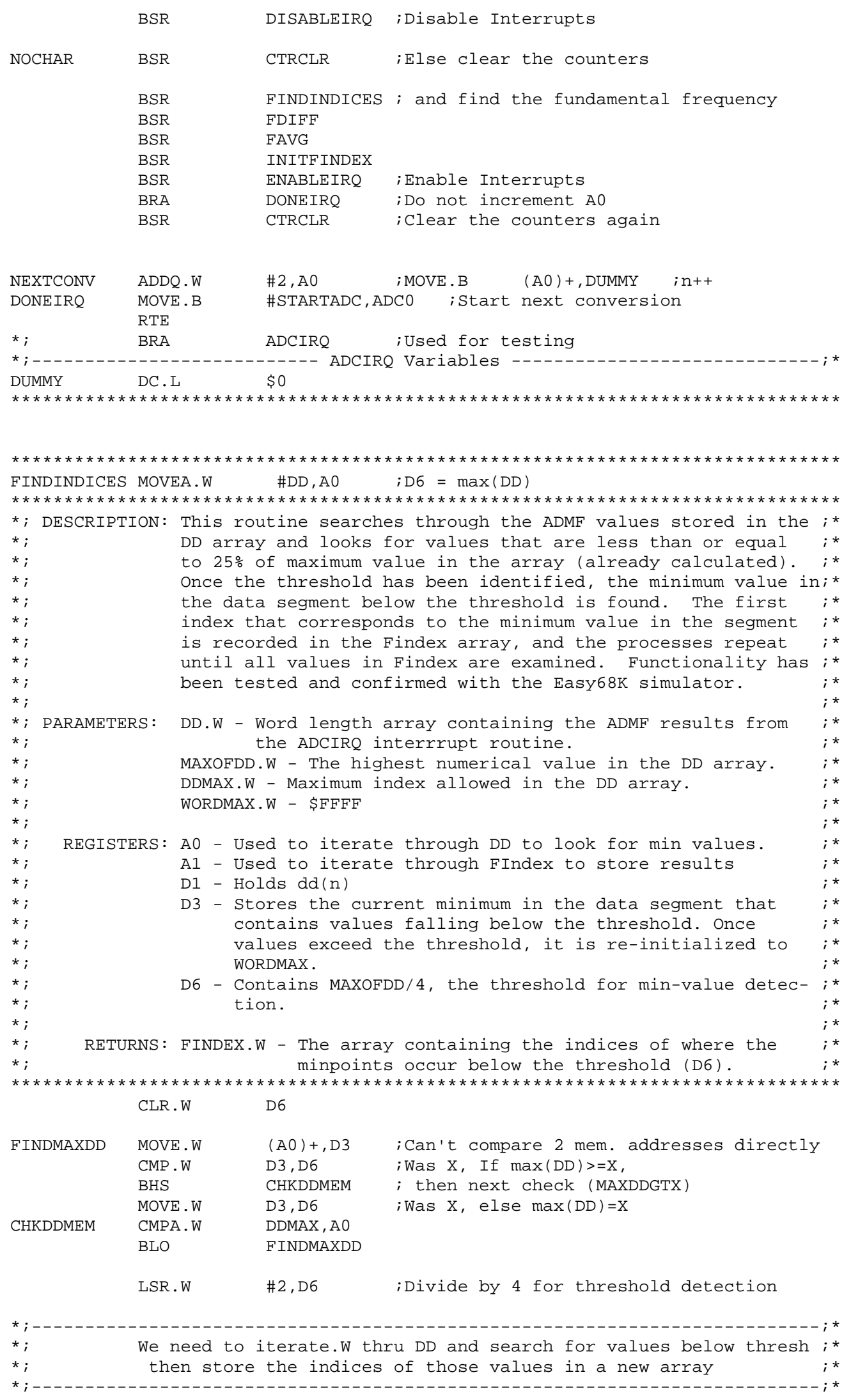




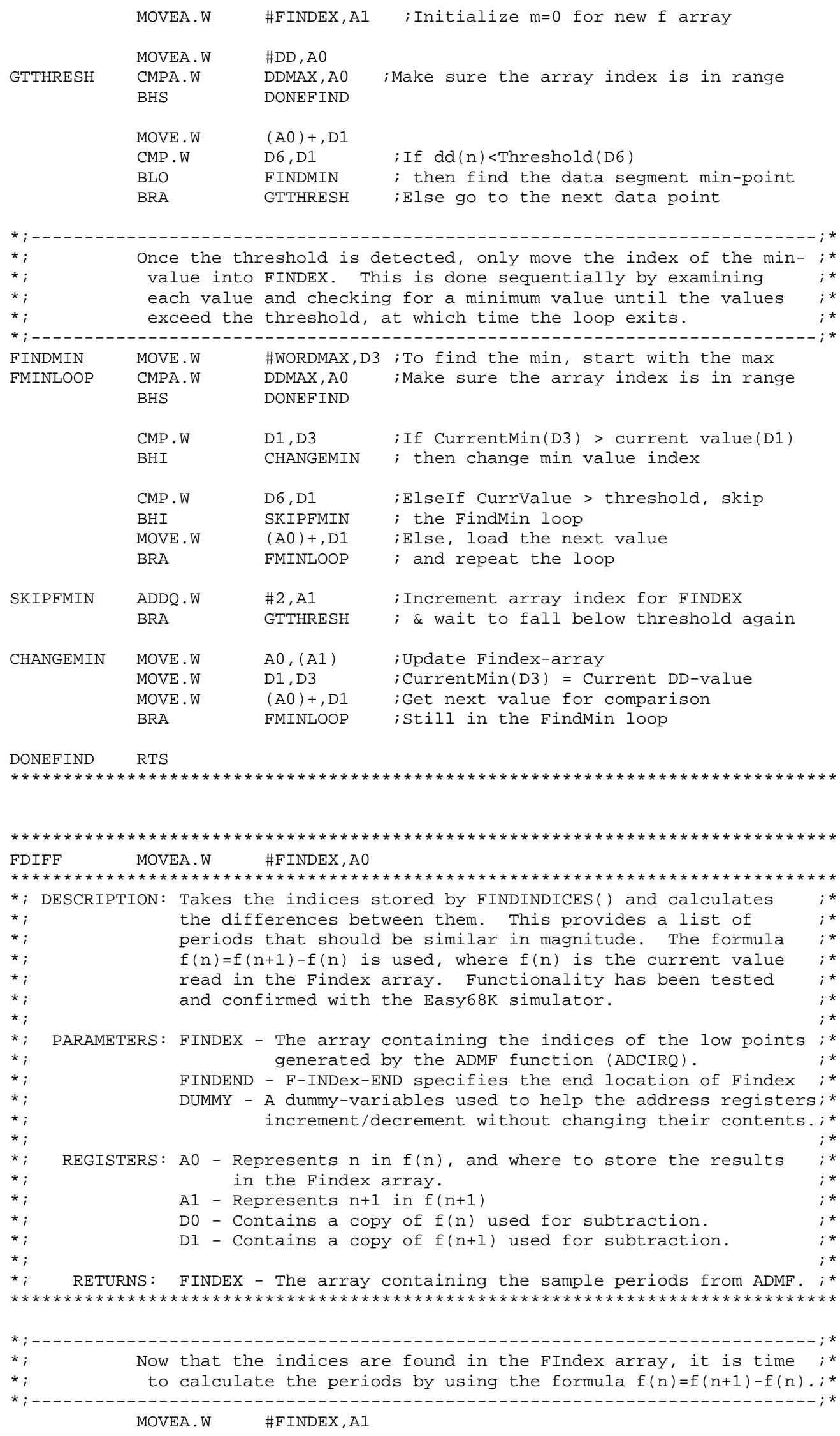




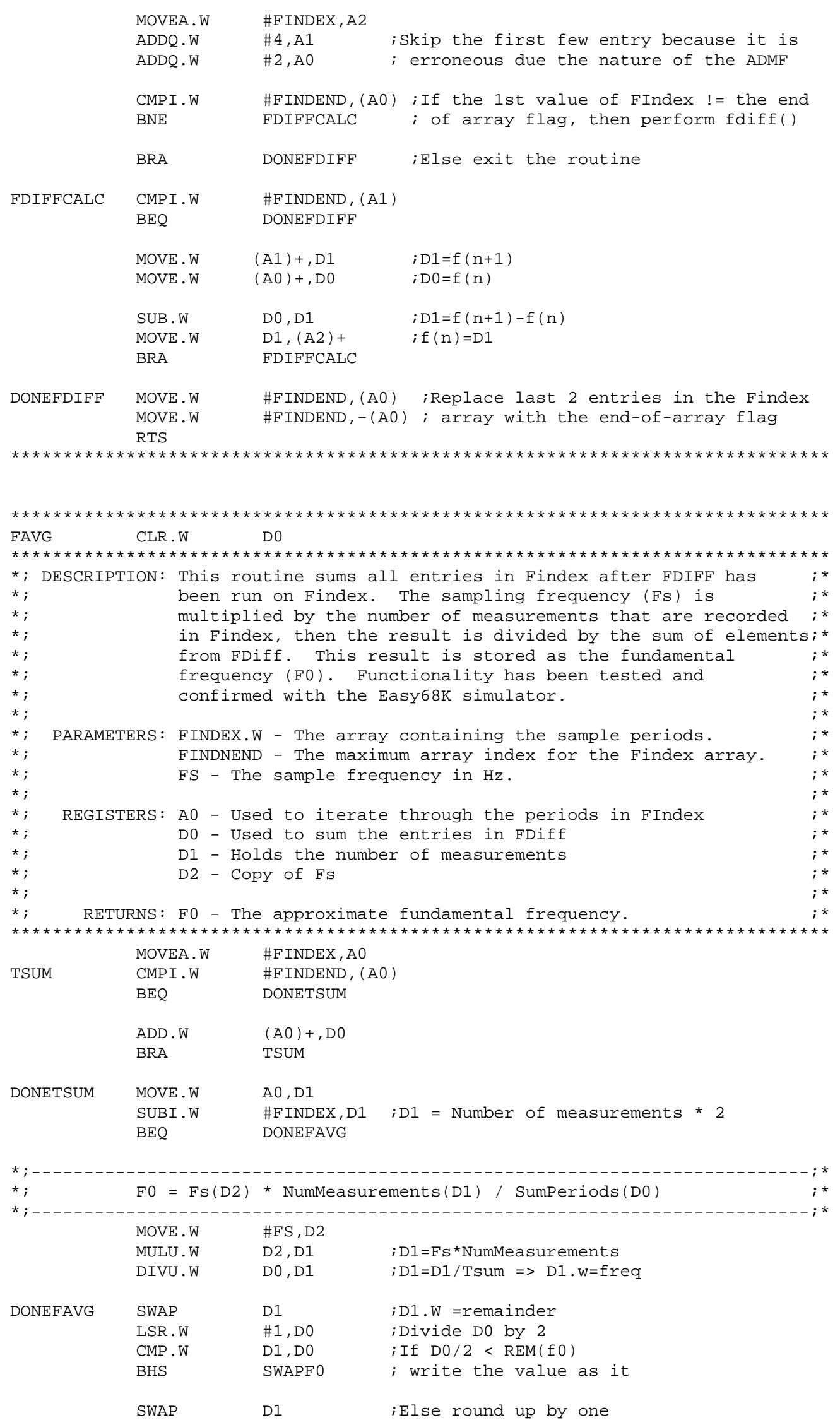




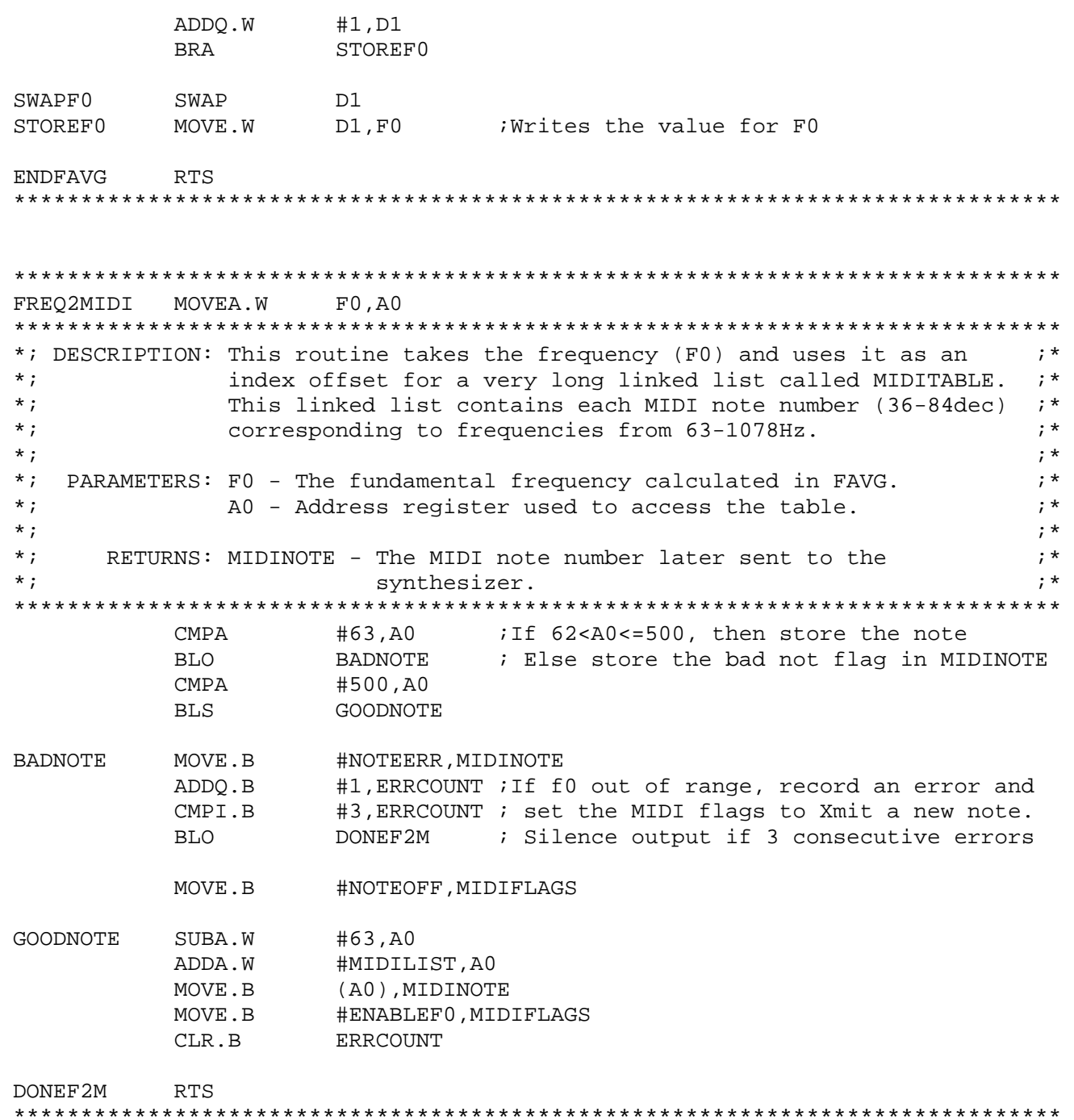

\begin{tabular}{|c|c|}
\hline $\begin{array}{l}\text { SENDMIDI MOVE } \\
* * * * * * * * * * * * * * * *\end{array}$ & 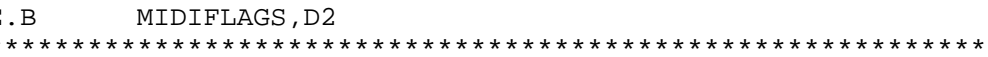 \\
\hline DESCRIPTION : & $\begin{array}{l}\text { This routine examines the MIDI flags set in this software } \\
\text { and takes action based on these flags. If the flags are } \\
\text { clear, then the routine exits. If the NOTE OFF flag is } \\
\text { detected, then the previous MIDI note is disabled. If any } \\
\text { other value is detected, then a MIDI packet instructing the } \\
\text { synthesizer to change notes is sent if the current note } \\
\text { differs from the previous note. }\end{array}$ \\
\hline PARAMETERS: & $\begin{array}{l}\text { MIDIFLAGS - Determines what action to take. } \\
\text { PREVMIDI - Previous MIDI note sent } \\
\text { MIDINOTE - Current MIDI note to evaluate }\end{array}$ \\
\hline REGISTERS: & $\begin{array}{l}\text { DO - Contains the previous MIDI note } \\
\text { D1 - Contains the current MIDI note } \\
\text { D2 - Contains the MIDI flags }\end{array}$ \\
\hline
\end{tabular}

BEQ DONESNDMIDI; if the flags are clear, then exit

CMPI.B \#NOTEOFF,D2 ; Are we to disable the previous note w/o BNE NEWNOTE ; sending another note? If not, send note 


\begin{tabular}{|c|c|c|}
\hline \multirow[t]{2}{*}{ SILENCE } & $\begin{array}{l}\text { MOVE. B } \\
\text { MOVE. B } \\
\text { MOVE. B } \\
\text { MOVE. B } \\
\text { MOVE. W } \\
\text { BSR }\end{array}$ & $\begin{array}{l}\text { \#NOTEOFF, STATUS ;Else, silence the previous note } \\
\text { PREVMIDI, NOTENBR } \\
\text { \#VELOCITY, NOTEVEL } \\
\text { \#O, MSGEND } \\
\text { \#STATUS, MIDISTR } \\
\text { MIDISend }\end{array}$ \\
\hline & BRA & DONESNDMIDI \\
\hline \multirow[t]{4}{*}{ NEWNOTE } & $\begin{array}{l}\text { MOVE. B } \\
\text { MOVE. B } \\
\text { CMP. B } \\
\text { BEQ }\end{array}$ & $\begin{array}{l}\text { PREVMIDI, D० } \\
\text { MIDINOTE, D1 ; If the previous note and current note } \\
\text { D0,D1 } \\
\text { DONESNDMIDI } ; \text { are the same, there is no need to send } \\
\text { other MIDI packets, so exit }\end{array}$ \\
\hline & $\begin{array}{l}\text { CMPI . B } \\
\text { BEQ }\end{array}$ & $\begin{array}{l}\text { \#NOTEERR,D1 ;Exit if an error identifying fo } \\
\text { DONESNDMIDI }\end{array}$ \\
\hline & $\begin{array}{l}\text { MOVE. B } \\
\text { MOVE. B } \\
\text { MOVE. B } \\
\text { MOVE. B } \\
\text { MOVE. W } \\
\text { BSR }\end{array}$ & $\begin{array}{l}\text { \#NOTEOFF, STATUS ;Else, silence the previous note } \\
\text { PREVMIDI, NOTENBR } \\
\text { \#VELOCITY, NOTEVEL } \\
\text { \#O, MSGEND } \\
\text { \#STATUS, MIDISTR } \\
\text { MIDISend }\end{array}$ \\
\hline & $\begin{array}{l}\text { MOVE. B } \\
\text { MOVE. B } \\
\text { BSR }\end{array}$ & $\begin{array}{l}\text { \#NOTEON, STATUS ;And send the new note and exit } \\
\text { MIDINOTE, NOTENBR } \\
\text { MIDISend }\end{array}$ \\
\hline DONESNDMIDI & CLR.B & MIDIFLAGS \\
\hline
\end{tabular}

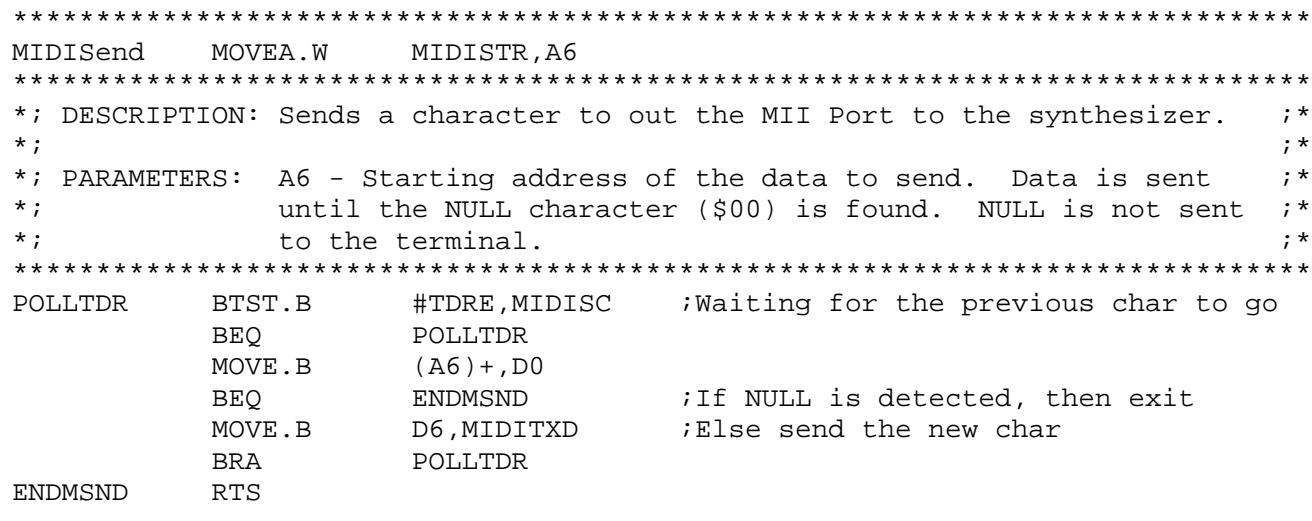

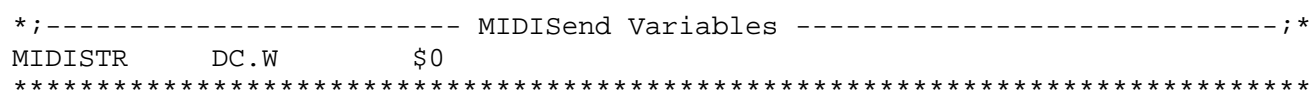

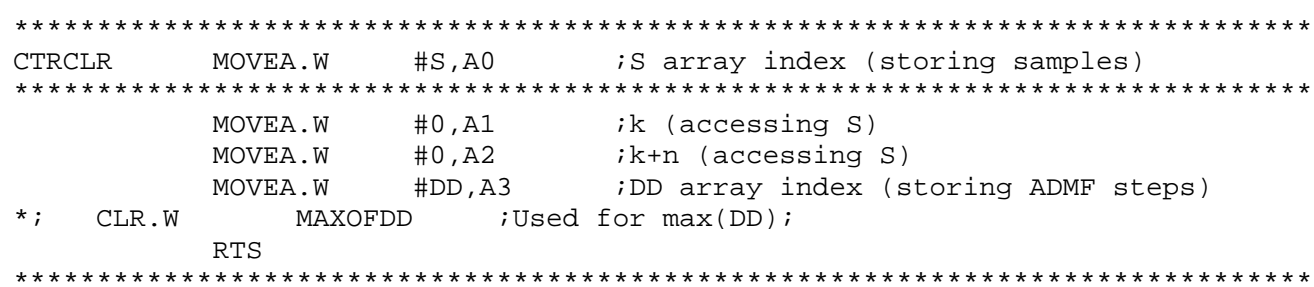




\begin{tabular}{|c|c|c|}
\hline$\star \star \star * * * \star * * *$ & $\begin{array}{l}\text { MOVE.W } \\
\text { MOVE.W } \\
\text { CMPA.W } \\
\text { BLS } \\
\text { RTS } \\
* * * * * * *\end{array}$ & 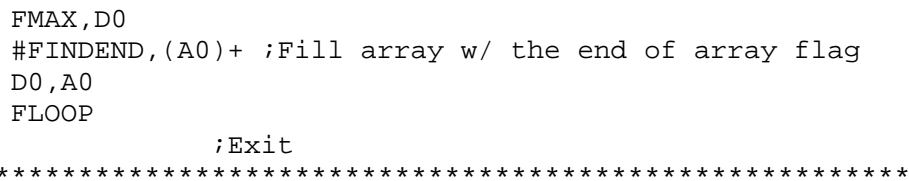 \\
\hline \multicolumn{3}{|c|}{ 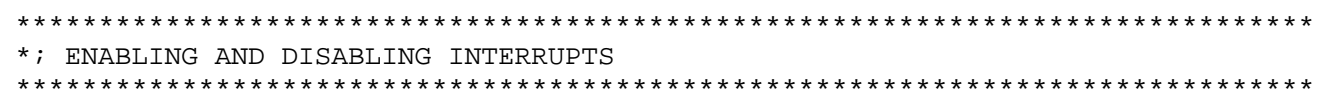 } \\
\hline ENABLEIRQ & $\begin{array}{l}\text { ANDI.W } \\
\text { MOVE. B } \\
\text { RTS }\end{array}$ & $\begin{array}{l}\text { \#\$F8FF, SR } \\
\# \$ F F, I R Q E N F L A G\end{array}$ \\
\hline DISABLEIRQ & $\begin{array}{l}\text { ORI.W } \\
\text { MOVE.B } \\
\text { RTS } \\
\star \star \star \star \star \star \star * \star\end{array}$ & $\begin{array}{l}\# \$ \odot 7 \odot \odot, \text { SR } \\
\# \$ \odot \odot, \text { IRQENFLAG }\end{array}$ \\
\hline \multicolumn{3}{|c|}{ 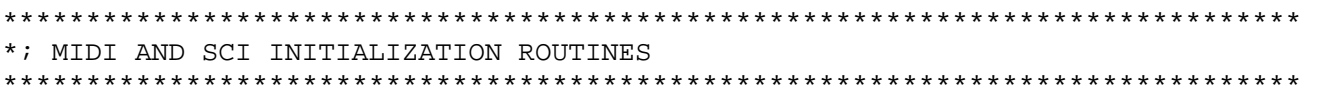 } \\
\hline SCICONFIG & $\begin{array}{l}\text { MOVE. B } \\
\text { MOVE. B } \\
\text { RTS }\end{array}$ & $\begin{array}{l}\# \$ 3, \text { SCISC } \\
\# \text { SCICFG, SCISC }\end{array}$ \\
\hline MIDICONFIG & $\begin{array}{l}\text { MOVE.B } \\
\text { MOVE. B } \\
\text { RTS }\end{array}$ & $\begin{array}{l}\text { \#\$3, MIDISC } \\
\text { \#MIDICFG, MIDISC }\end{array}$ \\
\hline & INCLUDE & 'MIDIList.x68' \\
\hline & END & STA \\
\hline
\end{tabular}


MIDIList . $\times 68$

\begin{tabular}{|c|c|c|c|c|c|}
\hline RG & $\begin{array}{l}2000 \\
* * * * *\end{array}$ & & & & \\
\hline Pitch I & $\mathrm{F} \odot$ & I Valid Fos & Offsets & MIDI & ; \\
\hline C2 & 65.406 & $63-67$ & $0-4$ & 36 & \\
\hline $\mathrm{C \# 2}$ & 69.296 & $68-71$ & $5-8$ & 37 & \\
\hline D2 & 73.416 & $72-75$ & $9-12$ & 38 & \\
\hline D\#2 & 77.782 & $76-80$ & $13-17$ & 39 & \\
\hline E2 & 82.407 & $81-84$ & $18-21$ & 40 & ; * \\
\hline $\mathrm{F} 2$ & 87.307 & $85-89$ & $22-26$ & 41 & * \\
\hline $\mathrm{F} \# 2$ & 92.499 & $90-95$ & $27-32$ & 42 & ; * \\
\hline G2 & 97.999 & $96-100$ & $33-37$ & 43 & $;^{*}$ \\
\hline $\mathrm{G} \# 2$ & 103.826 & $101-106$ & $38-43$ & 44 & \\
\hline A2 & 110 & $107-113$ & $44-50$ & 45 & ; * \\
\hline A\#2 & 116.541 & $114-120$ & $51-57$ & 46 & ; * \\
\hline B2 & 123.471 & $121-127$ & $58-64$ & 47 & ; * \\
\hline C3 & 130.813 & $128-134$ & $65-71$ & 48 & $;^{*}$ \\
\hline C\#3 & 138.591 & $135-1$ & $72-79$ & 49 & 1 \\
\hline D3 & 146 & $143-1$ & 80 & 50 & $i^{*}$ \\
\hline D\#3 & 15 & $152-1$ & & 51 & ; * \\
\hline E3 & 164.814 & $161-1$ & 98 & 52 & ; * \\
\hline F3 & 174.614 & $170-179$ & $107-$ & 53 & ; * \\
\hline F\#3 & 184.997 & $180-1$ & $117-$ & 54 & ; * \\
\hline G3 & 19 & $1-2$ & & 55 & ; * \\
\hline $\mathrm{G} \# 3$ & 207 & 2 & & 56 & ; * \\
\hline A3 & 220 & $14-226$ & $151-$ & 57 & ; * \\
\hline A\#3 & 233.0 & $27-240$ & $164-177$ & 58 & ; \\
\hline B3 & 246 & $41-2$ & 17 & 59 & ; * \\
\hline $\mathrm{C} 4$ & & -2 & $1 \mathrm{~s}$ & 60 & ; * \\
\hline C\#4 & 277 & $270-2$ & 20 & 61 & ; * \\
\hline D4 & 293 & $6-3$ & 22 & 62 & ; * \\
\hline D\#4 & 311.127 & $03-320$ & $240-257$ & 63 & ; * \\
\hline E4 & 329.628 & $321-3$ & $258-276$ & 64 & ; * \\
\hline F4 & 34 & $1-3$ & 2 & 65 & ; * \\
\hline $\mathrm{F} \# 4$ & $36 s$ & 3 & 29 & 66 & $;^{*}$ \\
\hline G4 & 391.9 & $1-4 \odot 3$ & 31 & 67 & ; * \\
\hline G\#4 & 415.305 & $404-427$ & $341-364$ & 68 & $i^{*}$ \\
\hline A4 & 440 & $8-453$ & $365-390$ & 69 & $i^{\prime}$ \\
\hline A\#4 & & 4 & & 70 & $\therefore *$ \\
\hline B4 & & 4 & 41 & 71 & $\therefore *$ \\
\hline C5 & 52 & -5 & 44 & 72 & ; * \\
\hline C\#4 & 554.365 & $539-570$ & $476-5$ & 73 & ; * \\
\hline D5 & 587.33 & $571-6 \odot 4$ & $508-541$ & 74 & $;^{*}$ \\
\hline D\#4 & & $5-640$ & $542-577$ & 75 & \\
\hline E5 & & $1-6$ & & 76 & 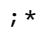 \\
\hline F5 & & -719 & 61 & 77 & \\
\hline F\#5 & $73 s$ & $720-761$ & $657-$ & 78 & ; * \\
\hline G5 & 783.991 & $762-807$ & $699-744$ & 79 & $;^{*}$ \\
\hline G\#5 & 830.609 & $808-855$ & $745-792$ & 80 & \\
\hline A5 & 880 & $856-906$ & $793-8$ & 81 & \\
\hline A\#5 & 932 & $907-960$ & $844-897$ & 82 & \\
\hline B5 & 987.767 & $961-1017$ & $898-954$ & 83 & $i^{*}$ \\
\hline C6 & 1046.502 & $1018-1078$ & $955-1015$ & 84 & \\
\hline
\end{tabular}

$\begin{array}{lll}\text { MIDILIST } & \text { DC.B } & 36,36,36,36,37,37,37,37,38,38,38,38,39,39,39,39 \\ & \text { DC.B } & 39,40,40,40,40,41,41,41,41,41,42,42,42,42,42,42 \\ \text { DC.B } & 43,43,43,43,43,44,44,44,44,44,44,45,45,45,45,45 \\ \text { DC.B } & 45,46,46,46,46,46,46,46,47,47,47,47,47,47,47,48 \\ \text { DC.B } & 48,48,48,48,48,48,49,49,49,49,49,49,49,49,50,50 \\ \text { DC.B } & 50,50,50,50,50,50,50,51,51,51,51,51,51,51,51,51 \\ \text { DC.B } & 52,52,52,52,52,52,52,52,53,53,53,53,53,53,53,53 \\ \text { DC.B } & 53,53,54,54,54,54,54,54,54,54,54,54,54,55,55,55 \\ \text { DC.B } & 55,55,55,55,55,55,55,55,56,56,56,56,56,56,56,56 \\ \text { DC.B } & 56,56,57,57,57,57,57,57,57,57,57,57,57,57,57,58 \\ \text { DC.B } & 58,58,58,58,58,58,58,58,58,58,58,58,58,59,59,59 \\ \text { DC.B } & 59,59,59,59,59,59,59,59,59,59,59,60,60,60,60,60\end{array}$


DC. B

DC. B

DC. B

DC. B

DC. B

DC. B

DC. B

DC. B

DC. B

DC. B

DC. B

DC. B

DC. B

DC. B

DC. B

DC. B

DC. B

DC. B

DC. B

DC. B

DC. B

DC. B

DC. B

DC. B

DC. B

DC. B

DC. B

DC. B

DC. B

DC. B

DC. B

DC. B

DC. B

DC. B

DC. B

DC. B

DC. B

DC. B

DC. B

DC. B

DC. B

DC. B

DC. B

DC. B

DC. B

DC. B

DC. B

DC. B

DC. B

DC. B

DC. B
$6 \odot, 6 \odot, 6 \odot, 6 \odot, 6 \odot, 6 \odot, 6 \odot, 6 \odot, 6 \odot, 61,61,61,61,61,61,61$ $61,61,61,61,61,61,61,61,61,62,62,62,62,62,62,62$ $62,62,62,62,62,62,62,62,62,62,63,63,63,63,63,63$ $63,63,63,63,63,63,63,63,63,63,63,64,64,64,64,64$

$64,64,64,64,64,64,64,64,64,64,64,64,64,64,65,65$ $65,65,65,65,65,65,65,65,65,65,65,65,65,65,65,65$ $65,66,66,66,66,66,66,66,66,66,66,66,66,66,66,66$ $66,66,66,66,66,66,67,67,67,67,67,67,67,67,67,67$ $67,67,67,67,67,67,67,67,67,67,67,67,67,68,68,68$ $68,68,68,68,68,68,68,68,68,68,68,68,68,68,68,68$ $68,68,68,68,69,69,69,69,69,69,69,69,69,69,69,69$ $69,69,69,69,69,69,69,69,69,69,69,69,69,69,70,70$ $70,70,70,70,70,70,70,70,70,70,70,70,70,70,70,70$ $70,70,70,70,70,70,70,70,71,71,71,71,71,71,71,71$ $71,71,71,71,71,71,71,71,71,71,71,71,71,71,71,71$ $71,71,71,72,72,72,72,72,72,72,72,72,72,72,72,72$ $72,72,72,72,72,72,72,72,72,72,72,72,72,72,72,72$ $72,73,73,73,73,73,73,73,73,73,73,73,73,73,73,73$ $73,73,73,73,73,73,73,73,73,73,73,73,73,73,73,73$ $74,74,74,74,74,74,74,74,74,74,74,74,74,74,74,74$

$74,74,74,74,74,74,74,74,74,74,74,74,74,74,74,74$ $74,75,75,75,75,75,75,75,75,75,75,75,75,75,75,75$ $75,75,75,75,75,75,75,75,75,75,75,75,75,75,75,75$ $75,75,75,75,75,76,76,76,76,76,76,76,76,76,76,76$ $76,76,76,76,76,76,76,76,76,76,76,76,76,76,76,76$ $76,76,76,76,76,76,76,76,76,76,77,77,77,77,77,77$ $77,77,77,77,77,77,77,77,77,77,77,77,77,77,77,77$ $77,77,77,77,77,77,77,77,77,77,77,77,77,77,77,77$ $77,77,78,78,78,78,78,78,78,78,78,78,78,78,78,78$ $78,78,78,78,78,78,78,78,78,78,78,78,78,78,78,78$ $78,78,78,78,78,78,78,78,78,78,78,79,79,79,79,79$ $79,79,79,79,79,79,79,79,79,79,79,79,79,79,79,79$ $79,79,79,79,79,79,79,79,79,79,79,79,79,79,79,79$ $79,79,79,79,79,79,79,79,80,80,80,80,80,80,80,80$ $80,80,80,80,80,80,80,80,80,80,80,80,80,80,80,80$ $80,80,80,80,80,80,80,80,80,80,80,80,80,80,80,80$

$80,80,80,80,80,80,80,81,81,81,81,81,81,81,81,81$ $81,81,81,81,81,81,81,81,81,81,81,81,81,81,81,81$ $81,81,81,81,81,81,81,81,81,81,81,81,81,81,81,81$ $81,81,81,81,81,81,81,81,81,82,82,82,82,82,82,82$ $82,82,82,82,82,82,82,82,82,82,82,82,82,82,82,82$ $82,82,82,82,82,82,82,82,82,82,82,82,82,82,82,82$ $82,82,82,82,82,82,82,82,82,82,82,82,82,82,83,83$ $83,83,83,83,83,83,83,83,83,83,83,83,83,83,83,83$ $83,83,83,83,83,83,83,83,83,83,83,83,83,83,83,83$ $83,83,83,83,83,83,83,83,83,83,83,83,83,83,83,83$ $83,83,83,83,83,83,84,84,84,84,84,84,84,84,84,84$ $84,84,84,84,84,84,84,84,84,84,84,84,84,84,84,84$ $84,84,84,84,84,84,84,84,84,84,84,84,84,84,84,84$ $84,84,84,84,84,84,84,84,84,84,84,84,84,84,84,84$

84,84 